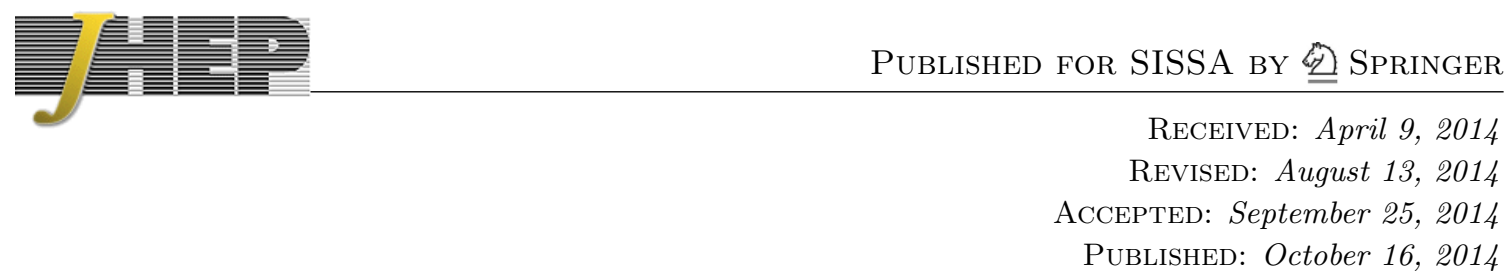

\title{
The non-linear evolution of jet quenching
}

\author{
Edmond lancu \\ Institut de Physique Théorique de Saclay, \\ F-91191 Gif-sur-Yvette, France \\ E-mail: edmond.iancu@cea.fr
}

ABSTRACT: We construct a generalization of the JIMWLK Hamiltonian, going beyond the eikonal approximation, which governs the high-energy evolution of the scattering between a dilute projectile and a dense target with an arbitrary longitudinal extent (a nucleus, or a slice of quark-gluon plasma). Different physical regimes refer to the ratio $L / \tau$ between the longitudinal size $L$ of the target and the lifetime $\tau$ of the gluon fluctuations. When $L / \tau \ll 1$, meaning that the target can be effectively treated as a shockwave, we recover the JIMWLK Hamiltonian, as expected. When $L / \tau \gg 1$, meaning that the fluctuations live inside the target, the new Hamiltonian governs phenomena like transverse momentum broadening and radiative energy loss, which accompany the propagation of an energetic parton through a dense QCD medium. Using this Hamiltonian, we derive a non-linear equation for the dipole amplitude (a generalization of the BK equation), which describes the high-energy evolution of jet quenching. As compared to the original BK-JIMWLK evolution, the new evolution is remarkably different: the plasma saturation momentum evolves much faster with increasing energy (or decreasing Bjorken's $x$ ) than the corresponding scale for a shockwave. This widely opens the transverse phase-space for the evolution in the vicinity of the saturation line and implies the existence of large radiative corrections, enhanced by the double logarithm $\ln ^{2}(L T)$, with $T$ the temperature of the medium. This confirms from a wider perspective a recent result by Liou, Mueller, and Wu (arXiv:1304.7677). The dominant, double-logarithmic, corrections to the dipole amplitude are smooth enough to be absorbed into a renormalization of the jet quenching parameter $\hat{q}$. This renormalization is universal: it applies to all the phenomena, like the transverse momentum broadening or the radiative energy loss, which can be computed from the dipole amplitude.

KeYwords: Resummation, Renormalization Group, QCD, Quark-Gluon Plasma

ARXIV EPRINT: 1403.1996

Open AcCess, (c) The Authors.

Article funded by $\mathrm{SCOAP}^{3}$.

doi:10.1007/JHEP10(2014)095 


\section{Contents}

1 Introduction 1

2 The evolution Hamiltonian in the high-energy approximation $\quad 7$

2.1 The evolution Hamiltonian 8

$\begin{array}{lll}2.2 & \text { Virtual corrections and probability conservation } & 12\end{array}$

3 A shockwave target: recovering the JIMWLK Hamiltonian 15

$\begin{array}{ll}3.1 \text { Performing the time integrations } & 16\end{array}$

$\begin{array}{ll}3.2 & \text { The Balitsky-Kovchegov equation } \\ & 20\end{array}$

4 The high-energy evolution of transverse momentum broadening $\quad 24$

$\begin{array}{ll}4.1 \text { The tree-level approximation } & 25\end{array}$

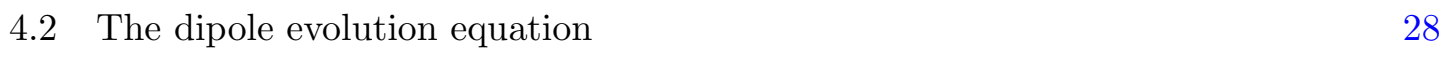

4.3 The single scattering approximation 35

4.3.1 The BFKL equation for jet quenching 35

$\begin{array}{lll}\text { 4.3.2 The double logarithmic approximation } & 39\end{array}$

4.3.3 The phase-space for the high-energy evolution 43

4.4 Gluon evolution and saturation in the medium 46

4.5 Comments on the effects of multiple scattering 50

5 The high-energy evolution of the radiative energy loss 53

5.1 The tree-level approximation: the BDMPSZ formalism 53

$\begin{array}{ll}5.2 & \text { The dominant radiative corrections } \\ \end{array}$

6 Conclusions and perspectives $\quad 62$

$\begin{array}{ll}\text { A A succinct derivation of the evolution Hamiltonian } & 64\end{array}$

$\begin{array}{lr}\text { B The background field gluon propagator } & 68\end{array}$

$\begin{array}{lll}\text { C A sum rule for the light-cone gauge propagator } & 70\end{array}$

$\begin{array}{ll}\text { D Finite- } N_{c} \text { corrections within the mean field approximation } & 70\end{array}$

\section{Introduction}

The concept of jet quenching globally denotes the modifications in the properties of a 'hard probe' (an energetic parton, or the jet generated by its evolution) which occur when this 'jet' propagates through the dense QCD matter ('quark-gluon plasma') created in the intermediate stages of a ultrarelativistic nucleus-nucleus collision [1-5]. This encompasses 
several related phenomena like the transverse momentum broadening, the (radiative) energy loss, or the jet fragmentation via medium-induced gluon branching, and also the associated observables, like the nuclear modification factor, or the di-jet asymmetry. A common denominator of these phenomena is that, within most of their theoretical descriptions to date, they depend upon the medium properties via a single parameter: a transport coefficient known as the 'jet quenching parameter' $\hat{q}$. This explains the importance of this quantity $\hat{q}$ for both theory and phenomenology, and motivates the recent attempts to obtain better estimates for it from first principles, at least in special cases [6-11].

Roughly speaking, the jet quenching parameter measures the dispersion in transverse momentum accumulated by a fast parton after crossing the medium over a distance $L$ : $\left\langle p_{\perp}^{2}\right\rangle \simeq \hat{q} L$. At weak coupling, the dominant mechanism responsible for this dispersion is multiple scattering off the medium constituents. At leading order in $\alpha_{s}, \hat{q}$ can be computed as the second moment of the 'collision kernel' (see section 4.1 for details). Beyond leading order, one needs a non-perturbative definition for $\left\langle p_{\perp}^{2}\right\rangle$. The one that we shall adopt here and which is often used in the literature involves the 'color dipole', a light-like Wilson loop in the color representation of the fast parton. Physically, this Wilson loop describes the $S$-matrix $\mathcal{S}(r)$ for a small 'color dipole' (say, a quark-antiquark pair in a color singlet state) with transverse size $r$ which propagates through the medium. Via unitarity, the Fourier transform of $\mathcal{S}(r)$ determines the transverse-momentum distribution $\mathrm{d} N / \mathrm{d}^{2} \boldsymbol{p}$ of the parton when it exits the medium [12, 13]. At tree-level, these definitions imply $\left\langle p_{\perp}^{2}\right\rangle^{(0)} \simeq \hat{q}^{(0)}(L) L$, with $\hat{q}^{(0)}(L)$ logarithmically dependent upon the medium size $L$. This dependence enters via the resolution of the scattering process: the transverse momenta transferred by the collision can be as large as the 'saturation momentum' $Q_{s}^{2}(L) \equiv \hat{q} L$. Beyond leading order, it is a priori unclear whether the notion of 'jet quenching parameter' (as a quasi-local transport coefficient) is still useful, or even well-defined. Our criterion in that sense will be to check whether a formula like $\left\langle p_{\perp}^{2}\right\rangle \simeq \hat{q}(L) L$ does still hold, with $\hat{q}(L)$ a reasonably smooth function.

This criterion appears to be satisfied for the two classes of next-to-leading order corrections to $\left\langle p_{\perp}^{2}\right\rangle \simeq \hat{q} L$ that have been considered so far and which correspond to very different kinematical regimes [7,9]. For a weakly-coupled quark-gluon plasma (QGP) with temperature $T$, Caron-Huot has computed the corrections of $\mathcal{O}(g)$ to the 'collision kernel', as generated by the soft, highly-populated, thermal modes, with energies and momenta $\lesssim g T$ [7]. (The corresponding leading-order value has been computed by Arnold and Xiao [6].) These corrections do not modify the logarithmic dependence of $\hat{q}$ upon the medium size $L$, which is rather introduced by the hardest collisions, with transferred momenta $k_{\perp} \sim Q_{s}$. (Throughout this paper, we assume that $Q_{s}(L) \gg T$.)

By contrast, in ref. [9], Liou, Mueller, and Wu have studied the relatively hard and nearly on-shell gluon fluctuations, with large transverse momenta $p_{\perp} \gg T$ and even larger longitudinal momenta $p^{3} \simeq p^{0} \gg p_{\perp}$ (in the plasma rest frame). Such fluctuations, which are most naturally viewed as bremsstrahlung by the projectile, are not sensitive to the detailed properties of the medium. They depend upon the latter only via the tree-level value $\hat{q}^{(0)}$ of the jet quenching parameter and via two basic scales - the longitudinal size $L$ and the wavelength $\lambda$ of the typical medium constituents (with $\lambda=1 / T$ for the 
QGP) — which constrain the phase-space for bremsstrahlung. Ref. [9] found large oneloop corrections ${ }^{1}$ to $\left\langle p_{\perp}^{2}\right\rangle$, of relative order $\alpha_{s} N_{c} \ln ^{2}(L / \lambda)$, where the double logarithm comes from the phase-space: one logarithm is generated by integrating over the lifetime $\tau \sim p^{0} / p_{\perp}^{2}$ of the fluctuations, over the range $\lambda \ll \tau \ll L$, and the other one comes from the respective transverse momenta, within the interval $\hat{q} \tau \ll p_{\perp}^{2} \ll Q_{s}^{2}(L)$. The lower limit $\hat{q} \tau$ on $p_{\perp}^{2}$ refers to multiple scattering: the condition $p_{\perp}^{2} \gg \hat{q} \tau$ means that the relevant fluctuations are hard enough to suffer only one scattering during their lifetime. Physically, these radiative corrections express the contribution to the transverse momentum broadening coming from the recoil associated with unresolved emissions.

It is important to notice that the medium size $L$ sets the upper limit on the lifetime $\tau$ of the fluctuations, hence on their energy $p^{0}$. Accordingly, when increasing $L$, one opens the phase-space for fluctuations which are more and more energetic. Such fluctuations can then evolve towards lower energies, via soft gluon emissions. This evolution is represented by Feynman graphs of higher-loop order (gluon cascades which are strongly ordered in energy), which are enhanced by the phase-space: the powers of $\bar{\alpha} \equiv \alpha_{s} N_{c} / \pi$ associated with soft gluon emissions can be accompanied by either double, or at least single, logarithms of $L / \lambda$, depending upon the kinematics of the emissions. Ref. [9] not only computed the first step in this evolution, for both the double-logarithmic and the single-logarithmic corrections, but also provided a simple recipe for resuming the corrections enhanced by double-logarithms to all orders. This resummation is tantamount to a renormalization of the jet quenching parameter, although this interpretation has not been explicitly spelled out in [9]. In fact, the suggestion that the double logarithmic correction calculated in [9] could be interpreted as a renormalization of $\hat{q}$, with a universal character, was first made by Mehtar-Tani [15] and later developed (in the special context of the radiative energy loss) by Blaizot and Mehtar-Tani [16].

Yet, already the one-loop calculation of the single-logarithmic corrections in ref. [9] has met with several difficulties, reflecting the lack of a systematic theoretical framework for this complicated, non-linear, evolution. Namely, in order to compute the effects of order $\bar{\alpha} \ln (L / \lambda)$, one had to estimate the effects of multiple scattering beyond the eikonal approximation and also to heuristically include the 'virtual' corrections responsible for probability conservation, that were otherwise missed by that analysis. Vice-versa, the only reason why the double-logarithmic corrections appear to be comparatively simple, is because they are neither sensitive to multiple scattering (except for the restriction on their phase-space), nor to the effects of the 'virtual' corrections.

We thus see that the subset of radiative corrections which are enhanced by powers of $\ln ^{2}(L / \lambda)$ play a special role in the in-medium evolution of dipole $S$-matrix: they form an 'island' of effectively linear evolution (so they are relatively easy to compute), they dominate in the limit of a large medium $L \gg \lambda$, and they are smooth enough to be absorbed into a redefinition of $\hat{q}$. Such considerations demonstrate the importance of the double-logarithmic approximation (DLA) for the high-energy evolution of jet quenching.

\footnotetext{
${ }^{1}$ See also ref. [14] for a similar but earlier observation, which has motivated the more elaborate analysis in ref. [9].
} 
It will be one of our main objectives in this paper to understand the emergence of this approximation from a more general perspective and with a clear physical interpretation. This is indeed non-trivial, as we now explain.

By itself, the prominence of DLA within the pQCD evolution is not new - it is a rather generic consequence of the soft and collinear divergences of the bremsstrahlung. Other familiar examples include the fragmentation of a virtual jet in the vacuum [17], or the evolution of the gluon distribution in the 'double-leading-log-approximation' (a common limit of the DGLAP and BFKL equations [18]). What is surprising though, is the importance of such an approximation in the context of a non-linear evolution. All the other examples listed above refer to linear processes. And in the only other example of a non-linear pQCD evolution at our disposal - the BK-JIMWLK evolution of the gluon distribution in a large nucleus (or of particle production in proton-nucleus collisions) [19$29]$ - it is well known that the DLA becomes a good approximation only in the linear regime at very large transverse momenta $k_{\perp} \gg Q_{s}$, or very small dipole sizes $r \ll 1 / Q_{s}$. But this is not the most interesting regime for a study of jet quenching. Indeed, as we shall later argue, phenomena like $p_{\perp}$-broadening and the radiative energy loss are controlled by dipole sizes in the vicinity of the saturation line: $r \lesssim 1 / Q_{s}$. The evolution in this regime turns out to be very different in the context of jet quenching as compared to $p A$ collisions.

To better appreciate the differences, let us first remind that, for a nuclear target, the high-energy evolution of the dipole $S$-matrix is governed by the non-linear BK equation [19, 20]. Both the non-linear effects in this equation (corresponding to multiple scattering and gluon saturation in the target) and the 'virtual' corrections (for probability conservation) are important in the approach towards saturation. Together, they imply a drastic change in the behavior of the scattering amplitude $\mathcal{T}(r) \equiv 1-\mathcal{S}(r)$ for $r \lesssim 1 / Q_{s}$ : the corresponding result at tree-level, $\mathcal{T}^{(0)}(r) \sim r^{2} \ln r^{2}$, gets replaced via the evolution by $\mathcal{T}(r) \sim r^{2 \gamma_{s}}$ with $\gamma_{s} \simeq 0.63$ a non-perturbative anomalous dimension [30-33]. If a similar change of behavior was to occur also in the problem of jet quenching, it could not be simply absorbed into a redefinition of $\hat{q}$. Vice-versa, if the dominant radiative corrections to jet quenching appear to be consistent with a mere renormalization of $\hat{q}[9,15,16]$, it is essentially because, in this particular context, the DLA remains valid in the vicinity of the saturation line. Indeed, as we shall demonstrate in section 4.3.2, the DLA evolution preserves the same functional form for $\mathcal{S}(r)$ as at tree-level, except for the replacement of the tree-level jet quenching parameter $\hat{q}^{(0)}$ by its renormalized value $\hat{q}(L)$, which obeys eq. (4.42). In turn, this implies the universality of the renormalization of the jet quenching parameter, to DLA accuracy: all the quantities that can be computed from the dipole $S$-matrix get renormalized simply via the appropriate redefinition of $\hat{q}$.

We are now in a position to describe the new developments in this paper. To demonstrate the key ideas alluded to above, like the emergence of the DLA in the approach towards saturation, or the universality of the renormalization of $\hat{q}$, and also to be able to go beyond DLA (at least in principle), one needs a theory for the non-linear evolution of jet quenching. That is, one needs equations describing the evolution of observables like the dipole $S$-matrix under a change in the medium size $L$, within the high-energy approximations. In what follows, we shall develop such a theory via a suitable generalization of 
the BK-JIMWLK evolution. Like the latter, the new theory is valid to leading logarithmic accuracy, meaning that it resums terms of order $(\bar{\alpha} \ln L / \lambda)^{n}$ for any $n \geq 1$ together with the non-linear effects associated with multiple scattering and gluon saturation in the medium. However, and unlike for the BK-JIMWLK evolution, the logarithmic enhancement for the in-medium evolution is not easy to demonstrate in full generality, because of the non-local structure of the multiple scattering in time (equivalently, because of the failure of the eikonal approximation; see below). This being said, we shall be able to demonstrate this enhancement in the single scattering approximation (see section 4.3) and also for multiple scattering under specific approximations (see section 4.5).

In developing the formalism below, it will be convenient to assume that the projectile enters the medium from the outside and that it was on-shell prior to the collision. This guarantees that the quantum fluctuations which matter for the evolution of the $S$-matrix are generated exclusively via interactions in the target. ${ }^{2}$ Then the main difference between the evolution of jet quenching and that of $p A$ collisions refers to the ratio between the longitudinal extent $L$ of the target and the lifetime $\tau$ of the gluon fluctuations. In $p A$ collisions, the center-of-mass energy is so high that the nuclear target looks effectively like a shockwave $(L \ll \tau)$, due to Lorentz contraction. Then the multiple scattering can be treated in the strict eikonal approximation, which assumes that the transverse coordinates of the projectile partons are not affected by their interactions in the medium. By contrast, in the context of jet quenching, the energies are much lower and the typical fluctuations live inside the medium $(L \gtrsim \tau)$, so the effects of the multiple scattering can accumulate during their whole lifetime. Then the strict eikonal approximation is not applicable anymore, although the individual scatterings are still soft: one cannot ignore the transverse motion of the fluctuations during their lifetime.

These considerations also show that these two problems, $p A$ collisions and jet quenching, can be viewed as limiting situations of a common set-up: the high-energy scattering between a dilute projectile and a dense target with an arbitrary longitudinal extent. This is the first problem that we shall address and solve in this paper. Specifically, in section 2 and appendix A, we shall construct an effective Hamiltonian which, when acting on the $S$-matrix of the projectile (a gauge-invariant product of Wilson lines), generates one additional soft gluon emission in the background of a strong color field representing the medium. (The medium correlations are reproduced by averaging over this background field, in the spirit of the color glass condensate $[35,36]$.) This Hamiltonian provides a generalization of the JIMWLK Hamiltonian beyond the eikonal approximation. It looks compact and simple, but it is less explicit than the JIMWLK Hamiltonian, in the sense that the integrals over the emission times cannot be performed in general (i.e. for an arbitrary target). Accordingly, the general Hamiltonian is non-local both in the transverse coordinates and in the light-cone (LC) times. The formal manipulations with this Hamiltonian are complicated by potential (infrared and ultraviolet) divergences which require prescriptions at the

\footnotetext{
${ }^{2}$ If the projectile is produced by a hard process occurring inside the medium or at some finite distance from it, then there is additional radiation, associated with the initial virtuality, that would mix with the evolution that we are here interested in (see e.g. the discussion in [34]). By choosing an on-shell projectile, we avoid this mixing.
} 
intermediate steps and cancel only in the final results. In section 2.2 , we demonstrate a general mechanism ensuring such cancellations - this involves a particular 'sum-rule' for the gluon propagator in the LC gauge, eq. (2.13) - and clarify its connexion to probability conservation. In particular, we show that the 'virtual' corrections can be alternatively implemented as a local 'counter-term', which is particularly convenient when the target is an extended medium.

As a first test of the new Hamiltonian and of our ability to use it in practice, we consider in section 3 the example of a shockwave target $(L \ll \tau)$. In that case, the integrals over the emission times can be explicitly performed (the adiabatic prescription for regulating the large time behavior turns out to be important for that purpose) and, as a result, we recover the JIMWLK Hamiltonian [21-29], as expected. We also show that the 'counterterm' alluded to above generates the 'virtual' piece in the BK equation - once again, as expected.

Starting with section 4 , we turn to the case of an extended target $(L \gg \tau)$, as appropriate for the problem of jet quenching. The general equations generated by the evolution Hamiltonian in that case are extremely complicated (see section 4.2): they are non-local in $L C$ time (since gluon emissions can occur anywhere inside the medium and they can have any lifetime $\tau$ ) and also functional (the transverse trajectories of the gluon fluctuations are random, due to quantum diffusion, and distributed according to a path-integral). An useful approximation is to assume that the medium correlations are Gaussian and local in LC time. (A similar mean field approximation has proven to be successful in the case of the BK-JIMWLK equations [35, 37-44].) Under this assumption, the equation obeyed by the dipole $S$-matrix takes the form shown in eqs. (4.22) or (4.24), which is recognized as a functional generalization of the BK equation. It remains as an open question whether such an equation can be solved via numerical methods.

Our main point though is that, for the present purposes - i.e. for a study of the leading-order evolution of the jet quenching in the limit $L \gg \lambda-$, one can drastically simplify this equation and even obtain analytic results. This is so because the dominant radiative corrections are those enhanced by the double logarithm $\ln ^{2}(L / \lambda)[9]$ and they are encoded into a much simpler, linear, version of eq. (4.24), which corresponds to a single scattering approximation (see section 4.3). A subtle point in this context is the fact that the relevant linear approximation is not the BFKL equation (4.29), as it would be for a shockwave, but rather its simpler, double-logarithmic, version in eq. (4.33). This follows from a study of the phase-space for the linear evolution, as constrained by the non-linear effects in eq. (4.24). As anticipated, the DLA preserves the tree-level functional form of the dipole $S$-matrix and thus can be written as an equation for the renormalized jet quenching parameter $\hat{q}(L)$, namely eq. (4.42). This is equivalent with the resummation performed in [9] in the context of $p_{\perp}$-broadening and also with the equation inferred from Feynman graphs in $[15,16]$ in the context of radiative energy loss. Eq. (4.42) differs from the standard DLA equation in the literature [18] via the integration limits, which here reflect the non-linear physics of multiple scattering. The solution $\hat{q}(L)$ to this equation shows a stronger dependence upon the medium size $L$ than at tree-level, due to the non-locality of the radiative corrections.

Given the central role played here by the DLA and the difference in that respect with the case of a shockwave, it is interesting to understand the emergence of this approximation 
on more physical grounds. As we explain in section 4.4, this is related to the special way how gluon saturation occurs in a medium: the non-linear effects in the evolution of the dipole amplitude can be also understood as saturation effects in the gluon distribution in the medium, but with a saturation scale $Q_{s}^{2}(x)$ which increases very fast with $1 / x-$ much faster than the corresponding scale for a shockwave. $(x=\lambda / \tau$ is the longitudinal momentum fraction of the gluons.) Specifically, one finds $Q_{s}^{2}(x)=\hat{q} \lambda / x$, which rises as $1 / x$ already at tree-level. The physical explanation is quite simple: the quantity $Q_{s}^{2}(x)$ is proportional with the longitudinal size of the region where the gluons can overlap with each other; for gluons inside the medium, this region is their wavelength $\tau=\lambda / x$. This rapid rise of $Q_{s}^{2}(x)$ with $1 / x$, which becomes even more pronounced after taking into account the small- $x$ evolution of $\hat{q}$ (see eq. (4.63)), implies that the transverse phase-space for the high-energy evolution towards saturation grows as fast as the longitudinal one. In turn, this creates the conditions for a double-logarithmic evolution. ${ }^{3}$ By contrast, in the case of a shockwave, the $x$-dependence of $Q_{s}^{2}(x)$ is perturbatively small (since a consequence of the evolution), so the transverse phase-space increases much slower than the longitudinal one in the approach towards saturation.

As a final application, we consider in section 5 the evolution of the radiative energy loss, within the framework of the BDMPSZ mechanism for medium-induced gluon radiation [4656]. This is essentially a variation of the problem discussed in section 4 , in the sense that the BDMPSZ spectrum is itself related to the dipole $S$-matrix. The only new feature is that, now, the eikonal approximation fails not only for the soft gluon fluctuation responsible for the evolution, but also for its relatively hard parent gluon, which is responsible for the energy loss. That is, one has to study the evolution of a non-eikonal dipole. Yet, this brings no serious difficulty because of the strong separation in energy, hence in lifetime, between the fluctuations and the radiation. In particular, at DLA, the evolution of the radiative energy loss is obtained by simply using the renormalized value of $\hat{q}$ (the solution to eq. (4.42)) within the respective formula at tree-level, in agreement with refs. $[15,16]$.

Section 6 briefly summarizes our results and conclusions and lists some open problems.

\section{The evolution Hamiltonian in the high-energy approximation}

Throughout this paper, we shall consider the high-energy evolution of the scattering amplitude for the collision between a dilute projectile and a dense target. The projectile is a set of partons in an overall color singlet state (the prototype being a color dipole), while the target can be either a large nucleus, or the dense partonic medium created in the intermediate stage of an ultrarelativistic heavy ion collision. In both cases, the target is characterized by a dense gluon distribution, which for the present purposes will be described in the spirit of the CGC formalism, that is, as a random distribution of strong, classical, color fields. The interactions between the projectile and the target will be treated in a generalized eikonal approximation, which allows one to resum the multiple scattering

\footnotetext{
${ }^{3} \mathrm{~A}$ double-logarithmic evolution for gluons in the plasma has also been advocated in ref. [45]. In that case though, this was the standard DLA limit of either BFKL or DGLAP equation, which applies only for $k_{\perp} \gg Q_{s}$.
} 
between the partons in the projectile and the strong color fields in the target to all orders, via Wilson lines, while also keeping trace of the transverse motion of the partons.

One step in the high-energy evolution consists in the emission of a relatively soft gluon by one of the partons in the projectile and in the background of the target field. Such an emission modifies the partonic content of the projectile and hence the $S$-matrix for the elastic scattering between the projectile and the target. In this section we shall present and motivate a rather compact expression for the Hamiltonian which 'generates this evolution', that is, which describes the change in the $S$-matrix induced by one soft gluon emission. A schematic derivation of this Hamiltonian from the QCD path integral, which largely follows the derivation of the JIMWLK Hamiltonian in [26, 29], will be presented in appendix A.

\subsection{The evolution Hamiltonian}

To be specific, let us assume that the projectile propagates in the positive $x^{3}$ direction and introduce light-cone (LC) vector notations: $x^{\mu}=\left(x^{+}, x^{-}, \boldsymbol{x}\right)$, with $x^{+}=\left(x^{0}+x^{3}\right) / \sqrt{2}$, $x^{-}=\left(x^{0}-x^{3}\right) / \sqrt{2}$, and $\boldsymbol{x}=\left(x^{1}, x^{2}\right)$. Each parton in the projectile has a color current oriented in the LC 'plus' direction, which couples to the $A_{a}^{-}$component of the target color field. If the parton energy is sufficiently high (see below for the precise condition), its transverse coordinate $\boldsymbol{x}$ is not affected by the interaction. Then the only effect of the latter is a rotation of the parton color state, as encoded in the Wilson line:

$$
U^{\dagger}(\boldsymbol{x})=\mathrm{P} \exp \left\{\mathrm{i} g \int \mathrm{d} x^{+} A_{a}^{-}\left(x^{+}, \boldsymbol{x}\right) T^{a}\right\} .
$$

The $T^{a}$ 's are the color group generators in the appropriate representation and $\mathrm{P}$ stands for path ordering w.r.t. $x^{+}$(the LC 'time' for the projectile): with increasing $x^{+}$, matrices are ordered from right to left. The integral over $x^{+}$formally extends along the whole real axis, but in practice it is limited to the support of the target field. The $x^{-}$coordinate has been omitted in eq. (2.1) since it is understood that $x^{-} \simeq 0$ for the ultrarelativistic projectile, by Lorentz contraction.

The elastic $S$-matrix for a color-singlet projectile involves the trace of a product of such Wilson lines, one for each parton (quark, antiquark, or gluon) in the projectile. For more clarity, in what follows we shall keep the notations $T^{a}$ and $U^{\dagger}$ for the color group generators and the Wilson lines in the adjoint representation, and use $t^{a}$ and respectively $V^{\dagger}$ for quarks in the fundamental representation. As anticipated, most of the examples below will refer to a color dipole, for which the $S$-matrix reads (in the fundamental representation, for definiteness)

$$
\hat{S}_{\boldsymbol{x} \boldsymbol{y}} \equiv \frac{1}{N_{c}} \operatorname{tr}\left[V_{\boldsymbol{x}}^{\dagger} V_{\boldsymbol{y}}\right]
$$

where $\boldsymbol{x}$ and $\boldsymbol{y}$ are the transverse coordinates of the quark and the antiquark, respectively, and $V_{\boldsymbol{x}}^{\dagger} \equiv V^{\dagger}(\boldsymbol{x})$, etc. This dipole enters the calculation of a variety of physical processes, like the total cross-section for deep inelastic scattering, the cross-section for single inclusive hadron production in proton-nucleus $(p A)$ collisions, or the transverse momentum broadening of a 'hard probe' (here an energetic quark) propagating through the dense partonic 
medium ('quark-gluon plasma') created at the intermediate stages of a nucleus-nucleus $(A A)$ collision.

Below we shall refer to eq. (2.1) as the strict eikonal approximation. For a quantum particle, like the gluon fluctuations that we are interested in, this is correct only so long as the target is 'sufficiently thin' - namely, so long as the duration $\Delta x^{+}$of the interaction is small enough for the effects of the quantum diffusion to remain negligible. Indeed, a high energy particle with longitudinal momentum $p^{+}$is similar to a non-relativistic quantum particle with mass equal to $p^{+}$and living in two spatial dimensions, in that it undergoes Brownian motion in the transverse plane: the dispersion $\Delta x_{\perp}^{2}$ in its transverse position grows with time according to $\Delta x_{\perp}^{2} \simeq \Delta x^{+} / 2 p^{+}$. (This transverse dynamics is explicit in eq. (2.9) below.) The dispersion thus accumulated during the interaction time $\Delta x^{+}$can be neglected so long as it remains smaller than the respective quantum uncertainty $1 / p_{\perp}^{2}$ (with $p_{\perp}=|\boldsymbol{p}|$ the particle transverse momentum). This requires ${ }^{4} \Delta x^{+} \ll \tau_{\text {coh }} \equiv 2 p^{+} / p_{\perp}^{2}$, a condition which is well satisfied when the target is a shockwave, but not also in the case of an extended medium.

Indeed, the case of a 'shockwave target' corresponds, by definition, to a physical situation where the collision energy in the center-of-mass frame is so high that the longitudinal extent of the target (as measured in a given Lorentz frame) is much smaller than the coherence time of the relevant partons from the projectile in that particular frame. In particular, if the scattering is viewed in a frame where the target is highly boosted, then the target looks genuinely as a shockwave. But the statement about the ratio between the target width and the coherence time of the partons in the projectile is of course boost invariant: in the shockwave set-up, this ratio is small in any frame. This particular set-up corresponds e.g. to proton-nucleus $(p A)$ collisions at the LHC energies.

By contrast, the target looks like an 'extended medium' when the center-of-mass energy is not that high and the coherence times of the relevant partons from the projectile become comparable to, or even smaller than, the longitudinal width $L$ of the target. In what follows, we shall often be interested in such (relatively short-lived) parton fluctuations, with $\tau_{c o h}=2 p^{+} / p_{\perp}^{2} \lesssim L$. In such a case, the duration $\Delta x^{+}$of the interaction process is equal to $\tau_{c o h}$ and the effects of the transverse diffusion are generally important. To take that into account, we need the generalization of eq. (2.1) to an arbitrary trajectory $\boldsymbol{x}(t)$ in the transverse plane $\left(t \equiv x^{+}\right.$is the LC time). This is a functional of the trajectory, which reads

$$
U_{t_{2} t_{1}}^{\dagger}[\boldsymbol{x}(t)]=\mathrm{P} \exp \left\{\mathrm{i} g \int_{t_{1}}^{t_{2}} \mathrm{~d} t A_{a}^{-}(t, \boldsymbol{x}(t)) T^{a}\right\} .
$$

As compared to eq. (2.1) we have also generalized the definition in eq. (2.3) to trajectories which start at some generic (light-cone) time $t_{1}$ and end up at a later time $t_{2}$. In writing eq. (2.3), we have implicitly assumed that the transverse velocity and momentum of the partonic fluctuations are much smaller than the corresponding longitudinal quantities (say, as measured in the rest frame of the medium). Hence, one can ignore the vectorial coupling

\footnotetext{
${ }^{4}$ In evaluating the coherence time $\tau_{c o h}$ one should use the maximal value of $p_{\perp}$ accumulated by the particle via rescattering in the target, that is, the saturation momentum $Q_{s}$ to be later introduced.
} 
to the transverse components of the target gauge field. This condition $p_{\perp} \ll p^{+}$is indeed well satisfied for the problems of interest and, whenever needed, will be enforced as a constraint on the phase-space (see e.g. the discussion in section 4.3.3).

We are now in a position to present the operator which generates the emission of a soft gluon by the dilute projectile in the presence of the strong color field of the target. This operator acts on gauge-invariant operators built with products of Wilson lines, like that in eq. (2.2), and reads

$$
\Delta H=\frac{1}{2} \int \frac{\mathrm{d} p^{+}}{2 \pi} \int \mathrm{d} t_{1} \int \mathrm{d} t_{2} \int \mathrm{d}^{2} \boldsymbol{r}_{2} \int \mathrm{d}^{2} \boldsymbol{r}_{1} J^{a}\left(t_{2}, \boldsymbol{r}_{2}\right) G_{a b}^{--}\left(t_{2}, \boldsymbol{r}_{2} ; t_{1}, \boldsymbol{r}_{1} ; p^{+}\right) J^{b}\left(t_{1}, \boldsymbol{r}_{1}\right),
$$

in notations to be explained below.

The variable $p^{+}$is the LC longitudinal momentum of the emitted gluon; by assumption this is much smaller than the respective momentum of the parent parton (to be below denoted as $\Lambda$ ), but much larger than any 'plus' component that can be transferred by the target in the collision process. Accordingly, the component $p^{+}$is conserved by the interactions, which makes it useful to use the mixed Fourier representation $\left(t, \boldsymbol{x}, p^{+}\right)$, as we did above. The 'strip integral' in eq. (2.4) runs over an interval in $p^{+}$which is symmetric around $p^{+}=0$ :

$$
\int_{\text {strip }} \frac{\mathrm{d} p^{+}}{2 \pi} f\left(p^{+}\right) \equiv\left(\int_{x \Lambda}^{\Lambda}+\int_{-\Lambda}^{-x \Lambda}\right) \frac{\mathrm{d} p^{+}}{2 \pi} f\left(p^{+}\right)=\int_{x \Lambda}^{\Lambda} \frac{\mathrm{d} p^{+}}{2 \pi}\left[f\left(p^{+}\right)+f\left(-p^{+}\right)\right],
$$

Here $\Lambda$ is the typical 'plus' momentum of the emitters, which is the relevant 'hard' scale, whereas $x$, with $x \ll 1$, is the smallest longitudinal fraction of the emitted, 'soft', gluon. In what follows, we shall be mostly concerned with situations where the above integral is logarithmic, $\int\left(\mathrm{d} p^{+} / p^{+}\right)$; in such a case, the evolution operator takes of the form $\Delta H=$ $H_{\text {evol }} \ln (1 / x)$, with $H_{\text {evol }}$ playing the role of a Hamiltonian for the evolution with 'time' $Y \equiv \ln (1 / x)$ (the rapidity difference between the valence partons in the projectile and the softest evolution gluons).

Furthermore, $J^{a}(t, \boldsymbol{r})$ denotes the functional derivative w.r.t. the component $A_{a}^{-}(t, \boldsymbol{r})$ of the gauge field and plays the role of the color charge density operator. When acting on a Wilson line like that in eq. (2.3), this operator generates the emission of a soft gluon from the parton represented by that Wilson line:

$$
\begin{aligned}
J^{a}(t, \boldsymbol{r}) U_{t_{2} t_{1}}^{\dagger}[\boldsymbol{x}] & \equiv \frac{\delta}{\delta A_{a}^{-}(t, \boldsymbol{r})} U_{t_{2} t_{1}}^{\dagger}[\boldsymbol{x}] \\
& =\mathrm{i} g \theta\left(t_{2}-t\right) \theta\left(t-t_{1}\right) \delta^{(2)}(\boldsymbol{r}-\boldsymbol{x}(t)) U_{t_{2} t}^{\dagger}[\boldsymbol{x}] T^{a} U_{t t_{1}}^{\dagger}[\boldsymbol{x}]
\end{aligned}
$$

As visible on this equation, each functional derivative brings a factor of $g$, so $\Delta H$ starts at order $g^{2}=4 \pi \alpha_{s}$ (but in general includes effects of higher order in $g$, via the background field; see below). The operator $J^{a}(t, \boldsymbol{x})$ is also the generator of the infinitesimal color 
rotations. Using (2.6), one can check the following equal-time commutation relation (with $f^{a b c}$ the structure constants for $\mathrm{SU}\left(N_{c}\right)$ and $\left.\delta_{\boldsymbol{x} \boldsymbol{y}} \equiv \delta^{(2)}(\boldsymbol{x}-\boldsymbol{y})\right)$

$$
\left[J^{a}(t, \boldsymbol{x}), J^{b}(t, \boldsymbol{y})\right]=-g \delta_{\boldsymbol{x} \boldsymbol{y}} f^{a b c} J^{c}(t, \boldsymbol{x}),
$$

which confirms that these operators obey the color group algebra, as they should.

The last ingredient in eq. (2.4) is the background field propagator $G^{--}$of the emitted gluon. This is a functional of the target field $A^{-}$, via Wilson lines. Its construction is well documented in the literature and will be briefly discussed in appendix B, where we show that

$$
G_{a b}^{--}\left(x^{+}, \boldsymbol{x} ; y^{+}, \boldsymbol{y} ; p^{+}\right)=\frac{1}{\left(p^{+}\right)^{2}} \partial_{\boldsymbol{x}}^{i} \partial_{\boldsymbol{y}}^{i} G_{a b}\left(x^{+}, \boldsymbol{x} ; y^{+}, \boldsymbol{y} ; p^{+}\right)+\frac{\mathrm{i}}{\left(p^{+}\right)^{2}} \delta_{a b} \delta\left(x^{+}-y^{+}\right) \delta_{\boldsymbol{x} \boldsymbol{y}} .
$$

Here, $G_{a b}$ is the 'scalar' propagator, defined as the solution to the following equation

$$
\left[2 \mathrm{i} p^{+}\left(\partial_{x}^{-}-\mathrm{i} g A^{-}(x)\right)+\nabla_{\perp x}^{2}\right]_{a c} G_{c b}\left(x^{+}, \boldsymbol{x} ; y^{+}, \boldsymbol{y} ; p^{+}\right)=\mathrm{i} \delta_{a b} \delta\left(x^{+}-y^{+}\right) \delta_{\boldsymbol{x} \boldsymbol{y}},
$$

with Feynman prescription for the pole at the mass-shell. This prescription ensures that modes with positive (negative) values of $p^{+}$propagate forward (backward) in time (see e.g. eq. (B.8)). For definiteness, we shall refer to the two pieces in the r.h.s. of eq. (2.8) as the 'radiative piece' and respectively the 'Coulomb piece' of the gluon propagator.

eq. (2.9) exhibits the eikonal coupling between the large component $p^{+}$of the $4-$ momentum of the gluon and the conjugate component $A^{-}$of the color field of the target, and also the transverse dynamics responsible for quantum diffusion. Given the formal analogy between this equation and the Schrödinger equation for a non-relativistic particle in two spatial dimensions, it is clear that its solution can be written as a path integral. Namely, for $p^{+}>0$ and hence $x^{+}>y^{+}$, one has ${ }^{5}$

$$
\begin{aligned}
G\left(x^{+}, \boldsymbol{x} ; y^{+}, \boldsymbol{y} ; p^{+}\right) & =\frac{1}{2 p^{+}} \mathcal{G}\left(x^{+}, \boldsymbol{x} ; y^{+}, \boldsymbol{y} ; p^{+}\right), \\
\mathcal{G}\left(x^{+}, \boldsymbol{x} ; y^{+}, \boldsymbol{y} ; p^{+}\right) & =\int[\mathcal{D} \boldsymbol{r}(t)] \exp \left\{\mathrm{i} \frac{p^{+}}{2} \int_{y^{+}}^{x^{+}} \mathrm{d} t \dot{\boldsymbol{r}}^{2}(t)\right\} U_{x^{+} y^{+}}^{\dagger}[\boldsymbol{r}(t)],
\end{aligned}
$$

where one integrates over paths $\boldsymbol{r}(t)$ with boundary conditions $\boldsymbol{r}\left(y^{+}\right)=\boldsymbol{y}$ and $\boldsymbol{r}\left(x^{+}\right)=\boldsymbol{x}$. For $p^{+}<0$ (and hence $x^{+}<y^{+}$), the propagator can be computed by using the following symmetry property, which follows from eq. (2.9) together with the Feynman prescription:

$$
G_{a b}\left(x^{+}, \boldsymbol{x} ; y^{+}, \boldsymbol{y} ; p^{+}\right)=G_{b a}\left(y^{+}, \boldsymbol{y} ; x^{+}, \boldsymbol{x} ;-p^{+}\right),
$$

By exploiting the above properties, one can limit the time integrals in eq. (2.4) to $-\infty<$ $t_{1}<t_{2}<\infty$, while simultaneously restricting the $p^{+}$integral to the positive side of the strip, $x \Lambda<p^{+}<\Lambda$, and multiplying the result by two. More precisely, we have here in mind the integral over the 'radiation' piece of the propagator (2.8), which is non-local in time. The local, Coulomb, piece must be treated separately.

\footnotetext{
${ }^{5}$ The 'reduced propagator' $\mathcal{G}$ is formally the same as the non-relativistic evolution operator.
} 
Note finally that there is no ambiguity concerning the ordering of the various factors within the integrand of eq. (2.4): (i) the two charge operators act at different times, $t_{1}$ and $t_{2}$, so they commute with each other; ( $\left.i i\right)$ the radiation piece of the propagator involves the background field $A^{-}(t)$ only at intermediate times $t$, between $t_{1}$ and $t_{2}$, so it commutes with any of the two functional derivatives; (iii) the Coulomb piece is local not only in time, but also in color.

The structure of the evolution Hamiltonian (2.4) looks both simple and intuitive: this operator does precisely what it is expected to do, namely, it generates the evolution of an $S$-matrix like (2.2) via the emission and the reabsorption of a soft gluon by any of the color sources within the projectile. But this apparent simplicity hides several subtleties which show up when trying to use this Hamiltonian in practice. These subtleties will be discussed in the next subsection, where we shall derive an alternative form for the evolution Hamiltonian - more precisely, for its action on a generic operator $\hat{\mathcal{O}}\left[A^{-}\right]$- which is more convenient in practice, especially for an extended target.

\subsection{Virtual corrections and probability conservation}

The purpose of this subsection is to render the Hamiltonian (2.4) 'less formal'. First, we shall argue that, in order to be well defined, this operator must be supplemented with an adiabatic prescription for switching off the interactions at large times. Second, we shall discuss a sum-rule for the free LC gauge propagator, which ensures probability conservation and also the cancellation of ultraviolet and infrared divergences between the 'radiative' piece and the 'Coulomb' piece of the Hamiltonian. Finally, we shall derive an alternative expression for the action of $\Delta H$ where this cancellation occurs locally in time and probability conservation becomes manifest.

Throughout this paper, we shall assume that the target is localized in $x^{+}$, within the longitudinal ${ }^{6}$ strip at $0<x^{+}<L$, so the collision has a finite duration $\Delta x^{+} \sim L$. The scattering amplitude can only be affected by gluon emissions which occur sufficiently close to this interaction region, within a time interval $\Delta x^{+} \sim \tau_{c o h}$. (We recall that the 'coherence time' $\tau_{c o h} \equiv 2 p^{+} / p_{\perp}^{2}$ is the typical lifetime of the fluctuation.) Vice-versa, virtual fluctuations in the wave function of the projectile which occur very far away from the interaction region, either in the remote past or the remote future, should have no influence on the evolution of the $S$-matrix. As we shall see, this property is correctly encoded in the present formalism, but it involves delicate cancellations between various terms, which might be invalidated by careless manipulations at intermediate stages. It turns out that a proper way to deal with this problem is to adiabatically switch off the interactions at very large times $\left|x^{+}\right| \gg \tau_{c o h}[13,57]$. (Other, less smooth, prescriptions, like a sharp cutoff on $\left|x^{+}\right|$, could induce spurious radiation and thus alter the Fock space of the projectile.) To that aim, we shall supplement each functional derivative within $\Delta H$

\footnotetext{
${ }^{6}$ An interval $\Delta x^{+}$is 'longitudinal' from the viewpoint of the target (a left mover), but 'temporal' from that of the projectile (a right mover). In what follows, we shall often mix the two viewpoints and the respective terminologies. The precise meaning should be clear from the context.
} 
with an exponential attenuation factor,

$$
J^{a}(t, \boldsymbol{r}) \rightarrow J^{a}(t, \boldsymbol{r}) \mathrm{e}^{-\epsilon|t|},
$$

where $\epsilon$ should be much smaller than $1 / \tau_{\text {coh }}$. The physical predictions will not be sensitive to the precise value of $\epsilon$ because the limit $\epsilon \rightarrow 0$ of the final results, as obtained after performing the integrals over the emission times $t_{1}$ and $t_{2}$, is indeed well defined.

With this adiabatic switch-off, the free LC gauge propagator $G_{0}^{--}$, eq. (B.7), obeys the following sum-rule, with paramount consequences for what follows:

$$
\int \mathrm{d} t_{1} \int \mathrm{d} t_{2} G_{0}^{--}\left(t_{2}-t_{1}, \boldsymbol{r} ; p^{+}\right) \mathrm{e}^{-\epsilon\left(\left|t_{1}\right|+\left|t_{2}\right|\right)}=0 .
$$

This will be demonstrated in appendix C, where we show that the l.h.s. of eq. (2.13) is a quantity of $\mathcal{O}(\epsilon)$ and hence vanishes when $\epsilon \rightarrow 0$. A simple way to understand this cancellation is to notice that the integral over $\Delta t \equiv t_{2}-t_{1}$ isolates the Fourier component with $p^{-}=0$, which vanishes because $G_{0}^{--}(p) \propto p^{-}$, cf. eq. (B.7). But this property holds only for the complete propagator, $G_{0}^{--}=G_{0, \text { rad }}^{--}+G_{0, \text { Coul }}^{--}$, as obtained after adding its radiative and Coulomb pieces. In the presence of a background field, we have to distinguish between these two pieces, since they are differently dressed by the background, cf. eq. (2.8). Taken separately, the radiative piece $G_{0, \text { rad }}^{--}$and the Coulomb piece $G_{0, \text { Coul }}^{--}$generate contributions $\propto 1 / \epsilon$ to the l.h.s. of eq. (2.13), which however cancel, together with the finite terms of $\mathcal{O}(1)$, in their sum (see appendix $\mathrm{C}$ ).

In view of the above, the sum-rule (2.13) is expected to be important for the limit $A^{-} \rightarrow$ 0 of our formalism. In that limit, it ensures an important property, that we now explain. As previously mentioned, quantum fluctuations which are not measured by the collision should not matter for the evolution of the $S$-matrix. Consider in particular the situation where, after acting with $\Delta H$ on some generic $S$-matrix $\hat{\mathcal{O}}$ (to produce the fluctuation), one sets $A^{-}=0$, so that there is no scattering. Without scattering, the evolution cannot be measured (the $S$-matrix must be equal to one both before and after the evolution), hence the action of $\Delta H$ must vanish:

$$
\left.\Delta H \hat{\mathcal{O}}\right|_{A^{-}=0}=0 .
$$

This is precisely ensured by the identity (2.13), as it can be easily seen: the action of the functional derivatives on $\hat{\mathcal{O}}$ becomes independent of time after we set $A^{-}=0$ (since all the Wilson lines are replaced by the unity matrix). Accordingly, the result of first acting with $\Delta H$ on any $\hat{\mathcal{O}}$ and then letting $A^{-} \rightarrow 0$ is indeed proportional to the integral in the l.h.s. of eq. (2.13).

These properties, eqs. (2.13) and (2.14), allows one to compute the action of $\Delta H$ on $\hat{\mathcal{O}}$ in an alternative way, where the Coulomb piece of the propagator is not explicitly present anymore and the cancellation of would-be divergent contributions occurs quasi-locally in time. Namely, eq. (2.14) implies, with obvious notations,

$$
\left.\Delta H_{\text {Coul }} \hat{\mathcal{O}}\right|_{A^{-}=0}=-\left.\Delta H_{\text {rad }} \hat{\mathcal{O}}\right|_{A^{-}=0} .
$$


Also, as we shall shortly demonstrate, the action of the Coulomb piece of the Hamiltonian on any observable $\hat{\mathcal{O}}$ amounts to

$$
\Delta H_{\text {Coul }} \hat{\mathcal{O}}=\left(\left.\Delta H_{\text {Coul }} \hat{\mathcal{O}}\right|_{A^{-}=0}\right) \hat{\mathcal{O}}=-\left(\left.\Delta H_{\text {rad }} \hat{\mathcal{O}}\right|_{A^{-}=0}\right) \hat{\mathcal{O}}
$$

where the second equality follows after using eq. (2.15). By using the above, one can write

$$
\Delta H \hat{\mathcal{O}}=\left[\Delta H_{\text {rad }}+\Delta H_{\text {Coul }}\right] \hat{\mathcal{O}}=\left[\Delta H_{\text {rad }}-\left(\left.\Delta H_{\text {rad }} \hat{\mathcal{O}}\right|_{A^{-}=0}\right)\right] \hat{\mathcal{O}}
$$

or, less formally,

$$
\begin{aligned}
\Delta H \hat{\mathcal{O}}\left[A^{-}\right]=\int_{x \Lambda}^{\Lambda} \frac{\mathrm{d} p^{+}}{2 \pi} \int_{-\infty}^{\infty} \mathrm{d} t_{2} \int_{-\infty}^{t_{2}} \mathrm{~d} t_{1} \mathrm{e}^{-\epsilon\left(\left|t_{1}\right|+\left|t_{2}\right|\right)} \times \\
\quad \times \int \mathrm{d}^{2} \boldsymbol{r}_{2} \int \mathrm{d}^{2} \boldsymbol{r}_{1}\left[\mathcal{H}-\left(\left.\mathcal{H} \hat{\mathcal{O}}\right|_{A^{-}=0}\right)\right] \hat{\mathcal{O}}
\end{aligned}
$$

where $\mathcal{H}$ is a Hamiltonian density built with the 'radiation' piece of the propagator alone:

$$
\mathcal{H}\left(t_{2}, \boldsymbol{r}_{2} ; t_{1}, \boldsymbol{r}_{1} ; p^{+}\right)\left[A^{-}\right] \equiv \frac{1}{\left(p^{+}\right)^{2}} J^{a}\left(t_{2}, \boldsymbol{r}_{2}\right)\left[\partial_{\boldsymbol{r}_{2}}^{i} \partial_{\boldsymbol{r}_{1}}^{i} G_{a b}\left(t_{2}, \boldsymbol{r}_{2} ; t_{1}, \boldsymbol{r}_{1} ; p^{+}\right)\right] J^{b}\left(t_{1}, \boldsymbol{r}_{1}\right)
$$

In eq. (2.19), the transverse derivatives act only on the 'scalar' propagator. In particular,

$$
\left.\mathcal{H} \hat{\mathcal{O}}\right|_{A^{-}=0}=\frac{1}{\left(p^{+}\right)^{2}}\left[\partial_{\boldsymbol{r}_{2}}^{i} \partial_{\boldsymbol{r}_{1}}^{i} G_{0}\left(t_{2}-t_{1}, \boldsymbol{r}_{2}-\boldsymbol{r}_{1} ; p^{+}\right)\right]\left(\left.J^{a}\left(t_{2}, \boldsymbol{r}_{2}\right) J^{a}\left(t_{1}, \boldsymbol{r}_{1}\right) \hat{\mathcal{O}}\right|_{A^{-}=0}\right)
$$

with $G_{0}$ the free propagator (B.8). Notice that the r.h.s. of eq. (2.18) cannot be written as the action of a linear operator on $\hat{\mathcal{O}}$. Hence, this equation does not provide an alternative expression for the Hamiltonian $\Delta H$, but rather a new method for computing its action on a generic observable.

Using $\left.\hat{\mathcal{O}}\right|_{A^{-}=0}=1$, one sees that the property (2.14) is now satisfied locally in time, that is, it is already verified by the integrand in eq. (2.18). This allows for a natural probabilistic interpretation: the term $\mathcal{H} \hat{\mathcal{O}}$ describes the change in the $S$-matrix associated with a real emission which occurrs during the time interval from $t_{1}$ to $t_{2}$; the virtual term $-\left(\left.\mathcal{H} \hat{\mathcal{O}}\right|_{A^{-}=0}\right) \hat{\mathcal{O}}$ represents the reduction in the probability that the projectile remain in its original state during that time interval. The local (in time) version of (2.14) is then the expression of probability conservation.

To better appreciate the advantages of eq. (2.18) over the direct use of eq. (2.4), let us consider the action of $\Delta H_{\text {Coul }}$ in more detail. (This will also allow us to verify the first equality in eq. (2.16).) What we would like to show is that any operator $\hat{\mathcal{O}}$ is an eigenstate of $\Delta H_{\text {Coul }}$, but with an ill-define eigenvalue, which suffers from both infrared (large time and small $p^{+}$) and ultraviolet (small $\left|\boldsymbol{r}_{2}-\boldsymbol{r}_{1}\right|$, or high $p_{\perp}$ ) divergences. Chosing $\hat{\mathcal{O}}=\hat{S}_{\boldsymbol{x} \boldsymbol{y}}$ 
for definiteness (this brings no loss in generality), we can write (cf. eq. (2.8))

$$
\begin{aligned}
\Delta H_{\text {Coul }} \hat{S}_{\boldsymbol{x} \boldsymbol{y}} & =\int_{x \Lambda}^{\Lambda} \frac{\mathrm{d} p^{+}}{2 \pi} \int_{t_{1}, t_{2}} \int_{\boldsymbol{r}_{1}, \boldsymbol{r}_{2}} \mathrm{e}^{-\epsilon\left(\left|t_{1}\right|+\left|t_{2}\right|\right)} \frac{\mathrm{i}}{\left(p^{+}\right)^{2}} \delta_{t_{2} t_{1}} \delta_{\boldsymbol{r}_{1} \boldsymbol{r}_{2}} J^{a}\left(t_{2}, \boldsymbol{r}_{2}\right) J^{a}\left(t_{1}, \boldsymbol{r}_{1}\right) \hat{S}_{\boldsymbol{x} \boldsymbol{y}} \\
& =-\frac{\mathrm{i} g^{2} C_{F}}{2 \pi} \int_{x \Lambda}^{\Lambda} \frac{\mathrm{d} p^{+}}{\left(p^{+}\right)^{2}} \int \mathrm{d} t \mathrm{e}^{-2 \epsilon|t|} \int \mathrm{d}^{2} \boldsymbol{r}\left(\delta_{\boldsymbol{r} \boldsymbol{x}}+\delta_{\boldsymbol{r} \boldsymbol{y}}\right) \delta_{\boldsymbol{r} \boldsymbol{r}} \hat{S}_{\boldsymbol{x} \boldsymbol{y}} \\
& =-\frac{\mathrm{i} g^{2} C_{F}}{\pi}\left[\delta_{\boldsymbol{r} \boldsymbol{r}} \frac{1}{\epsilon} \int_{x \Lambda}^{\Lambda} \frac{\mathrm{d} p^{+}}{\left(p^{+}\right)^{2}}\right] \hat{S}_{\boldsymbol{x} \boldsymbol{y}} .
\end{aligned}
$$

Because of the ultra-local nature of the Coulomb propagator $\propto \delta_{t_{2} t_{1}} \delta_{\boldsymbol{r}_{1} \boldsymbol{r}_{2}}$, the two functional derivatives must act on a same Wilson line within $\hat{S}_{\boldsymbol{x} \boldsymbol{y}}$, either the quark one at $\boldsymbol{x}$ or the antiquark one at $\boldsymbol{y}$. This feature, together with identities like

$$
J^{a}\left(t, \boldsymbol{r}_{2}\right) J^{a}\left(t, \boldsymbol{r}_{1}\right) V_{\boldsymbol{x}}^{\dagger}=-g^{2} C_{F} \delta_{\boldsymbol{r}_{1} \boldsymbol{x}} \delta_{\boldsymbol{r}_{2} \boldsymbol{x}} V_{\boldsymbol{x}}^{\dagger},
$$

explains why the result is again proportional to $\hat{S}_{\boldsymbol{x} \boldsymbol{y}}$. But for the very same reason, the proportionality coefficient exhibits several types of divergences, as anticipated: a largetime divergence as $\epsilon \rightarrow$, a small- $p^{+}$divergence when $x \rightarrow 0$, and a transverse 'tadpole' $\delta_{\boldsymbol{r} \boldsymbol{r}}=\int\left[\mathrm{d}^{2} \boldsymbol{p} /\left(2 \pi^{2}\right)\right]$. Being independent of $A^{-}$, this coefficient is necessarily the same as the limit $A^{-} \rightarrow 0$ of $\Delta H_{\text {Coul }} \hat{S}_{x y}$, in agreement with eq. (2.16). Clearly, a similar argument holds for any observable $\hat{\mathcal{O}}$.

The above discussion shows that the action of the Coulomb piece of $\Delta H$ generates severe divergences. By virtue of eq. (2.13), there divergences are guaranteed to cancel against similar ones generated by the radiation piece, but only after performing the two time integrations. This cancellation can be explicitly verified whenever one is able to perform the time integrations, as in the case of a shockwave target to be discussed in section 3. But even in such a case, the calculation of the finite terms is quite subtle and relies in an essential way on the use of the adiabatic prescription (see e.g. section 3.1). By contrast, the calculations based on eq. (2.18) are more robust, because the potential divergences cancel between the 'real' and 'virtual' terms quasi-locally in time, so one is not sensitive to the regularization prescription used for the time integrations. This second method becomes particularly useful in those cases where one is not able to explicitly perform the time integrals, like that of an extended target to be discussed in section 4 .

\section{A shockwave target: recovering the JIMWLK Hamiltonian}

In this section, we shall specialize the general formalism developed so far to the case where the target is a 'shockwave'. By this, we more precisely mean a target which looks localized in $x^{+}$on the resolution scale set by the lifetime of the quantum fluctuations. For this case, we will be able to explicitly perform the time integrations which appear in eq. (2.4) and thus recover the JIMWLK Hamiltonian [21-29], as expected. Besides giving us more confidence with the use of eq. (2.4) in practice, the subsequent manipulations will also 
illustrate some of the subtleties discussed in section 2.2, notably the role of the adiabatic prescription and the cancellation of the ill-defined contributions between the 'radiation' piece and the 'Coulomb' piece of $\Delta H$.

More precisely, the physical problem that we here have in mind is 'dense-dilute' (e.g. proton-nucleus) scattering in the high-energy regime where the longitudinal extent $\Delta x^{+} \equiv$ $L$ of the dense target is much smaller than the coherence time $\tau_{c o h}=2 p^{+} / p_{\perp}^{2}$ of the typical gluons fluctuations associated with the evolution of the projectile: $\tau_{c o h} \gg L$. This condition involves both the 'energy' (actually, LC longitudinal momentum) $p^{+}$and the transverse momentum $p_{\perp}$ of the gluon fluctuations. In practice, $p_{\perp}$ is at least as large as the target saturation momentum $Q_{s}$, since this is the typical transverse momentum acquired by either the soft gluon, or its parent parton, via interactions with the target (see e.g. [12, 35, 36]). Hence, the 'shockwave condition' can be written as a lower limit on the gluon energy: $p^{+} \gg \omega_{c}$, with

$$
\omega_{c} \equiv Q_{s}^{2} L
$$

This limiting energy $\omega_{c}$ is an intrinsic scale of the target and grows with the target size like $\omega_{c} \sim L^{2}$ (since $Q_{s}^{2} \propto L$ ). To have a significant phase-space for the high-energy evolution, the energy $p_{0}^{+} \equiv E$ of the incoming projectile must be considerably larger than $\omega_{c}$, such that $\bar{\alpha} \ln \left(E / \omega_{c}\right) \gtrsim 1$ with $\bar{\alpha} \equiv \alpha_{s} N_{c} / \pi$ assumed to be small $(\bar{\alpha} \ll 1)$.

\subsection{Performing the time integrations}

What is special about the shockwave (SW) target, is that the probability for a gluon to be emitted or absorbed inside the target is negligible, ${ }^{7}$ since suppressed by a factor $L / \tau_{c o h} \ll 1$. This physical statement is boost invariant, but the mathematics becomes simpler by working in the 'target infinite momentum frame', i.e. a frame in which the nucleus is ultrarelativistic and it looks like a 'pancake' (our intuitive representation of a $\mathrm{SW})$. In such a frame, the target can be effectively treated as a $\delta$-function at $x^{+}=0$. This drastically simplifies the structure of the background field propagator and the action of the functional derivatives on the Wilson lines.

Namely, assuming the SW to be localized near $x^{+}=0$, one can easily show that the path integral in eq. (2.10) reduces to (for $p^{+}>0$ and hence $x^{+}>y^{+}$; see appendix $\mathrm{B}$ for details)

$$
\begin{aligned}
G\left(x^{+}, \boldsymbol{x} ; y^{+}, \boldsymbol{y} ; p^{+}>0\right)=G_{0}\left(x^{+}-y^{+}, \boldsymbol{x}-\boldsymbol{y} ; p^{+}\right)\left[\theta\left(x^{+}\right) \theta\left(y^{+}\right)+\theta\left(-x^{+}\right) \theta\left(-y^{+}\right)\right] \\
+2 p^{+} \theta\left(x^{+}\right) \theta\left(-y^{+}\right) \int_{\boldsymbol{z}} G_{0}\left(x^{+}, \boldsymbol{x}-\boldsymbol{z} ; p^{+}\right) U_{\boldsymbol{z}}^{\dagger} G_{0}\left(-y^{+}, \boldsymbol{z}-\boldsymbol{y} ; p^{+}\right)
\end{aligned}
$$

where $G_{0}$ is the free propagator (B.8), $U_{\boldsymbol{z}}^{\dagger}$ is the adjoint Wilson line introduced in eq. (2.1), and $\int_{\boldsymbol{z}} \equiv \int \mathrm{d}^{2} \boldsymbol{z}$. The physical interpretation of eq. (3.2) is quite transparent: when $x^{+}$and $y^{+}$are both positive, or both negative, the gluon does not cross the $\mathrm{SW}$, so it propagates freely; when $x^{+}$and $y^{+}$are on opposite sides of the SW, the gluon propagates freely from

\footnotetext{
${ }^{7}$ Strictly speaking, this statement is gauge-dependent, but it is indeed correct in the gauge $a^{+}=0$ that we currently use; see e.g. the discussion in [58].
} 
the initial point up to the SW, then it crosses the latter at some transverse position $\boldsymbol{z}$, thus accumulating a color precession represented by the Wilson line $U_{\boldsymbol{z}}^{\dagger}$, then it moves freely again, up to the final point.

Furthermore, since gluons cannot be emitted or absorbed inside the SW, the action of the functional derivative $J_{\boldsymbol{x}}^{a}(t)$ on the Wilson lines is piecewise independent of time. Indeed for any negative value of the time argument, one has (compare to eq. (2.6))

$$
J_{\boldsymbol{x}}^{a}(t<0) U_{\boldsymbol{z}}^{\dagger}=\mathrm{i} g \delta_{\boldsymbol{z} \boldsymbol{x}} U_{\boldsymbol{z}}^{\dagger}(\infty, t) T^{a} U_{\boldsymbol{z}}^{\dagger}(t,-\infty)=\mathrm{i} g \delta_{\boldsymbol{z} \boldsymbol{x}} U_{\boldsymbol{z}}^{\dagger} T^{a} \equiv R_{\boldsymbol{x}}^{a} U_{\boldsymbol{z}}^{\dagger},
$$

where we have used $U_{\boldsymbol{z}}^{\dagger}(t,-\infty)=1$ and $U_{\boldsymbol{z}}^{\dagger}(\infty, t)=U_{\boldsymbol{z}}^{\dagger}(\infty,-\infty) \equiv U_{\boldsymbol{z}}^{\dagger}$ for $t<0$ and a target field localized at $x^{+}=0$. Similarly, for a positive value $t>0$, one can write

$$
J_{\boldsymbol{x}}^{a}(t>0) U_{\boldsymbol{z}}^{\dagger}=\mathrm{i} g \delta_{\boldsymbol{z} \boldsymbol{x}} U_{\boldsymbol{z}}^{\dagger}(\infty, t) T^{a} U_{\boldsymbol{z}}^{\dagger}(t,-\infty)=\mathrm{i} g \delta_{\boldsymbol{z} \boldsymbol{x}} T^{a} U_{\boldsymbol{z}}^{\dagger} \equiv L_{\boldsymbol{x}}^{a} U_{\boldsymbol{z}}^{\dagger},
$$

The above equations have introduced the 'right' and 'left' functional derivatives, $R_{x}^{a}$ and respectively $L_{\boldsymbol{x}}^{a}$, which act on the Wilson lines as infinitesimal color rotations of the right, respectively on the left, and measure the color charge density in the projectile prior, respectively after, the collision. They are related by the condition $L_{\boldsymbol{x}}^{a}=U_{\boldsymbol{x}}^{\dagger a b} R_{\boldsymbol{x}}^{b}$, which expresses the color rotation acquired by a color current which crosses the shockwave.

The fact that the r.h.s.'s of eqs. (3.3)-(3.4) are independent of time allows us to perform the time integrations directly at the level of the evolution Hamiltonian (2.4), that is, before acting with $\Delta H$ on the observable. To that aim, we need to distinguish three regions for the time integrations:

(i) $-\infty<t_{1}<0$ and $0<t_{2}<\infty$ : the evolution gluon crosses the SW. After using eqs. (3.3)-(3.4) for the action of the functional derivatives, one sees that the respective contribution to $\Delta H$, denoted as $\Delta H_{R L}$, simplifies to

$$
\Delta H_{R L}=\int_{\boldsymbol{x}, \boldsymbol{y}} L_{\boldsymbol{x}}^{a} R_{\boldsymbol{y}}^{b} \int_{x \Lambda}^{\Lambda} \frac{\mathrm{d} p^{+}}{2 \pi} \frac{1}{\left(p^{+}\right)^{2}} \int_{-\infty}^{0} \mathrm{~d} t_{1} \int_{0}^{\infty} \mathrm{d} t_{2} \partial_{\boldsymbol{x}}^{i} \partial_{\boldsymbol{y}}^{i} G_{a b}\left(t_{2}, \boldsymbol{x} ; t_{1}, \boldsymbol{y} ; p^{+}\right),
$$

where the adiabatic prescriptions are implicit (they will be exhibited when needed) and, cf. eq. (3.2),

$$
\partial_{\boldsymbol{x}}^{i} \partial_{\boldsymbol{y}}^{i} G_{a b}\left(t_{2}, \boldsymbol{x} ; t_{1}, \boldsymbol{y} ; p^{+}\right)=2 p^{+} \int_{\boldsymbol{z}} \partial_{\boldsymbol{x}}^{i} G_{0}\left(t_{2}, \boldsymbol{x}-\boldsymbol{z} ; p^{+}\right)\left(U_{\boldsymbol{z}}^{\dagger}\right)_{a b} \partial_{\boldsymbol{y}}^{i} G_{0}\left(-t_{1}, \boldsymbol{z}-\boldsymbol{y} ; p^{+}\right)
$$

Due to the factorized structure of the background field propagator (3.6), the two time integrations are independent of each other. To be specific, consider the integral over $t_{2}$. This involves

$$
\begin{aligned}
\int_{0}^{\infty} \mathrm{d} t_{2} \partial_{\boldsymbol{x}}^{i} G_{0}\left(t_{2}, \boldsymbol{x}-\boldsymbol{z} ; p^{+}\right) & =\frac{-\mathrm{i}}{2 p^{+}} \int \frac{\mathrm{d}^{2} \boldsymbol{p}}{(2 \pi)^{2}} p^{i} \mathrm{e}^{\mathrm{i} \boldsymbol{p} \cdot(\boldsymbol{x}-\boldsymbol{z})} \int_{0}^{\infty} \mathrm{d} t_{2} \mathrm{e}^{-\mathrm{i} \frac{p_{\perp}^{2}}{2 p^{+}} t_{2}} \mathrm{e}^{-\epsilon t_{2}} \\
& =-\int \frac{\mathrm{d}^{2} \boldsymbol{p}}{(2 \pi)^{2}} \frac{p^{i}}{p_{\perp}^{2}} \mathrm{e}^{\mathrm{i} \boldsymbol{p} \cdot(\boldsymbol{x}-\boldsymbol{z})}=\frac{\mathrm{i}}{2 \pi} \frac{x^{i}-z^{i}}{(\boldsymbol{x}-\boldsymbol{z})^{2}}
\end{aligned}
$$


The final result is recognized as the Weizsäcker-Williams field created at $\boldsymbol{z}$ by a pointlike source at $\boldsymbol{x}$. Note that the complex exponential in the integral over $t_{2}$ has restricted the respective phase-space to an interval of order $\tau_{c o h}=2 p^{+} / p_{\perp}^{2}$ after the SW. A similar conclusion holds for the emission time $t_{1}$, which is restricted to an interval $\sim \tau_{\text {coh }}$ before the SW. The respective integral yields

$$
\int_{-\infty}^{0} \mathrm{~d} t_{1} \partial_{\boldsymbol{y}}^{i} G_{0}\left(-t_{1}, \boldsymbol{z}-\boldsymbol{y} ; p^{+}\right)=\frac{\mathrm{i}}{2 \pi} \frac{y^{i}-z^{i}}{(\boldsymbol{y}-\boldsymbol{z})^{2}} .
$$

Importantly, the final results in eqs. (3.7)-(3.8) are independent of $p^{+}$. In both cases, this is due to a cancellation between the factor $1 / p^{+}$implicit in the free propagator $G_{0}$ and the phase-space factor $2 p^{+} / p_{\perp}^{2}$ produced by the time integral. As a consequence, the ensuing integral over $p^{+}$in eq. (3.5) is logarithmic: $\int\left(\mathrm{d} p^{+} / p^{+}\right)=\ln (1 / x)$. Putting all together, one finds

$$
\Delta H_{R L}=-\ln \frac{1}{x} \frac{1}{(2 \pi)^{3}} \int_{\boldsymbol{x} \boldsymbol{y} \boldsymbol{z}} \mathcal{K}_{\boldsymbol{x} \boldsymbol{y} \boldsymbol{z}}\left(2 L_{\boldsymbol{x}}^{a} U_{\boldsymbol{z}}^{\dagger a b} R_{\boldsymbol{y}}^{b}\right),
$$

with the following notations:

$$
\mathcal{K}_{\boldsymbol{x} \boldsymbol{y} \boldsymbol{z}} \equiv \mathcal{K}_{\boldsymbol{x} \boldsymbol{z}}^{i} \mathcal{K}_{\boldsymbol{y} \boldsymbol{z}}^{i}, \quad \mathcal{K}_{\boldsymbol{x} \boldsymbol{z}}^{i} \equiv \frac{(\boldsymbol{x}-\boldsymbol{z})^{i}}{(\boldsymbol{x}-\boldsymbol{z})^{2}}
$$

(ii) $-\infty<t_{1} \leq t_{2}<0$ : the evolution gluon is emitted and reabsorbed prior to the SW. In this case, both functional derivatives within $\Delta H$ act as 'right' derivatives, cf. eq. (3.3). Also, the gluon propagator reduces to the free propagator $G_{0}^{--}$, as shown in eq. (B.7). Consider first the 'radiation' piece of this propagator, which gives

$$
\Delta H_{R R}^{\mathrm{rad}}=\int_{\boldsymbol{x}, \boldsymbol{y}} R_{\boldsymbol{x}}^{a} R_{\boldsymbol{y}}^{a} \int_{x \Lambda}^{\Lambda} \frac{\mathrm{d} p^{+}}{2 \pi} \frac{1}{\left(p^{+}\right)^{2}} \int_{-\infty}^{0} \mathrm{~d} t_{2} \int_{-\infty}^{t_{2}} \mathrm{~d} t_{1} \partial_{\boldsymbol{x}}^{i} \partial_{\boldsymbol{y}}^{i} G_{0}\left(t_{2}-t_{1}, \boldsymbol{x}-\boldsymbol{y} ; p^{+}\right) .
$$

The time integrations involve (with the shorthand notation $p^{-} \equiv p_{\perp}^{2} / 2 p^{+}$)

$$
\begin{aligned}
\int_{-\infty}^{0} \mathrm{~d} t_{2} \int_{-\infty}^{t_{2}} \mathrm{~d} t_{1} \mathrm{e}^{-\mathrm{i} p^{-}\left(t_{2}-t_{1}\right)} \mathrm{e}^{\epsilon\left(t_{1}+t_{2}\right)} & =\int_{-\infty}^{0} \mathrm{~d} t_{2} \mathrm{e}^{-\mathrm{i} p^{-} t_{2}+\epsilon t_{2}} \frac{\mathrm{e}^{\mathrm{i} p^{-} t_{2}+\epsilon t_{2}}}{\mathrm{i} p^{-}+\epsilon} \\
& =\frac{1}{2 \epsilon} \frac{1}{\mathrm{i} p^{-}+\epsilon}=\frac{1}{2 \epsilon} \frac{1}{\mathrm{i} p^{-}}+\frac{1}{2\left(p^{-}\right)^{2}}+\mathcal{O}(\epsilon) .
\end{aligned}
$$

The use of the adiabatic prescription has been essential in obtaining the above result, as we now explain. The time separation $t_{2}-t_{1}$ is restricted by the oscillatory phase $\mathrm{e}^{-\mathrm{i} p^{-}\left(t_{2}-t_{1}\right)}$ to values of order $\tau_{c o h}=2 p^{+} / p_{\perp}^{2}$, but the central value $\left(t_{2}+t_{1}\right) / 2$ is only restricted by the adiabatic switch-off, so the corresponding integral yields an 'infrared' divergence proportional to $1 / \epsilon$. By itself, this divergence is pretty harmless, since ultimately cancelled by a similar contribution due to the Coulomb piece, as we shall see. What is more subtle though, is the obtention of the finite term accompanying the divergence (namely, the term $\propto 1 /\left(p^{-}\right)^{2}$ in eq. (3.12)): this term is correctly computed when using the adiabatic prescription, as above, but it would be mistreated by other regularizations, like a sharp 
cutoff on $\left|t_{2}+t_{1}\right|[13,57]$. Importantly, this finite contribution, which is the actual physical result, has been generated by values $t_{1}$ and $t_{2}$ which both lie in the vicinity of the interaction time $x^{+}=0$, within a distance of order $\tau_{c o h}$.

By using eq. (3.12) together with simple manipulations, one finds

$$
\Delta H_{R R}^{\mathrm{rad}}=\int_{\boldsymbol{x}, \boldsymbol{y}} R_{\boldsymbol{x}}^{a} R_{\boldsymbol{y}}^{a} \int_{x \Lambda}^{\Lambda} \frac{\mathrm{d} p^{+}}{2 \pi} \frac{1}{\left(p^{+}\right)^{2}}\left\{-\frac{\mathrm{i}}{2 \epsilon} \delta_{\boldsymbol{x} \boldsymbol{y}}+p^{+} \int \frac{\mathrm{d}^{2} \boldsymbol{p}}{(2 \pi)^{2}} \frac{\mathrm{e}^{\mathrm{i} \boldsymbol{p} \cdot(\boldsymbol{x}-\boldsymbol{y})}}{p_{\perp}^{2}}\right\} .
$$

The first term within the braces exhibits all types of divergences previously identified in relation with the Coulomb piece, cf. eq. (2.21). As demonstrated by the above calculation (and anticipated in section 2.2), such divergences are also generated by the 'radiation' piece after performing the time integrations. We shall shortly check that this singular term is cancelled by the respective 'Coulomb' contribution, in agreement with the discussion in section 2.2. Keeping only the second term in eq. (3.13), one finds that the respective integral over $p^{+}$is again logarithmic and yields

$$
\Delta H_{R R}=\ln \frac{1}{x} \frac{1}{(2 \pi)^{3}} \int_{\boldsymbol{x} \boldsymbol{y} \boldsymbol{z}} \mathcal{K}_{\boldsymbol{x} \boldsymbol{y} \boldsymbol{z}} R_{\boldsymbol{x}}^{a} R_{\boldsymbol{y}}^{a}
$$

where we have also used the identity

$$
\int \frac{\mathrm{d}^{2} \boldsymbol{p}}{(2 \pi)^{2}} \frac{\mathrm{e}^{\mathrm{i} \boldsymbol{p} \cdot(\boldsymbol{x}-\boldsymbol{y})}}{p_{\perp}^{2}}=\frac{1}{(2 \pi)^{2}} \int_{\boldsymbol{z}} \mathcal{K}_{\boldsymbol{x} \boldsymbol{y} \boldsymbol{z}}
$$

The above integral over $p_{\perp}$ develops a logarithmic infrared $\left(p_{\perp} \rightarrow 0\right)$ divergence, which is however harmless, as it disappears in the evolution of gauge-invariant quantities (see e.g. section 3.2 below).

For completeness, let us also consider the respective Coulomb contribution:

$$
\begin{aligned}
\Delta H_{R R}^{\text {Coul }} & =\mathrm{i} \int_{\boldsymbol{x}, \boldsymbol{y}} R_{\boldsymbol{x}}^{a} R_{\boldsymbol{y}}^{a} \int_{x \Lambda}^{\Lambda} \frac{\mathrm{d} p^{+}}{2 \pi} \frac{1}{\left(p^{+}\right)^{2}} \int_{-\infty}^{0} \mathrm{~d} t_{2} \int_{-\infty}^{0} \mathrm{~d} t_{1} \delta\left(t_{2}-t_{1}\right) \mathrm{e}^{\epsilon\left(t_{1}+t_{2}\right)} \delta_{\boldsymbol{x} \boldsymbol{y}} \\
& =\frac{\mathrm{i}}{2 \epsilon} \int_{\boldsymbol{x}} R_{\boldsymbol{x}}^{a} R_{\boldsymbol{x}}^{a} \int_{x \Lambda}^{\Lambda} \frac{\mathrm{d} p^{+}}{2 \pi} \frac{1}{\left(p^{+}\right)^{2}} .
\end{aligned}
$$

This precisely cancels the divergent piece in eq. (3.13), as anticipated. This cancellation illustrates a general argument developed in section 2.2, namely the fact that emissions which occur at large distances $\gg \tau_{c o h}$ from the interaction region cannot affect the scattering amplitude.

(iii) $0<t_{1} \leq t_{2}<\infty$ : the evolution gluon is emitted and reabsorbed after the SW. The calculation is entirely similar to that in the previous case, so we can write the final result without further discussion:

$$
\Delta H_{L L}=\ln \frac{1}{x} \frac{1}{(2 \pi)^{3}} \int_{\boldsymbol{x} \boldsymbol{y z}} \mathcal{K}_{\boldsymbol{x} \boldsymbol{y} \boldsymbol{z}} L_{\boldsymbol{x}}^{a} L_{\boldsymbol{y}}^{a}
$$


By combining the previous results (3.9), (3.14), and (3.17), one finds $\Delta H=$ $\ln (1 / x) H_{\text {JIMWLK }}$, with the JIMWLK Hamiltonian [21-29] (see also [59-62] for more recent derivations).

$$
H_{\mathrm{JIMWLK}}=\frac{1}{(2 \pi)^{3}} \int_{\boldsymbol{x} \boldsymbol{y} \boldsymbol{z}} \mathcal{K}_{\boldsymbol{x} \boldsymbol{y} \boldsymbol{z}}\left[R_{\boldsymbol{x}}^{a} R_{\boldsymbol{y}}^{a}+L_{\boldsymbol{x}}^{a} L_{\boldsymbol{y}}^{a}-2 L_{\boldsymbol{x}}^{a} U_{\boldsymbol{z}}^{\dagger a b} R_{\boldsymbol{y}}^{b}\right] .
$$

By using the unitarity of the Wilson lines together with the condition $L_{\boldsymbol{x}}^{a}=U_{\boldsymbol{x}}^{\dagger a b} R_{\boldsymbol{x}}^{b}$, one can rewrite the color structure in eq. (3.18) in the following form

$$
\begin{aligned}
R_{\boldsymbol{x}}^{a} R_{\boldsymbol{y}}^{a}+L_{\boldsymbol{x}}^{a} L_{\boldsymbol{y}}^{a}-2 L_{\boldsymbol{x}}^{a} U_{\boldsymbol{z}}^{\dagger a b} R_{\boldsymbol{y}}^{b} & =\left[L_{\boldsymbol{x}}^{a}-U_{\boldsymbol{z}}^{\dagger a b} R_{\boldsymbol{x}}^{b}\right]\left[L_{\boldsymbol{y}}^{a}-U_{\boldsymbol{z}}^{\dagger a c} R_{\boldsymbol{y}}^{c}\right] \\
& =\left[U_{\boldsymbol{x}}^{\dagger a b}-U_{\boldsymbol{z}}^{\dagger a b}\right] R_{\boldsymbol{x}}^{b}\left[U_{\boldsymbol{y}}^{\dagger a c}-U_{\boldsymbol{z}}^{\dagger a c}\right] R_{\boldsymbol{y}}^{c} .
\end{aligned}
$$

This makes it obvious that $H_{\text {JIMWLK }}$ vanishes when $A^{-}=0$, in agreement with eq. (2.14).

\subsection{The Balitsky-Kovchegov equation}

The simplest among the evolution equations generated by the JIMWLK Hamiltonian is the Balitsky-Kovchegov (BK) equation [19, 20], that is, the equation obeyed by the average $S$-matrix for a $q \bar{q}$ dipole. In what follow we shall present two different derivations for this equation: the standard one in the literature, where one directly acts with $H_{\text {JIMWLK }}$ on the dipole operator $\hat{S}_{\boldsymbol{x} \boldsymbol{y}}$, and the alternative one based on eq. (2.18), which distinguishes between 'real' and 'virtual' corrections. Clearly, the final result will be the same, but the comparison between these two methods will shed more light on the reorganization of the perturbation theory performed by the sum-rule (2.13) and also on the origin of the virtual terms in the B-JIMWLK equations.

(i) The standard approach. Consider the dipole-nucleus scattering in a Lorentz frame where the nuclear target carries most of the rapidity separation $Y$, so that the projectile is a bare dipole - a quark-antiquark pair without additional gluons. In this frame, the average $S$-matrix is computed as $[35,36]$

$$
\left\langle\hat{S}_{x \boldsymbol{y}}\right\rangle_{Y}=\int\left[D A^{-}\right] W_{Y}\left[A^{-}\right] \frac{1}{N_{c}} \operatorname{tr}\left(V_{\boldsymbol{x}}^{\dagger} V_{\boldsymbol{y}}\right),
$$

where the 'CGC weight function' $W_{Y}\left[A^{-}\right]$is a functional probability density describing the distribution of the color fields in the target (including its evolution up to rapidity $Y$ ). Let us now increase the rapidity separation, $Y \rightarrow Y+\Delta Y$, by giving an additional boost $\Delta Y$ to the projectile. Then the dipole evolves by emitting a soft gluon (from either the quark, or the antiquark), with longitudinal momentum fraction $x_{1}$ within the range $x<x_{1}<1$, where $\Delta Y=\ln 1 / x$. The ensuing evolution of the $S$-matrix is obtained by acting with the JIMWLK Hamiltonian on the bare scattering operator:

$$
\Delta\left\langle\hat{S}_{\boldsymbol{x} \boldsymbol{y}}\right\rangle_{Y}=\Delta Y\left\langle H_{\mathrm{JIMWLK}} \hat{S}_{\boldsymbol{x} \boldsymbol{y}}\right\rangle \equiv \int\left[D A^{-}\right] W_{Y}\left[A^{-}\right] H_{\mathrm{JIMWLK}} \hat{S}_{\boldsymbol{x} \boldsymbol{y}} .
$$

Using eq. (3.18) together with the differentiation rules in eqs. (3.3)-(3.4), one can easily deduce

$$
\left(H_{R R}+H_{L L}\right) \hat{S}_{\boldsymbol{x} \boldsymbol{y}}=-\frac{\bar{\alpha}}{2 \pi}\left(1-\frac{1}{N_{c}^{2}}\right) \int_{\boldsymbol{z}} \mathcal{M}_{\boldsymbol{x} \boldsymbol{y} \boldsymbol{z}} \hat{S}_{\boldsymbol{x} \boldsymbol{y}}
$$


for the contribution of the 'non-crossing' terms and, respectively,

$$
\begin{aligned}
H_{R L} \hat{S}_{\boldsymbol{x} \boldsymbol{y}} & =\frac{\alpha_{s}}{\pi^{2}} \int_{\boldsymbol{z}} \mathcal{M}_{\boldsymbol{x} \boldsymbol{y} \boldsymbol{z}} U_{\boldsymbol{z}}^{\dagger a b} \frac{1}{N_{c}} \operatorname{tr}\left(V_{\boldsymbol{x}}^{\dagger} t^{b} V_{\boldsymbol{y}} t^{a}\right) \\
& =\frac{\bar{\alpha}}{2 \pi} \int_{\boldsymbol{z}} \mathcal{M}_{\boldsymbol{x} \boldsymbol{y} \boldsymbol{z}}\left(\hat{S}_{\boldsymbol{x} \boldsymbol{z}} \hat{S}_{\boldsymbol{z} \boldsymbol{y}}-\frac{1}{N_{c}^{2}} \hat{S}_{\boldsymbol{x} \boldsymbol{y}}\right)
\end{aligned}
$$

for that of the 'crossing' one. In these equations, $\mathcal{M}_{\boldsymbol{x} \boldsymbol{y} \boldsymbol{z}}$ is the 'dipole kernel',

$$
\mathcal{M}_{x \boldsymbol{y} z} \equiv \mathcal{K}_{\boldsymbol{x} \boldsymbol{x} \boldsymbol{z}}+\mathcal{K}_{\boldsymbol{y} \boldsymbol{y} \boldsymbol{z}}-2 \mathcal{K}_{\boldsymbol{x} \boldsymbol{y} \boldsymbol{z}}=\frac{(\boldsymbol{x}-\boldsymbol{y})^{2}}{(\boldsymbol{x}-\boldsymbol{z})^{2}(\boldsymbol{z}-\boldsymbol{y})^{2}} .
$$

In the linear combination above, the positive terms $\mathcal{K}_{\boldsymbol{x} \boldsymbol{x} \boldsymbol{z}}$ and $\mathcal{K}_{\boldsymbol{y} \boldsymbol{y} \boldsymbol{z}}$ correspond to selfenergy corrections, i.e. graphs where both emissions are attached to a same fermion (the quark at $\boldsymbol{x}$ or the antiquark at $\boldsymbol{y}$ ), whereas the negative term $-2 \mathcal{K}_{\boldsymbol{x} \boldsymbol{y} \boldsymbol{z}}$ summarizes the two exchange graphs, where the gluon is emitted by the quark and absorbed by the antiquark, or vice-versa (see also figure 1 for similar graphs). Note that the leading behavior at large $z_{\perp}$, which is proportional to $1 / z_{\perp}^{2}$ for each of these individual graphs, has cancelled in their linear combination, with the result that $\mathcal{M}_{\boldsymbol{x} \boldsymbol{y z}} \sim 1 / z_{\perp}^{4}$ when $z_{\perp} \rightarrow \infty$. This decay is sufficiently fast to guarantee that the integral over $z$ is convergent in this limit. Similar cancellations occur for any projectile which is a color singlet and ensure that the respective evolution equation is free of infrared problems [63].

The second line in eq. (3.23) follows after reexpressing the adjoint Wilson line in terms of fundamental ones, according to $U_{\boldsymbol{z}}^{\dagger a b} t^{b}=V_{\boldsymbol{z}} t^{a} V_{\boldsymbol{z}}^{\dagger}$, and then using the Fierz identity

$$
\operatorname{tr}\left(t^{a} A t^{a} B\right)=\frac{1}{2} \operatorname{tr} A \operatorname{tr} B-\frac{1}{2 N_{c}} \operatorname{tr}(A B)
$$

By adding together the above results, one sees that the terms proportional to $1 / N_{c}^{2}$ exactly cancel between 'crossing' and 'non-crossing' contributions, ${ }^{8}$ so the net result reads

$$
\frac{\partial\left\langle\hat{S}_{\boldsymbol{x} \boldsymbol{y}}\right\rangle_{Y}}{\partial Y}=\frac{\bar{\alpha}}{2 \pi} \int_{\boldsymbol{z}} \mathcal{M}_{\boldsymbol{x} \boldsymbol{y} \boldsymbol{z}}\left\langle\hat{S}_{\boldsymbol{x} \boldsymbol{z}} \hat{S}_{\boldsymbol{z} \boldsymbol{y}}-\hat{S}_{\boldsymbol{x} \boldsymbol{y}}\right\rangle_{Y}
$$

where we have also taken the average over the target. Formally, this equation depicts the evolution as the splitting of the original dipole $(\boldsymbol{x}, \boldsymbol{y})$ into a system of two dipoles, $(\boldsymbol{x}, \boldsymbol{z})$ and $(\boldsymbol{z}, \boldsymbol{y})$, which have a common leg at $\boldsymbol{z}$. This would be the actual physical picture at large $N_{c}$, but it formally holds also for finite $N_{c}$, due to the 'accidental' cancellation of the terms suppressed by $1 / N_{c}^{2}$.

In deriving eq. (3.26) as above, it has been convenient to work in a frame where the projectile was a bare dipole prior to the evolution step under consideration. By boost invariance, the ensuing equation is valid in any frame (so long as the projectile remains dilute, of course).

\footnotetext{
${ }^{8}$ This cancellation too can be recognized as a consequence of the identity (2.13).
} 
(ii) The manifestly probabilistic approach. In applying eq. (2.18) to a SW target, one must perform manipulations similar to those in section 3.1 - that is, distinguish between 'crossing' and 'non-crossing' contributions and then compute the respective time integrals. In doing that, it is essential to keep together the 'real' and 'virtual' pieces in eq. (2.18), for each of the three integration ranges. Then the calculations simplify since (a) there are no divergences in the intermediate stages, and (b) the full result comes from the 'crossing' pieces ('real' plus 'virtual') alone. Moreover, the associated manipulations have a clear probabilistic interpretation, in agreement with the discussion in section 2.2.

To demonstrate this, notice the following identities for the action of the functional derivative on the dipole $S$-matrix:

$$
\begin{aligned}
R_{\boldsymbol{r}_{1}}^{a} R_{\boldsymbol{r}_{2}}^{a} \hat{S}_{\boldsymbol{x} \boldsymbol{y}}=L_{\boldsymbol{r}_{1}}^{a} L_{\boldsymbol{r}_{2}}^{a} \hat{S}_{\boldsymbol{x} \boldsymbol{y}} & =\left[-g^{2} C_{F}\left(\delta_{\boldsymbol{r}_{1} \boldsymbol{x}}-\delta_{\boldsymbol{r}_{1} \boldsymbol{y}}\right)\left(\delta_{\boldsymbol{r}_{2} \boldsymbol{x}}-\delta_{\boldsymbol{r}_{2} \boldsymbol{y}}\right)\right] \hat{S}_{\boldsymbol{x} \boldsymbol{y}} \\
& =\left(\left.J^{a}\left(t_{2}, \boldsymbol{r}_{2}\right) J^{a}\left(t_{1}, \boldsymbol{r}_{1}\right) \hat{S}_{\boldsymbol{x} \boldsymbol{y}}\right|_{A^{-}=0}\right) \hat{S}_{\boldsymbol{x} \boldsymbol{y}},
\end{aligned}
$$

where the equality in the second line holds for any $t_{1}$ and $t_{2}$. These identities imply that the 'virtual' and 'real' contributions mutually cancel within the 'non-crossing' terms, as anticipated.

Consider now the respective 'crossing' contributions. For the 'real' term, this has been already computed in eq. (3.23). For the 'virtual' term, we also need the following integral

$$
\int_{-\infty}^{0} \mathrm{~d} t_{1} \int_{0}^{\infty} \mathrm{d} t_{2} \partial_{\boldsymbol{r}_{1}}^{i} \partial_{\boldsymbol{r}_{2}}^{i} G_{0}\left(t_{2}-t_{1}, \boldsymbol{r}_{2}-\boldsymbol{r}_{1} ; p^{+}\right)=-\frac{2}{p^{+}} \int \frac{\mathrm{d}^{2} \boldsymbol{p}}{(2 \pi)^{2}} \frac{\mathrm{e}^{\mathrm{i} \boldsymbol{p} \cdot\left(\boldsymbol{r}_{2}-\boldsymbol{r}_{1}\right)}}{p_{\perp}^{2}} .
$$

By using this and eqs. (3.27), (3.15), and (3.24), one finds the 'virtual-crossing' contribution:

$$
-\left(\left.\Delta H_{\mathrm{rad}} \hat{S}_{\boldsymbol{x} \boldsymbol{y}}\right|_{A^{-}=0}\right) \hat{S}_{\boldsymbol{x} \boldsymbol{y}}=-\frac{\alpha_{s} C_{F}}{\pi^{2}} \int_{\boldsymbol{z}} \mathcal{M}_{\boldsymbol{x} \boldsymbol{y} \boldsymbol{z}} \hat{S}_{\boldsymbol{x} \boldsymbol{y}}
$$

This is the same as the contribution (3.22) of the 'non-crossing' terms in the JIMWLK Hamiltonian. By adding this to the 'real-crossing' piece in eq. (3.23), one finally recovers the BK equation (3.26).

The probabilistic interpretation is now manifest. The quantity $(\bar{\alpha} / 2 \pi) \mathcal{M}_{\boldsymbol{x} \boldsymbol{y} \boldsymbol{z}} \mathrm{d} Y \mathrm{~d}^{2} \boldsymbol{z}$ is the differential probability for emitting a gluon at transverse coordinate $\boldsymbol{z}$ out of the quarkantiquark dipole $(\boldsymbol{x}, \boldsymbol{y})$. The 'real-crossing' piece represents the process where the evolved partonic system (quark, antiquark, and gluon) exists at the time of scattering $x^{+}=0$. The 'virtual-crossing' piece measures the decrease in the probability to find the original $q \bar{q}$ dipole at $x^{+}=0$. This decrease is associated with evolution processes which occur either before $\left(x^{+}<0\right)$, or after $\left(x^{+}>0\right)$, the scattering. So, the 'virtual-crossing' contribution must be equal to that of such genuinely 'non-crossing' processes. This is indeed what we have found in eq. (3.29).

The validity of this interpretation is also comforted by the fact the 'real' and 'virtual' terms in the BK equation separately develop logarithmic 'ultraviolet' divergences, which precisely cancel in their sum. These divergences, coming from the poles of the dipole kernel at $\boldsymbol{z}=\boldsymbol{x}$ and $\boldsymbol{z}=\boldsymbol{y}$, correspond to self-energy corrections where the gluon lies arbitrarily 
close to its parent quark in the transverse plane. But such short-distance emissions should not affect the $S$-matrix since the scattering cannot distinguish between a bare quark and a bare quark accompanied by its radiation gluon, so long as the two partons are very close to each other. And indeed, by inspection of (3.26) one sees that the pole of $\mathcal{M}_{\boldsymbol{x} \boldsymbol{y} \boldsymbol{z}}$ at, say, $\boldsymbol{z}=\boldsymbol{x}$ is compensated by the linear combination of Wilson line correlators, due to 'color transparency' $\left(\hat{S}_{\boldsymbol{x} \boldsymbol{z}} \rightarrow 1\right.$ as $\left.\boldsymbol{z} \rightarrow \boldsymbol{x}\right)$.

Notice that, in order for such cancellations to work, it has been essential to have the right relative coefficient between the 'virtual' term and the 'real' one (or, equivalently, between 'crossing' and 'non-crossing' contributions). In turn, this emphasizes the importance of using the adiabatic prescription when computing the time integrals in section 3.1 (cf. the discussion after eq. (3.12)).

For later reference, it is useful to exhibit the limit of eq. (3.26) in the regime where the scattering is weak, which is the celebrated BFKL equation [64-66]. This is obtained by linearizing the BK equation (3.26) w.r.t. the dipole amplitude $\mathcal{T}_{Y}(\boldsymbol{x}, \boldsymbol{y}) \equiv 1-\left\langle\hat{S}_{\boldsymbol{x} \boldsymbol{y}}\right\rangle_{Y}$ and reads

$$
\frac{\partial \mathcal{T}_{Y}(\boldsymbol{x}, \boldsymbol{y})}{\partial Y}=\frac{\bar{\alpha}}{2 \pi} \int_{\boldsymbol{z}} \mathcal{M}_{\boldsymbol{x} \boldsymbol{y} \boldsymbol{z}}\left\{\mathcal{T}_{Y}(\boldsymbol{x}, \boldsymbol{z})+\mathcal{T}_{Y}(\boldsymbol{z}, \boldsymbol{y})-\mathcal{T}_{Y}(\boldsymbol{x}, \boldsymbol{y})\right\}
$$

This equation is valid so long as $\mathcal{T}_{Y} \ll 1$ and describes the high-energy evolution of the amplitude for single scattering. It is also interesting to consider the 'infrared' (large $\boldsymbol{z}$ ) behavior of the integral above. As already noticed, one has $\mathcal{M}_{\boldsymbol{x} \boldsymbol{y z}} \sim 1 / z_{\perp}^{4}$ when $z_{\perp} \rightarrow \infty$. Also, to leading order in pQCD, the dipole amplitude behaves like $\mathcal{T}_{0}(\boldsymbol{r}) \propto r^{2} \ln r^{2}$, hence for large $z_{\perp}$ one can write $\mathcal{T}_{0}(\boldsymbol{x}, \boldsymbol{z}) \simeq \mathcal{T}_{0}(\boldsymbol{z}, \boldsymbol{y}) \sim z_{\perp}^{2}$. With this behavior, the integral over $\boldsymbol{z}$ in eq. (3.30) would be logarithmically divergent. This is the familiar 'collinear' divergence of bremsstrahlung in QCD. One may (legitimately) question the validity of this linear approximation in the limit where $z_{\perp}$ is large. But as a matter of facts, the solution to the BFKL equation with $\mathcal{T}_{0}$ as an initial condition is known to be infrared safe: the successive iterations of this equation introduce an 'anomalous dimension', that is, they modify the dominant behavior of the amplitude at small $r$ according to $r^{2} \rightarrow r^{2 \gamma}$, where $\gamma<1$ depends upon the direction of evolution in the $\left(Y, \ln r^{2}\right)$ plane (see e.g. [18] for details). For any such a $\gamma$, the integral eq. (3.30) is indeed convergent at large $z_{\perp}$. This is interesting to keep in mind in view of the comparison with the corresponding approximation for an extended target (a medium), to be discussed in section 4.3.

Note finally that eq. (3.26) is not a closed equation - its r.h.s. also involves the $S$ matrix $\left\langle\hat{S}_{\boldsymbol{x} \boldsymbol{z}} \hat{S}_{\boldsymbol{z} \boldsymbol{y}}\right\rangle_{Y}$ for a system of two dipoles - , so it cannot be solved as it stands. This is truly the first equation from an infinite hierarchy, the B-JIMWLK hierarchy, which describes the coupled evolution of scattering amplitudes for dilute systems with increasing complexity in terms of partonic structure. This hierarchy simplifies in the large $N_{c}$ limit, where expectation values of gauge invariant operators can be factorized from each other. In that limit, eq. (3.26) reduces to a closed equation for the dipole $S$-matrix, originally derived by Kovchegov [20]:

$$
\frac{\partial\left\langle\hat{S}_{\boldsymbol{x} \boldsymbol{y}}\right\rangle_{Y}}{\partial Y}=\frac{\bar{\alpha}}{2 \pi} \int_{\boldsymbol{z}} \mathcal{M}_{\boldsymbol{x} \boldsymbol{y} \boldsymbol{z}}\left\{\left\langle\hat{S}_{\boldsymbol{x} \boldsymbol{z}}\right\rangle_{Y}\left\langle\hat{S}_{\boldsymbol{z} \boldsymbol{y}}\right\rangle_{Y}-\left\langle\hat{S}_{\boldsymbol{x} \boldsymbol{y}}\right\rangle_{Y}\right\}
$$

In the next section, we shall generalize this equation to the case of an extended target. 


\section{The high-energy evolution of transverse momentum broadening}

Starting with this section, we address the main physical problem of interest for us here, namely the high energy evolution of a 'hard probe' (energetic parton) which crosses a dense QCD medium, like a weakly-coupled quark-gluon plasma (QGP). The main difference with respect to the 'shockwave' problem that we considered in section 3 refers to the center-ofmass energy of the process: for the problem of jet quenching, this energy is considerably smaller. To be specific, consider the scattering between the hard probe and the medium in the target rest frame. In section 3 , we have assumed that the energy $E \equiv p_{0}^{+}$of the incoming projectile is much higher than the characteristic energy scale of the target, $\omega_{c}=Q_{s}^{2} L$. This condition ensured the existence of a large energy phase-space, at $\omega_{c} \ll p^{+} \ll E$, for gluon fluctuations with very large lifetimes $\tau \gg L$, to which the target effectively looks like a shockwave. But in the problem of jet quenching, these scales $E$ and $\omega_{c}$ are comparable with each other. For instance, for jet production in $A A$ collisions at the LHC, the typical jet energies are of the order of $100 \mathrm{GeV}$, whereas the medium scale $\omega_{c}$ (which in this context is most naturally evaluated as $\omega_{c}=\hat{q} L^{2}$, with $\hat{q}$ the jet quenching parameter) is in the ballpark of $50 \mathrm{GeV}$. Accordingly, the (soft) gluon fluctuations of the projectile have typical energies $p^{+} \ll \omega_{c}$ and lifetimes much smaller than $L$. As generally for bremsstrahlung, these soft fluctuations are favored by the phase-space, leading to relatively large quantum corrections, enhanced by the energy logarithm $\ln (L / \lambda)$. This is an 'energy' logarithm, since it comes from the longitudinal phase-space for the fluctuations: their lifetimes $\tau$ are constrained according to $\lambda<\tau<L$, with $\lambda$ the wavelength of a typical medium constituent. (Alternatively, this logarithm could be rewritten as $\ln \left(\omega_{c} / \omega_{0}\right)$ with $\omega_{0} \equiv \hat{q} \lambda^{2}$.) The resummation of these large radiative corrections is the scope of the high-energy evolution, which in this context is most conveniently described as an evolution with increasing the medium size $L$ (the upper limit on the longitudinal phase-space for fluctuations).

This evolution is similar to the Balitsky-JIMWLK evolution discussed in section 3 in that it is non-linear: it deals with soft gluon emissions in the presence of a strong background field, so the effects of multiple scattering must be resumed to all orders. But unlike in the case of a shockwave, the multiple scattering inside the extended medium cannot be treated in the eikonal approximation. This is a source of several 'technical' complications, that we here anticipate.

First the gluon propagator in the background of the target field is known only as a formal path integral, cf. eq. (2.10). Accordingly, the Wilson lines attached to the gluon fluctuations become functionals of the gluon trajectories, which are themselves random.

Second, the time integrations in eq. (2.4) cannot be performed directly at the level of the Hamiltonian, i.e. before acting with $\Delta H$ on the scattering operator representing the projectile. This can be understood by inspection of eq. (2.6): if the time argument $t$ of the functional derivative $J^{a}(t, \boldsymbol{r})$ lies inside the medium, then the Wilson lines in the r.h.s. of eq. (2.6) are explicitly time-dependent, in contrast to what happened for a shockwave (compare to eqs. (3.3)-(3.4)). So one cannot disentangle the integrations over the emission times $t_{1}$ and $t_{2}$ from the Wilson line correlations. The precise structure of the latter depends of course upon the nature of the projectile. 
Third, even after choosing a projectile and using $\Delta H$ to construct the associated evolution equation, this equation is still too complicated to be dealt with in full generality. Not only this is not a closed equation (a feature that we are already familiar with from the example of the B-JIMWLK hierarchy), but this equation is also functional (the unknown correlators enter under the path integral representing the trajectory of the fluctuation) and non-local in time (the correlators depend upon the integration variables $t_{1}$ and $t_{2}$ ). The fact that one cannot perform the time integrations in general (i.e. without additional simplifications) also implies that we shall not be able to demonstrate the logarithmic enhancement of the radiative corrections directly at the level of the equations. This enhancement will become manifest only after performing additional approximations, which refer to limiting cases of special interest and lead to more tractable equations.

For simplicity, we shall focus on the evolution of a color dipole. This is pertinent indeed, since the corresponding scattering amplitude enters the calculation of two important observables - the transverse momentum broadening and the energy loss by an energetic parton - that we shall discuss in this and the next coming sections. Also, we shall use a general set-up which is similar to that in the previous section: the quark is approaching the medium from very far away and its interactions are adiabatically switched off at large times, $\left|x^{+}\right| \rightarrow \infty$. This is not necessarily the actual situation in a nucleus-nucleus collision, where the quark can also be created inside the medium, via some hard process. This would lead to additional radiation which could mix with the quantum fluctuations triggered by the interactions in the medium. The simplest way to avoid such a mixing is to assume that the quark was on-shell before it enters the medium, as we shall actually do.

\subsection{The tree-level approximation}

In preparation for the quantum evolution to be discussed in the next sections, we shall first briefly review the tree-level calculation of the transverse momentum broadening. This will give us the opportunity to introduce the relevant scales and notations and, moreover, it will inspire some of the approximations to be performed later on.

At tree-level, the problem of transverse momentum broadening for an energetic quark which enters the medium is formally similar to that of quark production in $p A$ collisions at forward rapidities. In both cases, the transverse-momentum distribution $\mathrm{d} N / \mathrm{d}^{2} \boldsymbol{p}$ of the quark after the collision can be computed, within the limits of the eikonal approximation, as the Fourier transform of a dipole forward amplitude (below, $\boldsymbol{r} \equiv \boldsymbol{x}-\boldsymbol{y}$ ):

$$
\frac{\mathrm{d} N}{\mathrm{~d}^{2} \boldsymbol{p}}=\frac{1}{(2 \pi)^{2}} \int_{\boldsymbol{r}} \mathrm{e}^{-\mathrm{i} \boldsymbol{p} \cdot \boldsymbol{r}}\left\langle\hat{S}_{\boldsymbol{x} \boldsymbol{y}}\right\rangle .
$$

The 'dipole' here is merely a mathematical construction: the 'quark leg at $\boldsymbol{x}$ ' represents the physical quark in the direct amplitude, whereas the 'antiquark leg at $\boldsymbol{y}$ ' is the physical quark in the complex conjugate amplitude. As usual, the brackets within $\left\langle\hat{S}_{\boldsymbol{x} y}\right\rangle$ denotes the target average over the configurations of the color field $A_{a}^{-}(x)$. The target is a weaklycoupled QCD medium with longitudinal support at $0<x^{+}<L$. For simplicity, we assume this medium to be homogeneous (on the average) in the transverse plane. Accordingly, the average $S$-matrix depends only upon the dipole size $\boldsymbol{r}=\boldsymbol{x}-\boldsymbol{y}$ and we can write 
$\left\langle\hat{S}_{\boldsymbol{x} \boldsymbol{y}}\right\rangle \equiv \mathcal{S}(\boldsymbol{r})$. Using $\mathcal{S}(0)=1$ ('color transparency'), one sees that the distribution (4.1) is properly normalized: $\int \mathrm{d}^{2} \boldsymbol{p}\left(\mathrm{d} N / \mathrm{d}^{2} \boldsymbol{p}\right)=1$.

A weakly-coupled medium, such as a QGP with sufficiently high temperature $T$, can be described as an incoherent collection of independent color charges, 'quarks' and 'gluons'. These charges will be assumed to be point-like and to have no other mutual interactions, except for those responsible for the screening of the color interactions over a (transverse) distance $r \sim 1 / m_{D}$, with $m_{D}$ the 'Debye mass'. Under these assumptions, the only nontrivial correlator of the target field $A^{-}$is the respective 2-point function, which has the following structure

$$
\left\langle A_{a}^{-}\left(x^{+}, x^{-}, \boldsymbol{x}\right) A_{b}^{-}\left(y^{+}, y^{-}, \boldsymbol{y}\right)\right\rangle_{0}=\delta_{a b} \delta\left(x^{+}-y^{+}\right) n\left(x^{+}\right) \gamma(\boldsymbol{x}-\boldsymbol{y}),
$$

where $n$ is the number density of the medium constituents (more precisely, a linear combination of the respective densities for quarks and gluons, weighted with appropriate color factors). As indicated in eq. (4.2), this density can generally depend upon $x^{+}$(e.g. for an expanding medium), but here we shall mostly work with a medium which is uniform in $x^{+}$ (within its longitudinal support at $0<x^{+}<L$, of course). Also

$$
\gamma(\boldsymbol{k}) \equiv \int \mathrm{d}^{2} \boldsymbol{r} \mathrm{e}^{\mathrm{i} \boldsymbol{k} \cdot \boldsymbol{r}} \gamma(\boldsymbol{r}) \simeq \frac{g^{2}}{\boldsymbol{k}^{4}}
$$

with the approximate equality holding for $k_{\perp} \gg m_{D}$, is the square of the 2-dimensional Coulomb propagator. It is understood that eq. (4.3) must be used with an infrared cutoff $k_{\perp} \simeq m_{D}$.

The correlator (4.2) is local in the color indices, by gauge symmetry. It is furthermore independent of the light-cone variables $x^{-}$and $y^{-}$, and it is local in $x^{+}$, because of the high energy kinematics. These properties can be best understood in a frame where the medium is a ultrarelativistic left mover: then, the dynamics in $x^{-}$(the light-cone 'time' for a left mover) is frozen by Lorentz time dilation, whereas the correlation length in $x^{+}$gets squeezed by Lorentz longitudinal contraction. The locality in $x^{+}$is clearly an idealization, whose limitations will be discussed in section 4.3.3. The shockwave counterpart of eq. (4.2) is the description of a large nucleus in the McLerran-Venugopalan (MV) model, which employs a Gaussian CGC weight function [67, 68].

For the Gaussian field distribution in eq. (4.2), it is a straightforward exercise to compute the average $S$-matrix for a quark-antiquark dipole. One finds

$$
\mathcal{S}_{0}(\boldsymbol{r})=\exp \left\{-g^{2} C_{F} \int_{0}^{L} \mathrm{~d} x^{+} n\left(x^{+}\right) \int \frac{\mathrm{d}^{2} \boldsymbol{k}}{(2 \pi)^{2}} \gamma(\boldsymbol{k})\left(1-\mathrm{e}^{\mathrm{i} \boldsymbol{k} \cdot \boldsymbol{r}}\right)\right\} .
$$

The quantity within the braces is (minus) the amplitude for a single scattering between the dipole and the medium. The fact that the multiple scattering series exponentiates reflects the lack of non-trivial medium correlations: successive collisions proceed independently from each other.

Using eq. (4.3), one sees that the integral over $\boldsymbol{k}$ in eq. (4.4) is logarithmically sensitive to the IR cutoff $m_{D}$. We shall be mostly interested in small dipole sizes $r \equiv|\boldsymbol{r}| \ll 1 / m_{D}$. 
Then, there is a large logarithmic phase-space, at $m_{D} \ll k_{\perp} \ll 1 / r$. To leading logarithmic accuracy, the integral can be evaluated by expanding the complex exponential $\mathrm{e}^{\mathrm{i} \boldsymbol{k} \cdot \boldsymbol{r}}$ to second order (the linear term vanishes after angular integration). One thus finds (with $n\left(x^{+}\right)=n_{0}$ from now on)

$$
\mathcal{S}_{0}(\boldsymbol{r}) \simeq \exp \left\{-\frac{1}{4} L \hat{q}\left(1 / r^{2}\right) \boldsymbol{r}^{2}\right\}
$$

where $\hat{q}$ is the jet quenching parameter for an incoming quark:

$$
\hat{q}\left(Q^{2}\right) \equiv g^{2} C_{F} n_{0} \int^{Q^{2}} \frac{\mathrm{d}^{2} \boldsymbol{k}}{(2 \pi)^{2}} \boldsymbol{k}^{2} \gamma(\boldsymbol{k}) \simeq 4 \pi \alpha_{s}^{2} C_{F} n_{0} \ln \frac{Q^{2}}{m_{D}^{2}} .
$$

In the above integral, the 'collision kernel' $g^{2} C_{F} \gamma(\boldsymbol{k})$ (the differential cross-section for the scattering between the quark and the medium) is weighted by the transverse momentum squared $k_{\perp}^{2}$ transferred in the collision. Accordingly, $\hat{q}\left(Q^{2}\right)$ is proportional to a transport cross-section - the total cross-section for collisions which are accompanied by hard transverse-momentum transfers, within the range $m_{D}^{2} \ll k_{\perp}^{2} \ll Q^{2}$. For a weakly coupled QGP, one has, parametrically, $n_{0} \sim N_{c} T^{3}, m_{D}^{2} \sim \alpha_{s} N_{c} T^{2}$, and hence $\hat{q} \sim \alpha_{s}^{2} N_{c}^{2} T^{3} \ln \left(1 / \alpha_{s} N_{c}\right)$. (See refs. [6, 7, 69] for detailed calculations.)

The dipole scattering becomes strong when the exponent in eq. (4.5) is of order one, or larger. This happens when $r \gtrsim 1 / Q_{s}$, with $Q_{s}$ a characteristic transverse momentum scale, defined as

$$
Q_{s}^{2}=L \hat{q}\left(Q_{s}^{2}\right)=4 \pi \alpha_{s}^{2} C_{F} n_{0} L \ln \frac{Q_{s}^{2}}{m_{D}^{2}}
$$

(We implicitly assume that $Q_{s}$ is much larger than $m_{D}$, which in turn requires the medium size $L$ to be large enough; see section 4.3 .3 for details.) This scale $Q_{s}$ is generally referred to as the 'target saturation momentum', because the physics responsible for the unitarization of the dipole amplitude - the multiple scattering between the dipole and the color charges in the target - can also be viewed, in a suitable frame where the target is highly boosted, as the result of non-linear phenomena in the gluon distribution in the target, leading to gluon saturation $[12,35,36]$. In section 4.4 , we shall argue that this profound relation between jet quenching and gluon saturation, which here has been observed at tree-level, is also preserved by the high-energy evolution.

Using eq. (4.5), one can now estimate the Fourier transform in eq. (4.1) for $p_{\perp} \gg m_{D}$. Consider first the case $p_{\perp} \lesssim Q_{s}$; then, the integral in eq. (4.1) is cut off by the dipole $S$ matrix at a value $r \simeq 1 / Q_{s}$. To the accuracy of interest, one can ignore the slow dependence of the jet quenching parameter upon $r$, and thus deduce

$$
\frac{\mathrm{d} N}{\mathrm{~d}^{2} \boldsymbol{p}} \simeq \frac{1}{\pi Q_{s}^{2}} \mathrm{e}^{-\boldsymbol{p}^{2} / Q_{s}^{2}}
$$

This Gaussian distribution is the hallmark of a diffusive process - a random walk in the transverse momentum space, leading to a momentum broadening $\left\langle p_{\perp}^{2}\right\rangle \simeq Q_{s}^{2}$ 一, which is induced by a succession of independent collisions in the medium. 
Consider also the high-momentum limit $p_{\perp} \gg Q_{s}$; then, the integral in eq. (4.1) is cut off by the complex exponential at a value $r \sim 1 / p_{\perp} \ll 1 / Q_{s}$, so it is appropriate to expand the dipole $S$-matrix to linear order in its exponent. This gives

$$
\begin{aligned}
\frac{\mathrm{d} N}{\mathrm{~d}^{2} \boldsymbol{p}} & \simeq \frac{\alpha_{s}^{2} C_{F}}{4 \pi} n_{0} L \int_{\boldsymbol{r}} \mathrm{e}^{-\mathrm{i} \boldsymbol{p} \cdot \boldsymbol{r}}\left(-r^{2}\right) \ln \frac{1}{r^{2} m_{D}^{2}}=\frac{4 \alpha_{s}^{2} C_{F} n_{0} L}{p_{\perp}^{4}} \\
& =\frac{1}{\pi Q_{s}^{2} \ln \left(Q_{s}^{2} / m_{D}^{2}\right)} \frac{Q_{s}^{4}}{p_{\perp}^{4}} .
\end{aligned}
$$

The logarithmic scale dependence of $\hat{q}\left(1 / r^{2}\right)$ has been essential in deriving this result. As clear from its above derivation, the $1 / p_{\perp}^{4}$ tail in the spectrum at high $p_{\perp}$ is produced via a single, hard, scattering. This represents a rather rare event, as visible from the fact that the integral of (4.9) over $p_{\perp}>Q_{s}$ is suppressed by a large logarithm:

$$
\int_{Q_{s}} \mathrm{~d}^{2} \boldsymbol{p} \frac{\mathrm{d} N}{\mathrm{~d}^{2} \boldsymbol{p}} \simeq \frac{1}{\ln \left(Q_{s}^{2} / m_{D}^{2}\right)} \ll 1
$$

This is furthermore in agreement with the fact that the probability sum rule $\int \mathrm{d}^{2} \boldsymbol{p}\left(\mathrm{d} N / \mathrm{d}^{2} \boldsymbol{p}\right)=1$ is already exhausted (to the leading-logarithmic accuracy of interest) by the contribution (4.8) of relatively soft $\left(k_{\perp} \lesssim Q_{s}\right)$ multiple scattering.

In what follows, we shall be mostly interested in typical events, in which the final spectrum is the result of multiple soft scattering and has the Gaussian form in eq. (4.8). Accordingly, we shall focus on a quark-antiquark dipole with transverse size $r \sim 1 / Q_{s}$. This in turn implies that the exponent in eq. (4.5) for the dipole $S$-matrix is of $\mathcal{O}(1)$ : on the average, the dipole undergoes one inelastic scattering while crossing the medium. This more precisely means that the dipole may undergo a single hard collision, with a transferred momentum $k_{\perp} \sim Q_{s}$, or a large number of softer collisions, but in such a way that the total transferred momentum squared is again of order $Q_{s}^{2}$. The present calculation cannot distinguish between such scenarios, so in that sense the jet quenching parameter $\hat{q}\left(Q_{s}^{2}\right)$ is not really a local transport coefficient, but rather a measure of the average properties of the medium coarse-grained over a longitudinal distance of order $L$. (This is also visible in the fact that the quantity $\hat{q}\left(Q_{s}^{2}\right)$ 'knows' about the overall size $L$ of the medium, via its logarithmic dependence upon $Q_{s}^{2} \propto L$.) This should be kept in mind when interpreting the radiative corrections to be computed in what follows.

\subsection{The dipole evolution equation}

In this section, we shall construct a generalization of the Balitsky-Kovchegov equation that is, a non-linear equation for the high energy evolution of the dipole $S$-matrix - to the case where the dense target is an extended medium. Then, by using this evolution equation together with eq. (4.1), we shall be able to study the high-energy evolution of the transverse momentum broadening and related phenomena. At this point it is important to notice that the relation (4.1) between the cross-section for $p_{\perp}$-broadening and the forward dipole amplitude is known to be preserved by the high-energy evolution to next-to-leading logarithmic accuracy, at least $[13,70]$. Here, we shall merely work at leading logarithmic accuracy, so we can indeed rely on this dipole picture for the present purposes. 
As in section 3.2, we shall construct an evolution equation for the average dipole $S$ matrix by first acting with the evolution Hamiltonian $\Delta H$ on the dipole operator $\hat{S}_{x y}$ and then taking the average over the target. This procedure implicitly assumes that the projectile is a 'bare' dipole prior to the evolution step under consideration, a condition which can always be satisfied by appropriately choosing the Lorentz frame: namely, we view this particular step of the evolution in a frame where the dipole is relatively slow, but the medium is highly boosted. This in turn implies that the effects of the earlier steps in the evolution have been incorporated in the distribution of the color fields in the target. So, in general, this distribution can be more complicated than the Gaussian introduced in the previous subsection, cf. eq. (4.2), and which applies at tree-level.

In the present context, it is more advantageous to use the alternative form (2.18) for the action of $\Delta H$. In this form, the 'virtual' term required by probability conservation is already built in. This allows one to avoid spurious divergences already before integrating over the emission times $t_{1}$ and $t_{2}$ (an operation that we shall not be able to perform in full generality). We start by computing the coefficient of this 'virtual' term, which is independent of the background field and hence particularly simple. Using eqs. (2.20) and (3.27), one finds

$$
\begin{aligned}
- & \left.\Delta H_{\mathrm{rad}} \hat{S}_{\boldsymbol{x} \boldsymbol{y}}\right|_{A^{-}=0} \\
& =\left.\left.\frac{g^{2} C_{F}}{2 \pi} \int_{\omega}^{\omega_{c}} \frac{\mathrm{d} p^{+}}{\left(p^{+}\right)^{2}} \int_{-\infty}^{\infty} \mathrm{d} t_{2} \int_{-\infty}^{t_{2}} \mathrm{~d} t_{1}\left[\partial_{\boldsymbol{r}_{2}}^{i} \partial_{\boldsymbol{r}_{1}}^{i} G_{0}\left(t_{2}-t_{1}, \boldsymbol{r}_{2}-\boldsymbol{r}_{1} ; p^{+}\right)\right]\right|_{\boldsymbol{r}_{2}=\boldsymbol{y}} ^{\boldsymbol{r}_{2}=\boldsymbol{x}}\right|_{\boldsymbol{r}_{1}=\boldsymbol{y}} ^{\boldsymbol{r}_{1}=\boldsymbol{x}} .
\end{aligned}
$$

It would be straightforward to perform the remaining integrations in the expression above, but its present form is more useful for what follows.

As compared to eq. (2.4), in writing eq. (4.11) we have adapted the integration limits for $p^{+}$to the problem at hand. Namely, the upper limit is the characteristic medium scale (cf. eq. (3.1))

$$
\omega_{c}=Q_{s}^{2} L=\hat{q}\left(Q_{s}^{2}\right) L^{2},
$$

which sets the upper cutoff on the energy of the gluon fluctuations (indeed, fluctuations with larger energies $\omega>\omega_{c}$ would have a formation time larger than $L$ ). The lower limit $\omega$ is the minimal energy allowed for a gluon fluctuation. Eventually this will be identified with another medium scale $\omega_{0} \equiv \hat{q} \lambda^{2}$, corresponding to a minimal formation time equal to $\lambda$ (see the discussion in section 4.3.3). But in the interesting situation where $L \gg \lambda$, we expect large radiative corrections enhanced by the $\operatorname{logarithm} \ln \left(\omega_{c} / \omega_{0}\right)=\ln (L / \lambda)$, hence we need to 'evolve' from $\omega \sim \omega_{c}$ down to $\omega=\omega_{0}$ in a differential way. For the time being, it is convenient to choose this lower limit $\omega$ as the parameter for the evolution.

Consider now the 'real' term in eq. (2.18), which involves the radiation piece of the background field propagator, cf. eq. (2.19). By repeatedly using eq. (2.6), one finds

$$
\begin{gathered}
\Delta H_{\mathrm{rad}} \hat{S}_{\boldsymbol{x} \boldsymbol{y}}=-g^{2} \int_{\omega}^{\omega_{c}} \frac{\mathrm{d} p^{+}}{2 \pi} \frac{1}{\left(p^{+}\right)^{2}} \int_{-\infty}^{\infty} \mathrm{d} t_{2} \int_{-\infty}^{t_{2}} \mathrm{~d} t_{1} \partial_{\boldsymbol{r}_{1}}^{i} \partial_{\boldsymbol{r}_{2}}^{i} G_{a b}\left(t_{2}, \boldsymbol{r}_{2} ; t_{1}, \boldsymbol{r}_{1} ; p^{+}\right) \\
\left\{\delta_{\boldsymbol{r}_{1} \boldsymbol{x}} \delta_{\boldsymbol{r}_{2} \boldsymbol{x}} \frac{\operatorname{tr}}{N_{c}}\left[\left(V_{\infty, t_{2}}^{\dagger} t^{a} V_{t_{2} t_{1}}^{\dagger} t^{b} V_{t_{1},-\infty}^{\dagger}\right)_{\boldsymbol{x}} V_{\boldsymbol{y}}\right]\right.
\end{gathered}
$$



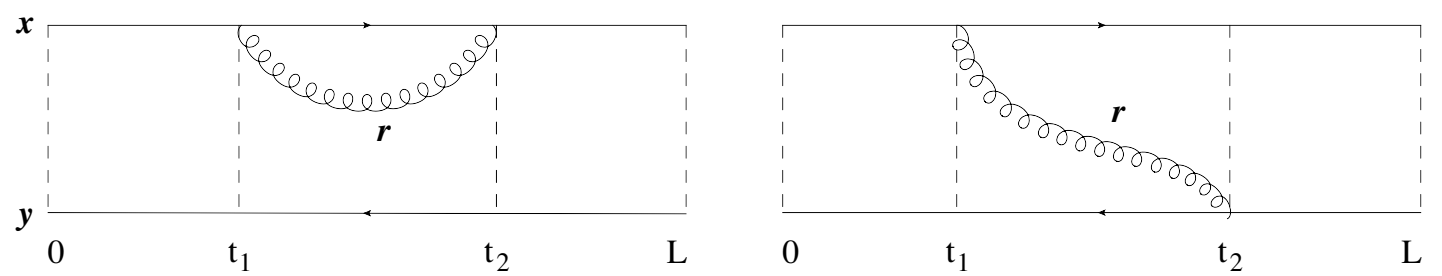

Figure 1. Two diagrams illustrating the dipole evolution described by eq. (4.13) (they correspond to the first and respectively the fourth term in the r.h.s. of eq. (4.13)). It is understood that all the partonic lines (quark, antiquark, and gluon) are accompanied by Wilson lines describing scattering off the medium.

$$
\begin{aligned}
& +\delta_{\boldsymbol{r}_{1} \boldsymbol{y}} \delta_{\boldsymbol{r}_{2} \boldsymbol{y}} \frac{\operatorname{tr}}{N_{c}}\left[V_{\boldsymbol{x}}^{\dagger}\left(V_{t_{1},-\infty} t^{b} V_{t_{2} t_{1}} t^{a} V_{\infty, t_{2}}\right)_{\boldsymbol{y}}\right] \\
& -\delta_{\boldsymbol{r}_{1} \boldsymbol{y}} \delta_{\boldsymbol{r}_{2} \boldsymbol{x}} \frac{\operatorname{tr}}{N_{c}}\left[\left(V_{\infty, t_{2}}^{\dagger} t^{a} V_{t_{2},-\infty}^{\dagger}\right)_{\boldsymbol{x}}\left(V_{t_{1},-\infty} t^{b} V_{\infty, t_{1}}\right)_{\boldsymbol{y}}\right] \\
& \left.-\delta_{\boldsymbol{r}_{1} \boldsymbol{x}} \delta_{\boldsymbol{r}_{2} \boldsymbol{y}} \frac{\operatorname{tr}}{N_{c}}\left[\left(V_{\infty, t_{1}}^{\dagger} t^{b} V_{t_{1},-\infty}^{\dagger}\right)_{\boldsymbol{x}}\left(V_{t_{2},-\infty} t^{a} V_{\infty, t_{2}}\right)_{\boldsymbol{y}}\right]\right\} .
\end{aligned}
$$

Note that $V_{t_{2} t_{1}} \equiv\left(V_{t_{2} t_{1}}^{\dagger}\right)^{\dagger}$ truly describes backward propagation in time, from $t_{2}$ to $t_{1}$ (recall that $t_{2}>t_{1}$ ). The physical interpretation of the four terms within the braces in the r.h.s. of eq. (4.13) is quite transparent (see also figure 1): the first two terms describes processes in which the soft gluon is emitted and then reabsorbed by a same leg of the dipole (either the quark, or the antiquark); the last two terms, which have the opposite sign, correspond to exchange processes where the gluon is emitted by the quark and then absorbed by the antiquark, or vice-versa.

To obtain an evolution equation, one needs to average eq. (4.13) over the target field distribution and also perform the integrations over the emission times $t_{1}$ and $t_{2}$. (Note that the target average should also include the adjoint Wilson line implicit in the structure of the gluon propagator, cf. eq. (2.10).) We here meet with one of the difficulties anticipated at the beginning of this section: unlike in the corresponding discussion for a shockwave, the support of the Wilson lines in eq. (4.13) is now truly dependent upon the emission times $t_{1}$ and $t_{2}$. Accordingly, the time integrations cannot be disentangled from the target correlations anymore: the Wilson line correlators which enter $\left\langle\Delta H \hat{S}_{\boldsymbol{x} \boldsymbol{y}}\right\rangle$ are explicitly time dependent. So, it seems impossible to make further progress in full generality - i.e., without additional assumptions about the medium correlations.

Inspired by the situation at tree-level and also by the mean field approximation to the B-JIMWLK equations [35, 37-43], which appears to be remarkably successful [43, 44], we shall from now on assume that the background field distribution remains approximatively Gaussian after including the effects of the high energy evolution. That is, the only nontrivial background field correlator is the respective 2-point function, which has the general structure (compare to eq. (4.2))

$$
\left\langle A_{a}^{-}\left(x^{+}, x^{-}, \boldsymbol{x}\right) A_{b}^{-}\left(y^{+}, y^{-}, \boldsymbol{y}\right)\right\rangle_{\omega}=\delta_{a b} \delta\left(x^{+}-y^{+}\right) \bar{\Gamma}_{\omega}\left(x^{+}, \boldsymbol{x}-\boldsymbol{y}\right)
$$


This depends upon the energy scale $\omega$ down to which one has integrated out the soft gluons, since this fixes the longitudinal resolution on which one probes the medium correlations. The $x^{+}$-dependence of the correlator $\bar{\Gamma}_{\omega}\left(x^{+}, \boldsymbol{x}-\boldsymbol{y}\right)$ reflects the evolution of the time inhomogeneity introduced at tree-level by the charged particles density $n\left(x^{+}\right)$, cf. eq. (4.2). Vice-versa, if the latter is independent of time, $n\left(x^{+}\right)=n_{0}$, then so is also the function $\bar{\Gamma}_{\omega}$ - except, of course, for the fact that its longitudinal support is restricted ${ }^{9}$ to $0<x^{+}<L$.

The main characteristic of the 2-point function (4.14) is to be local in time. This is well justified at tree-level and remains true in the presence of the high-energy evolution, because of the strong separation in longitudinal scales inherent in this evolution. To understand that, recall that we work in a frame where the target is a rapid left mover, hence the medium correlations are potentially modified by radiative corrections associated with the emission of (relatively) soft gluons by the medium constituents. Such 'soft' emissions carry longitudinal momenta $p^{-}$which are small compared to the respective momenta of their parent partons (the medium constituents), but much larger than the $p^{-}$component of any parton from the projectile (the original dipole or its gluon fluctuations). Accordingly, the scale for non-locality in $x^{+}$as induced by this evolution, namely $\Delta x^{+} \propto 1 / p^{-}$, is much smaller than the respective resolution scale (the lifetime $\tau \sim t_{2}-t_{1}$ ) of the gluon fluctuations of the projectile. ${ }^{10}$ To the latter, the medium correlations look still local, as at tree-level.

The locality of eq. (4.14) in $x^{+}$allows one to factorize the Wilson correlations within $\left\langle\Delta H \hat{S}_{x y}\right\rangle$ according to their time arguments. To be specific, consider the first among the four terms within the braces in eq. (4.13). After also including the adjoint Wilson line from the gluon propagator, cf. eq. (2.10), we are led to the following medium correlation function:

$$
\begin{aligned}
& \left\langle U_{t_{2} t_{1}}^{\dagger a b}[\boldsymbol{r}] \frac{\operatorname{tr}}{N_{c}}\left[\left(V_{\infty, t_{2}}^{\dagger} t^{a} V_{t_{2} t_{1}}^{\dagger} t^{b} V_{t_{1},-\infty}^{\dagger}\right)_{\boldsymbol{x}} V_{\boldsymbol{y}}\right]\right\rangle= \\
& =\left\langle\frac{\operatorname{tr}}{N_{c}}\left(V_{\infty, t_{2}}^{\dagger}(\boldsymbol{x}) V_{\infty, t_{2}}(\boldsymbol{y})\right)\right\rangle\left\langle U_{t_{2} t_{1}}^{\dagger a b}[\boldsymbol{r}] \frac{\operatorname{tr}}{N_{c}}\left(t^{a} V_{t_{2} t_{1}}^{\dagger}(\boldsymbol{x}) t^{b} V_{t_{2} t_{1}}(\boldsymbol{y})\right)\right\rangle \times \\
& \quad \times\left\langle\frac{\operatorname{tr}}{N_{c}}\left(V_{t_{1},-\infty}^{\dagger}(\boldsymbol{x}) V_{t_{1},-\infty}(\boldsymbol{y})\right)\right\rangle \\
& =\frac{N_{c}}{2}\left\{\left\langle\hat{S}_{\infty, t_{2}}(\boldsymbol{x}, \boldsymbol{y})\right\rangle\left\langle\hat{S}_{t_{2}, t_{1}}(\boldsymbol{x}, \boldsymbol{r}) \hat{S}_{t_{2}, t_{1}}(\boldsymbol{r}, \boldsymbol{y})\right\rangle\left\langle\hat{S}_{t_{1},-\infty}(\boldsymbol{x}, \boldsymbol{y})\right\rangle-\frac{1}{N_{c}^{2}}\langle\hat{S}(\boldsymbol{x}, \boldsymbol{y})\rangle\right\},
\end{aligned}
$$

where the first equality is obtained by using the Gaussian Ansatz (4.14) for the medium averages and the second one follows via the Fierz identity (3.25). We have defined the time-dependent dipole operator $\hat{S}_{t_{2}, t_{1}}(\boldsymbol{x}, \boldsymbol{y})$ via the obvious generalization of eq. (2.2). To simplify writing, we keep implicit the dependence of the various correlations upon the

\footnotetext{
${ }^{9}$ This restriction is not affected by the evolution since one can neglect the fluctuations occurring near the edges of the medium; see the discussion below.

${ }^{10}$ To fully justify the local approximation in eq. (4.14), one should also compare the non-locality scale $\Delta x^{+}$to the average time interval between two inelastic collisions in the medium. However it turns out that, for the gluon fluctuations of a dipole, this 'mean free path' is at least as large as the lifetime $\Delta t=t_{2}-t_{1}$ of the fluctuation, so this comparison introduces no additional restriction; see the discussion in sections 4.3.1 and 4.5 .
} 
renormalization scale $\omega$. Also, in the very last term we have reconstructed the average of the global $S$-matrix according to

$$
\langle\hat{S}(\boldsymbol{x}, \boldsymbol{y})\rangle=\left\langle\hat{S}_{\infty, t_{2}}(\boldsymbol{x}, \boldsymbol{y})\right\rangle\left\langle\hat{S}_{t_{2}, t_{1}}(\boldsymbol{x}, \boldsymbol{y})\right\rangle\left\langle\hat{S}_{t_{1},-\infty}(\boldsymbol{x}, \boldsymbol{y})\right\rangle .
$$

This last term, which is explicitly suppressed at large $N_{c}$, vanishes against the respective contribution of the 'virtual' term. A similar cancellation has been noticed in section 3.2 at the level of the BK equation. As in that case though, it is nevertheless convenient to consider the large- $N_{c}$ limit, which allows us to factorize the two-dipoles $S$-matrix during the lifetime of the fluctuation:

$$
\left\langle\hat{S}_{t_{2}, t_{1}}(\boldsymbol{x}, \boldsymbol{r}) \hat{S}_{t_{2}, t_{1}}(\boldsymbol{r}, \boldsymbol{y})\right\rangle \simeq\left\langle\hat{S}_{t_{2}, t_{1}}(\boldsymbol{x}, \boldsymbol{r})\right\rangle\left\langle\hat{S}_{t_{2}, t_{1}}(\boldsymbol{r}, \boldsymbol{y})\right\rangle \quad \text { at large } N_{c}
$$

One should keep in mind that $\hat{S}_{t_{2}, t_{1}}(\boldsymbol{x}, \boldsymbol{r}) \equiv \hat{S}_{t_{2}, t_{1}}(\boldsymbol{x},[\boldsymbol{r}(t)] ; \omega)$ is truly a functional of the path $\boldsymbol{r}(t)$, with $t_{1}<t<t_{2}$, and also a function of $\omega$, although such features are kept implicit, to simplify the notations. In what follows, we shall often use the more compact notations

$$
\mathcal{S}_{t_{2}, t_{1}}(\boldsymbol{x}, \boldsymbol{y}) \equiv\left\langle\hat{S}_{t_{2}, t_{1}}(\boldsymbol{x}, \boldsymbol{y})\right\rangle, \quad \mathcal{S}(\boldsymbol{x}, \boldsymbol{y}) \equiv \mathcal{S}_{\infty,-\infty}(\boldsymbol{x}, \boldsymbol{y})
$$

Before we proceed, let us open here a parenthesis on the generalization of the present results to finite $N_{c}$ : within the Gaussian approximation at hand, it is in fact possible to also evaluate the finite- $N_{c}$ corrections. (See e.g. refs. [37, 39-41, 43, 44] for corresponding discussions in the framework of the CGC.) To keep the presentation as simple as possible, we shall stick to the large- $N_{c}$ limit in all the intermediate steps, but indicate the generalization of the final results to finite $N_{c}$.

We now close the parenthesis and return to the evaluation of eq. (4.13) in the Gaussian approximation and at large $N_{c}$. The first term in the r.h.s. has been already discussed in eqs. (4.15)-(4.17). The remaining three terms can be similarly manipulated. Under the present assumptions, they all involve the same product of Wilson line correlators, as written down in the last line of eq. (4.15). Thus, they differ from each other (and from the first term) only via the actual values taken by the endpoints $\boldsymbol{r}_{1}$ and $\boldsymbol{r}_{2}$ of the emitted gluon. By putting together the previous results and adding the contribution of the 'virtual' piece (i.e. eq. (4.11) times $\mathcal{S}(\boldsymbol{x}, \boldsymbol{y})$ ), one obtains

$$
\begin{aligned}
\left\langle\Delta H \hat{S}_{\boldsymbol{x} \boldsymbol{y}}\right\rangle= & -\frac{\alpha_{s} N_{c}}{2} \int_{\omega}^{\omega_{c}} \frac{\mathrm{d} p^{+}}{\left(p^{+}\right)^{3}} \int_{-\infty}^{\infty} \mathrm{d} t_{2} \int_{-\infty}^{t_{2}} \mathrm{~d} t_{1} \partial_{\boldsymbol{r}_{1}}^{i} \partial_{\boldsymbol{r}_{2}}^{i}\left\{\int[\mathcal{D} \boldsymbol{r}(t)] \mathrm{e}^{\mathrm{i} \frac{p^{+}}{2} \int_{t_{1}}^{t_{2}} \mathrm{~d} t \dot{\boldsymbol{r}}^{2}(t)}\right. \\
& \left.\times \mathcal{S}_{\infty, t_{2}}(\boldsymbol{x}, \boldsymbol{y})\left[\mathcal{S}_{t_{2}, t_{1}}(\boldsymbol{x}, \boldsymbol{r}) \mathcal{S}_{t_{2}, t_{1}}(\boldsymbol{r}, \boldsymbol{y})-\mathcal{S}_{t_{2}, t_{1}}(\boldsymbol{x}, \boldsymbol{y})\right] \mathcal{S}_{t_{1},-\infty}(\boldsymbol{x}, \boldsymbol{y})\right\}\left.\left.\right|_{\boldsymbol{r}_{2}=\boldsymbol{y}} ^{\boldsymbol{r}_{2}=\boldsymbol{x}}\right|_{\boldsymbol{r}_{1}=\boldsymbol{y}} ^{\boldsymbol{r}_{1}=\boldsymbol{x}} .
\end{aligned}
$$

This equation can be recognized as a generalization of the BK equation (3.31), to which it reduces in the limit where the target is a shockwave. Namely, for a target localized near $x^{+}=0$, the BK equation is obtained from eq. (4.19) by integrating over positive 
values for $t_{2}$ and negative values for $t_{1}$. (When both $t_{1}$ and $t_{2}$ have the same sign, one has $\mathcal{S}_{t_{2}, t_{1}}=1$ for a $\mathrm{SW}$ target and then the r.h.s. of eq. (4.19) simply vanishes.) In that case, $\mathcal{S}_{\infty, t_{2}}=\mathcal{S}_{t_{1},-\infty}=1$, whereas $\mathcal{S}_{t_{2}, t_{1}}(\boldsymbol{x}, \boldsymbol{y})=\mathcal{S}(\boldsymbol{x}, \boldsymbol{y})$ is independent of time. Moreover, the $S$-matrices for the two daughter dipoles, $\mathcal{S}(\boldsymbol{x}, \boldsymbol{r})$ and $\mathcal{S}(\boldsymbol{r}, \boldsymbol{y})$, do not depend upon the detailed trajectory $\boldsymbol{r}(t)$ of the soft gluon, but only upon its position $\boldsymbol{r}(0)$ at the interaction time $t=0$. Hence one can write (compare to eq. (B.9))

$$
\mathcal{S}(\boldsymbol{x},[\boldsymbol{r}(t)]) \simeq \mathcal{S}(\boldsymbol{x}, \boldsymbol{r}(0))=\int \mathrm{d}^{2} \boldsymbol{z} \delta^{(2)}(\boldsymbol{z}-\boldsymbol{r}(0)) \mathcal{S}(\boldsymbol{x}, \boldsymbol{z})
$$

After also using the factorization property (B.10) for the free path integral, one can perform the time integrations as in eqs. (3.7), (3.8) and (3.28), then recognize the logarithmic enhancement of the ensuing integral over $p^{+}$, and finally reconstruct the BK equation (3.31), as anticipated. Eq. (4.20) expresses the revival of the eikonal approximation: the transverse diffusion of the gluon fluctuation can be neglected while crossing a 'shockwave' target with very narrow longitudinal extent.

Returning to the case of an extended target, we notice that eq. (4.19) is complicated by the fact that interactions are delocalized in time and also by the transverse diffusion of the gluon fluctuation, as encoded in the path integral. Accordingly, the time integrations in eq. (4.19) cannot be performed in full generality, i.e. without further approximations. Yet, as we shall shortly see, eq. (4.19) is a convenient starting point for further studies: it allows for explicit calculations in limiting situations of interest and also for general physics conclusions. Before we proceed with more specific studies, it is convenient to recast this expression in a more suggestive form.

First, one can interpret eq. (4.19) as an equation for the evolution w.r.t. the longitudinal momentum ('energy') $p^{+} \equiv \omega$ of the emitted gluon. To that aim, we shall write

$$
\left\langle\Delta H \hat{S}_{\boldsymbol{x} \boldsymbol{y}}\right\rangle=\Delta \mathcal{S}(\boldsymbol{x}, \boldsymbol{y}) \equiv \int_{\omega}^{\omega_{c}} \mathrm{~d} \omega_{1} \frac{\partial \mathcal{S}(\boldsymbol{x}, \boldsymbol{y})}{\partial \omega_{1}},
$$

which by comparison with eq. (4.19) allows us to deduce the following evolution equation

$$
\begin{aligned}
-\frac{\partial \ln \mathcal{S}(\boldsymbol{x}, \boldsymbol{y})}{\partial \omega}=\frac{\alpha_{s} N_{c}}{2} & \frac{1}{\omega^{3}} \int_{-\infty}^{\infty} \mathrm{d} t_{2} \int_{-\infty}^{t_{2}} \mathrm{~d} t_{1} \partial_{\boldsymbol{r}_{1}}^{i} \partial_{\boldsymbol{r}_{2}}^{i}\left\{\int[\mathcal{D} \boldsymbol{r}(t)] \mathrm{e}^{\mathrm{i} \frac{\omega}{2} \int_{t_{1}}^{t_{2}} \mathrm{~d} t \dot{\boldsymbol{r}}^{2}(t)}\right. \\
\times & {\left.\left[\mathcal{S}_{t_{2}, t_{1}}(\boldsymbol{x}, \boldsymbol{r}) \mathcal{S}_{t_{2}, t_{1}}(\boldsymbol{r}, \boldsymbol{y}) \mathcal{S}_{t_{2}, t_{1}}^{-1}(\boldsymbol{x}, \boldsymbol{y})-1\right]\right\}\left.\left.\right|_{\boldsymbol{r}_{2}=\boldsymbol{y}} ^{\boldsymbol{y}_{2}=\boldsymbol{x}}\right|_{\boldsymbol{r}_{1}=\boldsymbol{y}} ^{\boldsymbol{r}_{1}=\boldsymbol{x}} }
\end{aligned}
$$

(Recall that $0<\mathcal{S} \leq 1$, so $\ln \mathcal{S}<0$.) eq. (4.22) is somewhat formal because, so far, we have not demonstrated the logarithmic enhancement $\int(\mathrm{d} \omega / \omega)$ of the soft gluon emission for the case of an extended target. Yet, as we shall see starting with section 4.3, such enhancement shows up indeed in all the cases where we will be able to perform the time integrations.

Furthermore, we anticipate that the dominant corrections in the regime of interest are associated with very soft gluons, with energies $p^{+} \ll \omega_{c}$. Such gluons have small 
lifetimes $\tau_{c o h} \ll L$, hence they are typically emitted and absorbed deeply inside the medium: boundary effects, i.e. emissions which occur within a distance $\sim \tau_{c o h}$ from the medium edges at $x^{+}=0$ or $x^{+}=L$, are comparatively suppressed by a factor $\tau_{c o h} / L \ll 1$. Accordingly, it is justified to restrict the time integrations in eq. (4.22) to $0<t_{1}<t_{2}<L$. In this range, one can exploit the Gaussian nature of the medium correlations, cf. eq. (4.14), to express the dipole $S$-matrix as (compare to eq. (4.5))

$$
\mathcal{S}_{t_{2}, t_{1}}(\boldsymbol{x}, \boldsymbol{y} ; \omega)=\exp \left\{-g^{2} C_{F} \int_{t_{1}}^{t_{2}} \mathrm{~d} t \Gamma_{\omega}(t, \boldsymbol{x}-\boldsymbol{y})\right\}=\exp \left\{-g^{2} C_{F}\left(t_{2}-t_{1}\right) \Gamma_{\omega}(\boldsymbol{x}-\boldsymbol{y})\right\}
$$

where $\Gamma_{\omega}(t, \boldsymbol{x}-\boldsymbol{y}) \equiv \bar{\Gamma}_{\omega}(t, \mathbf{0})-\bar{\Gamma}_{\omega}(t, \boldsymbol{x}-\boldsymbol{y})$. The second equality in eq. (4.23) holds for a medium which is homogeneous in time, a case to which we shall restrict ourselves in what follows. In particular, $\mathcal{S}(\boldsymbol{x}, \boldsymbol{y})$ is obtained by replacing $t_{2}-t_{1} \rightarrow L$ in eq. (4.23). Note that the quantity $g^{2} C_{F} \Gamma_{\omega}(\boldsymbol{x}-\boldsymbol{y})$ is the dipole amplitude for one scattering per unit length (or time).

After using eq. (4.23) and restricting the time integrations to the support of the target, eq. (4.22) can be rewritten as an equation for $\Gamma_{\omega}(\boldsymbol{x}-\boldsymbol{y})$ :

$$
\begin{aligned}
L & \frac{\partial \Gamma_{\omega}(\boldsymbol{x}, \boldsymbol{y})}{\partial \omega} \\
= & \frac{1}{4 \pi \omega^{3}} \int_{0}^{L} \mathrm{~d} t_{2} \int_{0}^{t_{2}} \mathrm{~d} t_{1} \partial_{\boldsymbol{r}_{1}}^{i} \partial_{\boldsymbol{r}_{2}}^{i}\left\{\int[\mathcal{D} \boldsymbol{r}] \mathrm{e}^{\mathrm{i} \frac{\omega}{2} \int_{t_{1}}^{t_{2}} \mathrm{~d} t^{\prime} \dot{\boldsymbol{r}}^{2}\left(t^{\prime}\right)}\right. \\
& \left.\times\left[\exp \left(-\frac{g^{2} N_{c}}{2} \int_{t_{1}}^{t_{2}} \mathrm{~d} t\left[\Gamma_{\omega}(\boldsymbol{x}, \boldsymbol{r}(t))+\Gamma_{\omega}(\boldsymbol{r}(t), \boldsymbol{y})-\Gamma_{\omega}(\boldsymbol{x}, \boldsymbol{y})\right]\right)-1\right]\right\}\left.\left.\right|_{\boldsymbol{r}_{2}=\boldsymbol{y}} ^{\boldsymbol{r}_{2}=\boldsymbol{x}}\right|_{\boldsymbol{r}_{1}=\boldsymbol{y}} ^{\boldsymbol{r}_{1}=\boldsymbol{x}} .
\end{aligned}
$$

We have also used $C_{F} \simeq N_{c} / 2$, as appropriate at large $N_{c}$.

It is in fact easy to generalize the above results to finite $N_{c}$ and also to a generic representation $R$ for the original color dipole (see appendix D for details): within the limits of the Gaussian approximation (4.14), the average $S$-matrix for an $R \bar{R}$-dipole and for finite $N_{c}$ is given by eq. (4.23) with $C_{F} \rightarrow C_{R}$ (the second Casimir for the respective representation) and with the function $\Gamma_{\omega}(\boldsymbol{x}-\boldsymbol{y})$ obeying exactly the same equation as above, i.e. eq. (4.24). Such a simplification has been previously noticed in ref. [40], where the analog of eq. (4.24) has been proposed in the context of a shockwave target (that is, as a mean field approximation to JIMWLK evolution at finite $N_{c}$ ).

Note finally that eq. (4.24) can be viewed as a generalization (and also a corrected version) of a corresponding result in ref. [9], as shown in eqs. (11-12) there. ${ }^{11}$ eq. (4.24) is more general because it is an evolution equation, whose solution (say, as obtained via

\footnotetext{
${ }^{11}$ For the sake of this comparaison, note that the quantities denoted as $S\left(x_{\perp}\right)$ and $N\left(x_{\perp}, \omega\right)$ in [9] correspond to our present quantities $\Delta \mathcal{S}(\boldsymbol{x}, \boldsymbol{y})$ and respectively $\omega(\partial \mathcal{S} / \partial \omega)$, cf. eq. (4.21). Hence, eq. (12) in [9] must be compared to the equation obtained by multiplying both sides of our eq. (4.24) by a factor $\left[-\omega\left(g^{2} N_{c} / 2\right) \mathcal{S}(\boldsymbol{x}, \boldsymbol{y})\right]$.
} 
successive iterations) would resum an infinite series of radiative corrections of arbitrary loop order (within the high-energy approximations at hand). By comparison, eq. (12) in [9] is a one-loop result, which can be viewed as the first iteration of our eq. (4.24) - the limit in which the r.h.s. of the latter is evaluated in the tree-level approximation (i.e., by using the expression (4.5) for the average $S$-matrix). Moreover, even at one-loop order, eq. (12) in [9] mistreats the 'virtual' corrections: instead of subtracting a term proportional to $\mathcal{S}(\boldsymbol{x}, \boldsymbol{y})$, as required by the correct prescription in eq. (2.18), the authors of ref. [9] have merely subtracted the vacuum limit $\left(A^{-} \rightarrow 0\right)$ of the corresponding 'real' term. ${ }^{12}$ This imprecision has consequences to leading logarithmic accuracy, as we shall see in the next subsection. (In ref. [9], the proper virtual term has been heuristically added in the calculation of the single-logarithmic corrections, where it was actually needed.)

\subsection{The single scattering approximation}

In this section, we shall discuss a series of successive approximations to eq. (4.24), which will lead us to a simpler, linear, equation - namely, eq. (4.43) — which, besides being more tractable (including via analytic methods), has also the virtue to capture the dominant radiative corrections in the limit where the medium is large - those which are enhanced by the double logarithm $\ln ^{2}(L / \lambda)$, with $\lambda$ a microscopic length scale to be explained in section 4.3.3.

To better appreciate the ensuing approximations, let us remind here that we are primarily interested in the radiative corrections which are enhanced by (at least) one large energy logarithm $\ln \left(\omega_{c} / \omega\right)$, or, equivalently, which exhibit a logarithmic divergence in the soft limit $\omega \rightarrow 0$. Since the lifetime $\tau_{c o h}$ of the fluctuations is proportional to $\omega$ and, moreover, their scattering amplitude in the medium is proportional to $\tau_{c o h}$, we conclude that fluctuations which are sufficiently soft should scatter only weakly. This explains our current emphasis on the single scattering approximation. Yet, the situation turns out to be more subtle: both the lifetime $\tau_{c o h}=2 \omega / p_{\perp}^{2}$ and the scattering amplitude per unit time also depend upon the transverse momentum $p_{\perp}$ (or the transverse size $B_{\perp} \sim 1 / p_{\perp}$ ) of the fluctuation. Hence relative hard (large $\omega$ ) fluctuations can still interact only weakly provided their size is small enough. This implies the existence of double-logarithmic corrections associated with single scattering, where the second logarithm comes from integrating over the transverse phase-space [9]. Such corrections will be explicitly identified and resumed in our formalism, with results which agree with ref. [9]. Vice-versa, single energy logarithms can be generated also via multiple scattering, namely, via fluctuations with generic energies $\omega \ll \omega_{c}$ and with sufficiently large transverse sizes. Such corrections too are encoded in our formalism, but it seems very difficult to explicitly isolate them (beyond the one-loop approximation already considered in ref. [9]), for reasons to be explained in section 4.5.

\subsubsection{The BFKL equation for jet quenching}

Let us first recall the interesting kinematical regime for a study of the transverse momentum broadening and of its high-energy evolution: the transverse size $r \equiv|\boldsymbol{x}-\boldsymbol{y}|$ of the original

\footnotetext{
${ }^{12}$ In our present set-up, the procedure of ref. [9] would amount to subtracting just the coefficient (4.11) of the virtual term, and not the product between that coefficient and the average $S$-matrix $\mathcal{S}(\boldsymbol{x}, \boldsymbol{y})$ of the unevolved dipole.
} 
dipole is parametrically of order $1 / Q_{s}$, with $Q_{s}^{2}=\hat{q} L$, and the typical energies of the emitted gluons obey $\omega \ll \omega_{c}$, with $\omega_{c}=\hat{q} L^{2}$. This particular regime corresponds to the unitarity line for the dipole amplitude, or, equivalently, to the plasma saturation line (see the discussion in section 4.4).

Under these assumptions, we shall focus on the regime where the quark-gluon ('twodipole') fluctuation living during the time interval $\Delta t=t_{2}-t_{1}$ undergoes a single collision with the medium, as illustrated in figure 2 . We shall refer to this regime as the 'single scattering approximation', but one should keep in mind that multiple scattering is still allowed prior to, and after, the fluctuation, that is, during the comparatively large time intervals between 0 and $t_{1}$ and, respectively, between $t_{2}$ and $L$. Roughly speaking, this approximation is justified provided the transverse separation between the soft gluon and the parent dipole is small enough (see eq. (4.32) below). Within this 'single scattering' regime, one can expand the exponential within the square brackets in eq. (4.24) to linear order:

$$
\begin{aligned}
L \frac{\partial \Gamma_{\omega}(\boldsymbol{x}, \boldsymbol{y})}{\partial \omega}=-\frac{\alpha_{s} N_{c}}{2} & \frac{1}{\omega^{3}} \int_{0}^{L} \mathrm{~d} t_{2} \int_{0}^{t_{2}} \mathrm{~d} t_{1} \partial_{\boldsymbol{r}_{1}}^{i} \partial_{\boldsymbol{r}_{2}}^{i}\left\{\int[\mathcal{D} \boldsymbol{r}] \mathrm{e}^{\mathrm{i} \frac{\omega}{2} \int_{t_{1}}^{t_{2}} \mathrm{~d} t^{\prime} \dot{\boldsymbol{r}}^{2}\left(t^{\prime}\right)}\right. \\
& \left.\times \int_{t_{1}}^{t_{2}} \mathrm{~d} t\left[\Gamma_{\omega}(\boldsymbol{x}, \boldsymbol{r}(t))+\Gamma_{\omega}(\boldsymbol{r}(t), \boldsymbol{y})-\Gamma_{\omega}(\boldsymbol{x}, \boldsymbol{y})\right]\right\}\left.\left.\right|_{\boldsymbol{r}_{2}=\boldsymbol{y}} ^{\boldsymbol{r}_{2}=\boldsymbol{x}}\right|_{\boldsymbol{r}_{1}=\boldsymbol{y}} ^{\boldsymbol{r}_{1}=\boldsymbol{x}}
\end{aligned}
$$

For the physical interpretation of this equation, one should keep in mind that the quantity $g^{2} C_{F} \Gamma_{\omega}$ is the single scattering amplitude per unit time (or length). Hence, the solution to eq. (4.25) shows how each of the scatterings suffered by the original dipole $(\boldsymbol{x}, \boldsymbol{y})$ is renormalized by the high-energy evolution. By using this solution within eq. (4.23), one obtains the $S$-matrix for that dipole in the presence of both multiple scattering and highenergy evolution.

A main virtue of eq. (4.25) is that the time integrals over $t_{1}, t_{2}$, and $t$ can be explicitly performed, as we now explain. An important consequence of this calculation will be to render manifest the logarithmic enhancement of the radiative corrections with small $\omega$. To that aim, it is convenient to reverse the order of the time integrations, as follows:

$$
\int_{0}^{L} \mathrm{~d} t_{2} \int_{0}^{t_{2}} \mathrm{~d} t_{1} \int_{t_{1}}^{t_{2}} \mathrm{~d} t=\int_{0}^{L} \mathrm{~d} t \int_{0}^{t} \mathrm{~d} t_{1} \int_{t}^{L} \mathrm{~d} t_{2}
$$

After introducing the identity in the form $1=\int \mathrm{d}^{2} \boldsymbol{z} \delta^{(2)}(\boldsymbol{z}-\boldsymbol{r}(t))$ and using eq. (B.10) with $\boldsymbol{r}(0) \rightarrow \boldsymbol{r}(t)$, eq. (4.25) becomes

$$
\begin{aligned}
L & \frac{\partial \Gamma_{\omega}(\boldsymbol{x}, \boldsymbol{y})}{\partial \omega} \\
= & -\frac{\alpha_{s} N_{c}}{2} \frac{1}{\omega^{3}} \int \mathrm{d}^{2} \boldsymbol{z}\left[\Gamma_{\omega}(\boldsymbol{x}, \boldsymbol{z})+\Gamma_{\omega}(\boldsymbol{z}, \boldsymbol{y})-\Gamma_{\omega}(\boldsymbol{x}, \boldsymbol{y})\right] \\
& \times\left.\left.\int_{0}^{L} \mathrm{~d} t \int_{0}^{t} \mathrm{~d} t_{1} \int_{t}^{L} \mathrm{~d} t_{2} \partial_{\boldsymbol{r}_{1}}^{i} \partial_{\boldsymbol{r}_{2}}^{i}\left\{\mathcal{G}_{0}\left(t_{2}-t, \boldsymbol{r}_{2}-\boldsymbol{z} ; \omega\right) \mathcal{G}_{0}\left(t-t_{1}, \boldsymbol{z}-\boldsymbol{r}_{1} ; \omega\right)\right\}\right|_{\boldsymbol{r}_{2}=\boldsymbol{y}} ^{\boldsymbol{r}_{2}=\boldsymbol{x}}\right|_{\boldsymbol{r}_{1}=\boldsymbol{y}} ^{\boldsymbol{r}_{1}=\boldsymbol{x}} .
\end{aligned}
$$


The time integrations can now be performed as in eqs. (3.7)-(3.8). For instance,

$$
\begin{aligned}
\frac{1}{2 \omega} \int_{t}^{L} \mathrm{~d} t_{2} \partial_{\boldsymbol{r}_{2}}^{i} \mathcal{G}_{0}\left(t_{2}-t, \boldsymbol{r}_{2}-\boldsymbol{z} ; \omega\right) & =\frac{-\mathrm{i}}{2 \omega} \int \frac{\mathrm{d}^{2} \boldsymbol{p}}{(2 \pi)^{2}} p^{i} \mathrm{e}^{\mathrm{i} \boldsymbol{p} \cdot\left(\boldsymbol{r}_{2}-\boldsymbol{z}\right)} \int_{t}^{L} \mathrm{~d} t_{2} \mathrm{e}^{-\mathrm{i} \frac{p_{\perp}^{2}}{2 \omega}\left(t_{2}-t\right)} \\
& =-\int \frac{\mathrm{d}^{2} \boldsymbol{p}}{(2 \pi)^{2}} \frac{p^{i}}{p_{\perp}^{2}} \mathrm{e}^{\mathrm{i} \boldsymbol{p} \cdot\left(\boldsymbol{r}_{2}-\boldsymbol{z}\right)}\left[1-\mathrm{e}^{-\mathrm{i} \frac{p_{\perp}^{2}}{2 \omega}(L-t)}\right] \\
& \simeq-\int \frac{\mathrm{d}^{2} \boldsymbol{p}}{(2 \pi)^{2}} \frac{p^{i}}{p_{\perp}^{2}} \mathrm{e}^{\mathrm{i} \boldsymbol{p} \cdot\left(\boldsymbol{r}_{2}-\boldsymbol{z}\right)}=\frac{\mathrm{i}}{2 \pi} \frac{r_{2}^{i}-z^{i}}{\left(\boldsymbol{r}_{2}-\boldsymbol{z}\right)^{2}} .
\end{aligned}
$$

The crucial approximation that has been performed here, was to neglect the rapidlyoscillating complex exponential in the second line. This is indeed justified for the problem at hand because $L-t \sim L$ is much larger than the lifetime $\tau_{c o h}=2 \omega / p_{\perp}^{2}$ of the interesting gluon fluctuations. Accordingly, the final result in eq. (4.28) is independent of $t$. This final result is recognized as the Weizsäcker-Williams (or BFKL) wavefunction of the valence quark at $\boldsymbol{r}_{2}$ (with $\boldsymbol{r}_{2}=\boldsymbol{x}$, or $\boldsymbol{r}_{2}=\boldsymbol{y}$ ) accompanied by a soft gluon at $\boldsymbol{z}$. At this level, the transverse diffusion (which was a priori encoded in the gluon propagator) is not manifest anymore: this has been averaged out by the integral over the emission time $t_{2}$, because the latter had a sufficiently large phase-space at its disposal: $L-t \gg \tau_{c o h}$. This argument explains why the transverse diffusion plays no role in this single scattering approximation: the interaction probability being weak, the (unique) scattering typically occurs far away from the gluon emission vertices, at a time where the soft gluon wavefunction is already fully developed.

A similar reasoning applies to the integral over $t_{1}$, whose result can be read off eq. (3.8). The final integral over $t$ then simply yields a factor of $L$, which cancels the similar factor in the 1.h.s. of eq. (4.25). The above discussion is quite similar to that of the 'crossing' piece in the evolution when the target is a shockwave, cf. section 3.1. As in that case, the two integrals over the emission times $t_{1}$ and $t_{2}$ around the interaction time $t$ have generated a factor $\tau_{c o h}^{2} \propto \omega^{2}$, which, when also combined with the overall factor $1 / \omega^{3}$ in the r.h.s. of eq. (4.25), produces a final result $\propto 1 / \omega$. This demonstrates the logarithmic enhancement, as anticipated. We are thus lead to the following, relatively simple, equation for $\Gamma_{\omega}$ (with $\bar{\alpha}=\alpha_{s} N_{c} / \pi$ and the kernel $\mathcal{M}_{\boldsymbol{x} \boldsymbol{y} \boldsymbol{z}}$ as defined in eq. (3.24)):

$$
\omega \frac{\partial \Gamma_{\omega}(\boldsymbol{x}, \boldsymbol{y})}{\partial \omega}=\frac{\bar{\alpha}}{2 \pi} \int_{\boldsymbol{z}} \mathcal{M}_{\boldsymbol{x} \boldsymbol{y} \boldsymbol{z}}\left[\Gamma_{\omega}(\boldsymbol{x}, \boldsymbol{z})+\Gamma_{\omega}(\boldsymbol{z}, \boldsymbol{y})-\Gamma_{\omega}(\boldsymbol{x}, \boldsymbol{y})\right]
$$

This equation looks formally similar to the BFKL equation eq. (3.30), but in reality it is different from the latter, in that it has a different validity range (as characterized by the boundary conditions for the integral over $\boldsymbol{z}$; see below) and it applies in different circumstances.

Namely, recall that eq. (3.30) applies to a dipole scattering off a shockwave and is valid so long as the amplitude for a single scattering remains small: $\mathcal{T}_{Y} \ll 1$. This condition refers to both the parent dipole, with size $|\boldsymbol{x}-\boldsymbol{y}|$, and the daughter dipoles, $|\boldsymbol{x}-\boldsymbol{z}|$ and $|\boldsymbol{z}-\boldsymbol{y}|$, and it implies that all these dipoles must be small relative to the same scale the target saturation momentum $Q_{s}(Y)$. Moreover this condition $r \ll 1 / Q_{s}(Y)$ is only weakly dependent upon the energy $\omega$ of the fluctuation (or upon $Y$ ), in the sense that 


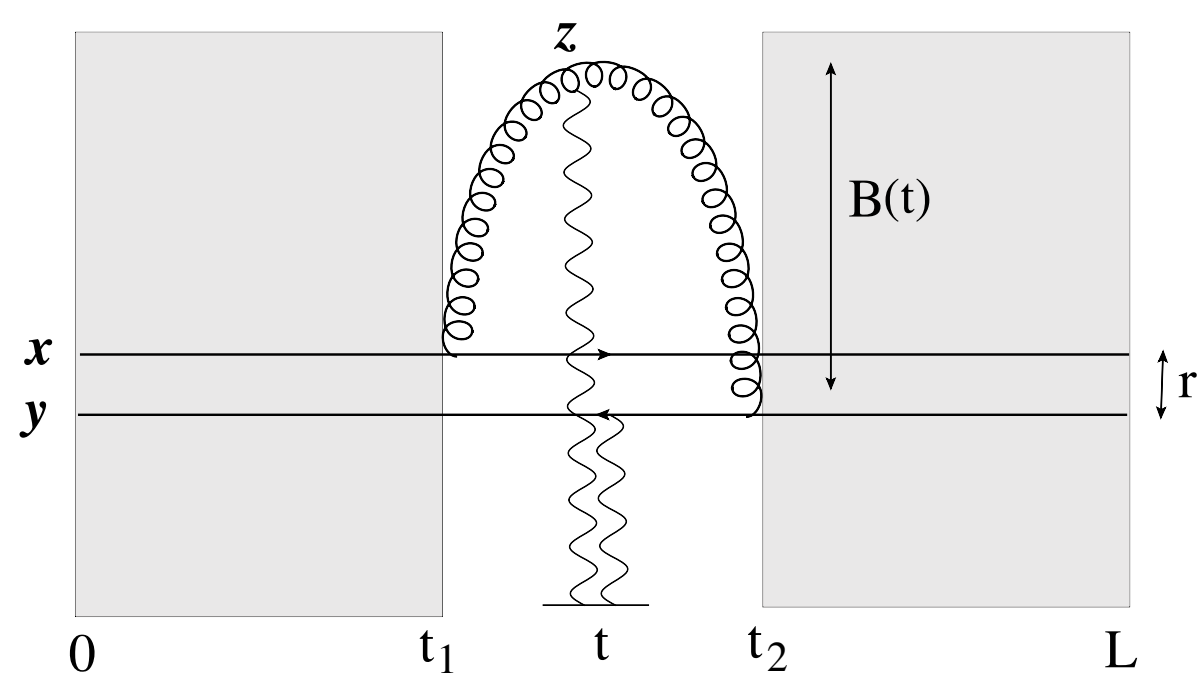

Figure 2. A diagram for dipole evolution in the single scattering approximation, cf. eqs. (4.27) and (4.29). The grey areas prior and after the fluctuation are regions of multiple scattering. During the fluctuation, the 3-parton $(q \bar{q} g)$ system scatters only once, at some intermediate time $t$. The lifetime $\Delta t=t_{2}-t_{1}$ of the fluctuation is considerably smaller than $L$; its transverse size $B_{\perp}$ is typically much larger than the size $r$ of the original dipole, but much smaller than the 'saturation' size $2 / k_{\mathrm{br}}(\omega)$ introduced by multiple scattering.

this dependence is introduced by the high energy evolution and hence it vanishes in the weak-coupling limit $\bar{\alpha} \rightarrow 0$.

By contrast, eq. (4.29) refers to the scattering amplitude per unit time and is valid so long as the scattering accumulated by the fluctuation (i.e. by the daughter dipoles) during its lifetime $\Delta t$ remains small. Since $\Delta t$ is much smaller than the size $L$ of the medium, this condition allows the daughter dipoles to be much larger than the parent one. Moreover, the upper limit thus introduced on the transverse size of the fluctuation is strongly dependent upon the energy, since so is its lifetime $\Delta t \propto \omega$. Specifically, the weak-scattering condition for the fluctuation can be written as

$$
2 g^{2} C_{F} \Delta t \Gamma_{\omega}\left(B_{\perp}\right) \ll 1
$$

where $B_{\perp}$ is the maximal size of any of the daughter dipoles during $\Delta t-$ that is, the largest among the distances $|\boldsymbol{x}-\boldsymbol{r}(t)|$ and $|\boldsymbol{r}(t)-\boldsymbol{y}|$ for $t_{1}<t<t_{2}$ - and the overall factor of 2 stands for the two daughter dipoles (see also figure 2). As we shall shortly check, this $B_{\perp}$ is typically much larger than the size $r \sim 1 / Q_{s}$ of the original dipole, hence it is indeed justified to indistinguishably treat the two daughter dipoles.

To be more specific, let us estimate the fluctuation lifetime via the uncertainty principle, $\Delta t \simeq 2 \omega / p_{\perp}^{2} \simeq \omega B_{\perp}^{2} / 2$, and use the tree-level estimate for $\Gamma$ in eq. (4.5): $g^{2} C_{F} \Gamma\left(B_{\perp}\right) \simeq \hat{q}\left(1 / B_{\perp}^{2}\right) B_{\perp}^{2} / 4$. Then the condition (4.30) can be rewritten as an energydependent upper limit on $B_{\perp}^{2}$ :

$$
B_{\perp}^{2} \ll \frac{4}{2 \sqrt{\omega \hat{q}}} \equiv \frac{4}{k_{\mathrm{br}}^{2}(\omega)}
$$


with $\hat{q}$ itself evaluated on the scale set by this limit: $\hat{q}=\hat{q}\left(k_{\mathrm{br}}^{2}\right)$. This constraint will be confirmed by the analysis in section 4.5 , which also shows that the very large fluctuations, with sizes $B_{\perp} \gtrsim 2 / k_{\mathrm{br}}(\omega)$, 一 or, equivalently, with large lifetimes $\Delta t \gtrsim \sqrt{\omega / \hat{q}}-$ are destroyed by multiple scattering. On the other hand, the very small fluctuations, with transverse sizes much smaller than $r \sim 2 / Q_{s}$, do not contribute to the evolution, since their effects cancel between the 'real' and 'virtual' terms in eq. (4.29). To conclude, the phase-space for the single scattering approximation reads

$$
r \simeq \frac{2}{Q_{s}} \lesssim|\boldsymbol{x}-\boldsymbol{z}|,|\boldsymbol{z}-\boldsymbol{y}| \ll \frac{2}{k_{\mathrm{br}}(\omega)} .
$$

Via the uncertainty principle, eq. (4.32) implies that the transverse momentum $p_{\perp}$ of the emitted gluons lies within the range $k_{\mathrm{br}}(\omega) \ll p_{\perp} \lesssim Q_{s}$. This phase-space depends upon the energy $\omega$ of the emitted gluons, so it is important to recall that the interesting fluctuations have $\omega \ll \omega_{c}$. When $\omega$ approaches the upper limit $\omega_{c}$, the phase-space in eq. (4.32) shrinks to zero since $k_{\mathrm{br}}\left(\omega_{c}\right) \sim Q_{s}$. Vice-versa, for the soft gluons with $\omega \ll \omega_{c}$, one has $k_{\mathrm{br}}^{2}(\omega) \ll Q_{s}^{2}$ and then the transverse phase-space for linear evolution is indeed very large.

To summarize, the main difference w.r.t. the 'standard' BFKL equation is that, in the context of eq. (4.29), the longitudinal and transverse phase-spaces are strongly correlated with each other, already in the absence of the evolution (i.e. in the weak-coupling limit $\bar{\alpha} \rightarrow$ $0)$. This has important consequences for the evolution, to be discussed in the next section.

\subsubsection{The double logarithmic approximation}

The previous arguments show that, when increasing the longitudinal phase-space for the evolution by decreasing $\omega$ below $\omega_{c}$, one simultaneously increases the corresponding transverse phase-space, by decreasing the lower limit $k_{\mathrm{br}}(\omega)$ on the transverse momentum $p_{\perp}$ of the fluctuations (or, equivalently, increasing the upper limit on their transverse size, cf. eq. (4.32)). In view of this and of the well-known fact that the BFKL evolution admits a double-logarithmic regime [18], it is clear that the radiative corrections can be enhanced not just by the large energy logarithm $\ln \left(\omega_{c} / \omega\right)$, but also by the even larger double logarithm $\ln \left(\omega_{c} / \omega\right) \ln \left(Q_{s}^{2} / k_{\mathrm{br}}^{2}(\omega)=(1 / 2) \ln ^{2}\left(\omega_{c} / \omega\right)\right.$. This enhancement has been previously recognized in ref. [9], where the respective correction to the transverse momentum broadening has been first computed (see also [14, 71]).

In what follows, we shall derive a simplified version of eq. (4.29), which resums the dominant, double-logarithmic, corrections alone. To that aim, we need to focus on the relatively large fluctuations with $|\boldsymbol{x}-\boldsymbol{z}| \simeq|\boldsymbol{z}-\boldsymbol{y}| \gg r$. Since the dipole scattering amplitude $\Gamma_{\omega}(\boldsymbol{x}, \boldsymbol{z})$ is a rapidly growing function of the dipole size $|\boldsymbol{x}-\boldsymbol{z}|$ (see below), it is quite clear that, in this regime, one can neglect the 'virtual' term $\propto \Gamma_{\omega}(\boldsymbol{x}, \boldsymbol{y})$ in eq. (4.29). Then this equation simplifies to

$$
\omega \frac{\partial \Gamma_{\omega}(r)}{\partial \omega} \simeq \bar{\alpha} \int \mathrm{d} B_{\perp}^{2} \frac{r^{2}}{B_{\perp}^{4}} \Gamma_{\omega}\left(B_{\perp}\right)
$$

where we have used $B_{\perp}$ to denote the size of any of the two daughter dipoles. The initial condition for this equation at $\omega \simeq \omega_{c}$, i.e. the tree-level result in eq. (4.5), is roughly 
proportional to the dipole size squared. Remarkably, eq. (4.33) shows that this property is preserved by the evolution under the approximations of interest. Hence, we can write

$$
g^{2} C_{F} \Gamma_{\omega}\left(B_{\perp}\right) \equiv \frac{1}{4} \hat{q}_{\omega}\left(1 / B_{\perp}^{2}\right) B_{\perp}^{2},
$$

where the function $\hat{q}_{\omega}\left(1 / B_{\perp}^{2}\right)$ has only a weak dependence upon $B_{\perp}^{2}$ (for $\omega \simeq \omega_{c}$, it reduces to the zeroth order result in eq. (4.6)). Then eq. (4.33) implies the following equation for $\hat{q}_{\omega}$,

$$
\omega \frac{\partial \hat{q}_{\omega}\left(1 / r^{2}\right)}{\partial \omega} \simeq \bar{\alpha} \int_{r^{2}}^{4 / k_{\mathrm{br}}^{2}(\omega)} \frac{\mathrm{d} B_{\perp}^{2}}{B_{\perp}^{2}} \hat{q}_{\omega}\left(1 / B_{\perp}^{2}\right)
$$

where the limits in the integral over $B_{\perp}^{2}$ follow from the previous discussion. Eq. (4.35) looks similar to the familiar 'double-logarithmic approximation' (DLA) to the BFKL equation (see e.g. [18]). But one should keep in mind that the upper limit in the above integral, which is energy-dependent, is specific to the problem at hand and reflects the non-linear physics of multiple scattering. (The standard DLA equation would be obtained by replacing this limit by some fixed, infrared, cutoff, like the confinement scale: $4 / k_{\mathrm{br}}^{2}(\omega) \rightarrow 1 / \Lambda^{2}$.) For this particular problem, and unlike in more standard applications of the BFKL equation to highenergy scattering $[18,31-33]$, the DLA encompasses the dominant radiative corrections in the high-energy limit $\omega_{c} / \omega \gg 1$, for any $r \lesssim 1 / Q_{s}$.

Importantly, the approximation (4.34) for the dipole amplitude does not introduce a (non-perturbative) anomalous dimension: the dominant behavior at small $r$ is still $r^{2}$, like at tree-level. The would-be 'collinear' divergence at large $B_{\perp}$ is cut off by multiple scattering. In turn, this implies that the radiative corrections that we are about to compute are mild enough to be absorbed into a redefinition of $\hat{q}$. Indeed, with this approximation for $\Gamma_{\omega}$, the evolved dipole $S$-matrix in eq. (4.23) has the same formal structure as at tree-level, namely (compare to eq. (4.5))

$$
\mathcal{S}_{\omega}(\boldsymbol{r}) \simeq \exp \left\{-\frac{1}{4} L \hat{q}_{\omega}\left(1 / r^{2}\right) \boldsymbol{r}^{2}\right\}
$$

This is in particular true for the values $r \sim 1 / Q_{s}$ which control the Fourier transform in eq. (4.1). In turn, this implies that the quark spectrum has the Gaussian form in eq. (4.8), but with a renormalized, energy-dependent, saturation momentum, defined by $Q_{s}^{2}=\hat{q}_{\omega}\left(Q_{s}^{2}\right) L$.

Consider now the first iteration to eq. (4.35) and assume for simplicity that the respective zeroth order result (to be denoted as $\hat{q}^{(0)}$ in what follows) is scale-independent. The first order correction implied by eq. (4.35) reads ${ }^{13}$

$$
\begin{aligned}
\delta \hat{q}_{\omega}^{(1)}\left(Q_{s}^{2}\right) & =\bar{\alpha} \hat{q}^{(0)} \int_{\omega}^{\omega_{c}} \frac{\mathrm{d} \omega_{1}}{\omega_{1}} \int_{k_{\mathrm{br}}^{2}\left(\omega_{1}\right)}^{Q_{s}^{2}} \frac{\mathrm{d} p_{\perp}^{2}}{p_{\perp}^{2}}=\bar{\alpha} \hat{q}^{(0)} \int_{\omega}^{\omega_{c}} \frac{\mathrm{d} \omega_{1}}{\omega_{1}} \ln \frac{Q_{s}^{2}}{k_{\mathrm{br}}^{2}\left(\omega_{1}\right)} \\
& \simeq \frac{\bar{\alpha}}{2} \hat{q}^{(0)} \int_{\omega}^{\omega_{c}} \frac{\mathrm{d} \omega_{1}}{\omega_{1}} \ln \frac{\omega_{c}}{\omega_{1}}=\frac{\bar{\alpha}}{4} \hat{q}^{(0)} \ln ^{2} \frac{\omega_{c}}{\omega},
\end{aligned}
$$

\footnotetext{
${ }^{13}$ To the accuracy of interest, one can replace $\hat{q} \simeq \hat{q}^{(0)}$ within the argument of the logarithm.
} 
where the approximate equality sign refers to the double-logarithmic accuracy and we preferred to use the transverse momentum variable $p_{\perp}^{2} \equiv 4 / B_{\perp}^{2}$ as an integration variable, instead of $B_{\perp}^{2}$. As expected, this correction is of order $\bar{\alpha}$, but it is enhanced by the possibly large double logarithm $\ln ^{2}\left(\omega_{c} / \omega\right)$. To understand how large can this actually be, one needs to know what is the minimal value for $\omega$ allowed on physical grounds. This issue has been previously addressed in ref. [9], where one has argued that this minimal value is controlled by a lower limit $\lambda$ on the lifetime $\tau_{c o h}=2 \omega / p_{\perp}^{2}$ of the fluctuations, which is independent of $L$. In the next subsection, we shall revisit and complete the arguments in ref. [9] and thus clarify the physical origin and the value of $\lambda$. But for the time being, it suffices to know that such a cutoff exists and examine its consequences. As we now explain, this implies the existence of a large phase-space for double-logarithmic evolution in the regime where $L \gg \lambda$.

Specifically, the condition $\tau_{c o h}>\lambda$ implies a lower limit on the gluon energy, $\omega>$ $\lambda p_{\perp}^{2} / 2$, which also depends upon its transverse momentum $p_{\perp}$. This last feature forces us to modify our previous analysis leading to eq. (4.37). Indeed, in the integral over $p_{\perp}^{2}$ within that equation, we have assumed that the maximal limit is equal to $Q_{s}^{2}$, but in reality this cannot exceed a value $p_{\perp \max }^{2}=2 \omega_{1} / \lambda$ which also depends upon $\omega_{1}$ (the other integration variable there). That is, the proper integration range in $p_{\perp}^{2}$ for a given value of $\omega$ is

$$
k_{\mathrm{br}}^{2}(\omega) \ll p_{\perp}^{2} \ll \min \left(\frac{2 \omega}{\lambda}, Q_{s}^{2}\right) .
$$

The upper limit introduces two constraints. First, it implies the necessary condition $2 \omega / \lambda \gg k_{\mathrm{br}}^{2}(\omega)$, or equivalently $\omega \gg \omega_{0} \equiv \hat{q} \lambda^{2}$, showing that $\omega_{0}$ is the absolute lower limit on the energy $\omega$ of the fluctuation. Second, it means that, when performing the integrals over the phase-space, one must distinguish between two different ranges in $\omega$, namely $\omega_{0}<\omega<\omega_{*}$ and $\omega_{*}<\omega<\omega_{c}$, where $\omega_{*} \equiv Q_{s}^{2} \lambda / 2$ is the energy for which $Q_{s}^{2}$ becomes equal to $2 \omega / \lambda$ and obeys $\omega_{*} \gg \omega_{0}$ for $L \gg \lambda$.

To summarize, the DLA phase-space is defined as the range (4.38) in $p_{\perp}^{2}$ together with the energy range at $\omega_{0} \ll \omega \ll \omega_{c}$. The whole analysis becomes more transparent if, instead of the original variables $\omega$ and $p_{\perp}^{2}$, one uses the variables $\tau \equiv 2 \omega / p_{\perp}^{2}$ (the lifetime of the fluctuation) and $p_{\perp}^{2}$. As an intermediate step, notice that eq. (4.38) is tantamount to

$$
\max \left(\lambda, \frac{2 \omega}{Q_{s}^{2}}\right) \ll \tau \ll \tau_{\mathrm{br}}(\omega) \equiv \frac{2 \omega}{k_{\mathrm{br}}^{2}(\omega)}=\sqrt{\frac{\omega}{\hat{q}}} .
$$

Then it is easy to see that, in terms of the new variables $\tau$ and $p_{\perp}^{2}$, the DLA phase-space can be simply characterized as (see also figure 3 for an illustration)

$$
\lambda \ll \tau \ll L, \quad 2 \hat{q} \tau \ll p_{\perp}^{2} \ll Q_{s}^{2} .
$$

Then the first order correction is computed as (compare to eq. (4.37))

$$
\delta \hat{q}^{(1)}=\bar{\alpha} \hat{q}^{(0)} \int_{\lambda}^{L} \frac{\mathrm{d} \tau}{\tau} \int_{2 \hat{q} \tau}^{Q_{s}^{2}} \frac{\mathrm{d} p_{\perp}^{2}}{p_{\perp}^{2}}=\bar{\alpha} \hat{q}^{(0)} \int_{\lambda}^{L} \frac{\mathrm{d} \tau}{\tau} \ln \frac{L}{2 \tau} \simeq \hat{q}^{(0)} \frac{\bar{\alpha}}{2} \ln ^{2} \frac{L}{\lambda},
$$


where we have used $Q_{s}^{2}=\hat{q} L$. (As before, we ignore the difference between $\hat{q}$ and $\hat{q}^{(0)}$, or the factors of 2 , in the argument of the logarithm.) The above result, which is in agreement with refs. $[9,71]$, is not the same as the result of evaluating eq. (4.37) at the minimal value of the energy $\omega=\omega_{0}$. The last calculation would be naive, in that it would incorrectly treat the contribution of the low energy region at $\omega_{0}<\omega<\omega_{*}$.

More generally, it is preferable to use the new variables $\tau$ and $p_{\perp}^{2}$ also within the DLA equation (4.35) and to replace the latter by an integral equation, where all the integration limits are explicit:

$$
\hat{q}_{L}\left(Q_{s}^{2}\right)=\hat{q}^{(0)}+\bar{\alpha} \int_{\lambda}^{L} \frac{\mathrm{d} \tau}{\tau} \int_{\hat{q} \tau}^{Q_{s}^{2}} \frac{\mathrm{d} p_{\perp}^{2}}{p_{\perp}^{2}} \hat{q}_{\tau}\left(p_{\perp}^{2}\right) .
$$

eq. (4.42) shows that the physical quantity $\hat{q}_{L}\left(Q_{s}^{2}\right)$ of interest - the renormalized jet quenching parameter as obtained after including the radiative corrections to DLA accuracy - is obtained as the value of a function of two variables, $\hat{q}_{\tau}\left(p_{\perp}^{2}\right)$, at the physical point $\tau=L$ and $p_{\perp}^{2}=Q_{s}^{2}(L)$ in the phase-space. (As it will be explained in section 4.4, this physical point lies on the saturation line for the gluon distribution of the medium; see also figure 3.) In turn, the function $\hat{q}_{\tau}\left(p_{\perp}^{2}\right)$ has support at $p_{\perp}^{2}>\hat{q} \tau$ and is obtained as the solution to the following integral equation:

$$
\hat{q}_{\tau}\left(p_{\perp}^{2}\right)=\hat{q}^{(0)}+\bar{\alpha} \int_{\lambda}^{\tau} \frac{\mathrm{d} \tau_{1}}{\tau_{1}} \int_{\hat{q} \tau_{1}}^{p_{\perp}^{2}} \frac{\mathrm{d} k_{\perp}^{2}}{k_{\perp}^{2}} \hat{q}_{\tau_{1}}\left(k_{\perp}^{2}\right) .
$$

As already stressed after eq. (4.35), the above equation differs from the standard DLA equation which appears e.g. in studies of the jet evolution in the vacuum $[17,18]$ via the $\tau$-dependence of the lower limit in the integral over $p_{\perp}^{2}$, which comes from the restriction to single scattering.

Eq. (4.43) can be easily solved via iterations. The first iteration (with $\hat{q}^{(0)}$ assumed to be scale-independent, once again) gives

$$
\delta \hat{q}_{\mathcal{\tau}}^{(1)}\left(p_{\perp}^{2}\right)=\hat{q}^{(0)} \frac{\bar{\alpha}}{2}\left(\ln ^{2} \frac{p_{\perp}^{2}}{\hat{q} \lambda}-\ln ^{2} \frac{p_{\perp}^{2}}{\hat{q} \tau}\right)
$$

which on the physical point $\tau=L$ and $p_{\perp}^{2}=\hat{q} L$ reduces to eq. (4.41), as it should. The second iteration yields (we only show its result at the physical point)

$$
\delta \hat{q}^{(2)}=\hat{q}^{(0)} \frac{\bar{\alpha}^{2}}{2 ! 3 !} \ln ^{4} \frac{L}{\lambda} .
$$

These and the subsequent terms in this iterative procedure are recognized as the Taylor expansion of the modified Bessel function ${ }^{14} \mathrm{I}_{1}(x)$ :

$$
\hat{q}_{L}\left(Q_{s}^{2}\right)=\hat{q}^{(0)} \frac{1}{\sqrt{\bar{\alpha}} \ln (L / \lambda)} \mathrm{I}_{1}\left(2 \sqrt{\bar{\alpha}} \ln \frac{L}{\lambda}\right) .
$$

The same result has been obtained in ref. [9] via a resummation of the relevant Feynman graphs. This resummation becomes pertinent when the medium is large enough, such

\footnotetext{
${ }^{14}$ This should be contrasted to the standard DLA solution, which involves the Bessel function $\mathrm{I}_{0}(x)[18]$.
} 
that $\bar{\alpha} \ln ^{2}(L / \lambda) \gtrsim 1$. In such a case, the radiative corrections enhance the medium-size dependence of the (renormalized) jet quenching parameter, which thus becomes even more non-local than it was at tree-level.

We conclude this subsection with a few comments on the physical meaning of the radiative corrections displayed in eqs. (4.41) or (4.46). As obvious from the previous calculations, these corrections are generated by the emission of soft gluons with energies $\omega$ deeply within the range between $\omega_{0}=\hat{q} \lambda^{2}$ and $\omega_{c}=\hat{q} L^{2}$ and with transverse momenta $p_{\perp}$ deeply between $k_{\mathrm{br}}(\omega)$ and $Q_{s}$. Such gluons have lifetimes considerably smaller than the medium longitudinal size $L$ and transverse sizes which are considerably larger than the size $r \sim 1 / Q_{s}$ of the original dipole. This hierarchy is furthermore respected by the successive emissions which are summed up by eq. (4.46) and whose energies are softer and softer with increasing generation. Because of this hierarchy, the corrections appear to be quasi-local on the longitudinal scale relevant for measuring the transverse momentum broadening, which is $L$. Similarly, they do not affect the transverse resolution on which we scrutinize the medium properties, which is set by $Q_{s}$. This ultimately explains why such corrections can be simply accounted for by a renormalization of the jet quenching parameter $\hat{q}_{L}\left(Q_{s}^{2}\right)$.

\subsubsection{The phase-space for the high-energy evolution}

In the previous section, we have argued that the phase-space for the double-logarithmic approximation is given by eq. (4.40), where the lower limit $\lambda$ has not yet been specified. In this subsection, we shall first explain the physical origin and the value of $\lambda$, thus following a discussion in ref. [9]. Then we shall critically revisit the original arguments in ref. [9] and demonstrate that, in general, the structure of the DLA phase-space is more complicated than suggested there (and shown in eq. (4.40)). The differences are unessential in the limit where the medium size $L$ is arbitrarily large, but they become important for realistic values of $L$, in which case they limit the validity of the DLA. Based on such considerations, we shall derive the constraint (4.52) for the applicability of the DLA (and, more generally, of the present high-energy approximations) in the case where the target is a weakly coupled QGP.

As discussed in ref. [9], the existence of a lower limit $\lambda$ on the lifetime $\tau$ of the fluctuations follows from energy-momentum conservation. In the high-energy approximation of interest, the gluon fluctuation is nearly on-shell, so it carries a 'minus' momentum $p^{-}=p_{\perp}^{2} / 2 \omega$ (recall that $\omega \equiv p^{+}$). This component cannot be inherited from the parent quark, which is a right mover, so it must be acquired via interactions with the medium. Since moreover we assume that there is only one scattering during the fluctuation, it is clear that either the virtual gluon, or its parent quark, must have absorbed a quanta having this momentum $p^{-}$. This quanta is a (generally off-shell) gluon exchanged between the projectile and some constituent of the medium - say, a thermal quark or gluon, in the case where the medium is a finite temperature plasma. Let us denote by $k^{-}$the respective 4-momentum component of that particular constituent and introduce the longitudinal momentum fraction $x \equiv p^{-} / k^{-}$, with $x \leq 1$ of course. (This is 'longitudinal' since the medium is a left mover.) We have

$$
x=\frac{p_{\perp}^{2}}{2 \omega k^{-}}=\frac{p_{\perp}^{2}}{2 p \cdot k},
$$




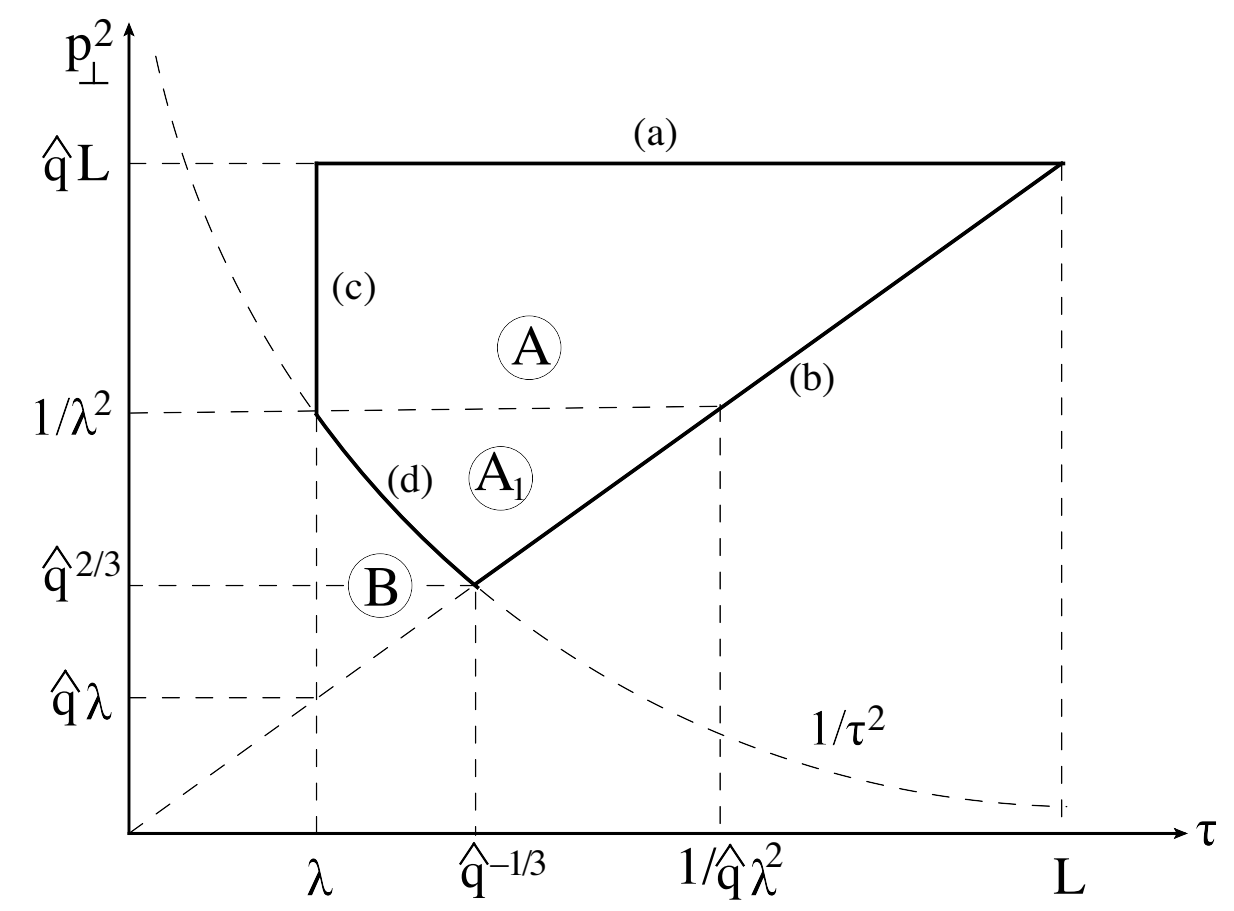

Figure 3. The phase-space for the high-energy evolution of jet quenching, in terms of the variables $\tau$ and $p_{\perp}^{2}$ (the lifetime and the transverse momentum squared of the gluon fluctuations). We assume $\hat{q} \lambda^{3} \ll 1$ and $\hat{q} L \lambda^{2} \gg 1$. Line (b) is the 'saturation line' $p_{\perp}^{2}=\hat{q} \tau$ (see section 4.4). Line (d) reads $p_{\perp}^{2}=1 / \tau^{2}$ and implements the kinematic constraint $p^{+}>p_{\perp}$. The phase-space for the doublelogarithmic evolution is region A, as delimited by the lines (a), (b), (c), and (d). For discussions of regions $\mathrm{B}$ and $\mathrm{A}_{1}$, see the main text.

where the second equality, which confirms that $x$ is boost invariant, follows from the high energy kinematics. Indeed, in whatever frame we use, at least one of the two subsequent statements is correct: (I) $p^{+}$is the large component of the 4-momentum of the gluon fluctuation, and (II) $k^{-}$is the large component of the 4-momentum of the medium constituent.

In particular, in the plasma rest frame, one has $k^{-} \simeq T$ for a typical plasma particle and eq. (4.47) becomes

$$
x \simeq \frac{p_{\perp}^{2}}{2 \omega T}=\frac{\lambda}{\tau},
$$

where $\tau=2 \omega / p_{\perp}^{2}$ and $\lambda \equiv 1 / T$ is the thermal wavelength. Since $x \leq 1$ and $\tau \leq L$, the above relation implies the following ranges of values for $x$ and $\tau$ :

$$
\frac{\lambda}{L} \leq x \leq 1, \quad L \geq \tau \geq \lambda
$$

This motivated the authors of ref. [9] to choose $\lambda=1 / T$ as the minimal value for $\tau$ in equations like (4.42). This conclusion is essentially correct, but its validity is restricted by an addition kinematical constraint, that has been overlooked in the analysis in ref. [9] and that we shall now discuss. 
The high-energy picture that we have developed so far is based on the assumption that the gluon fluctuations are very energetic in the plasma rest frame, meaning that their 'energy' $\omega$ is (much) larger than their transverse momentum: $\omega \gg p_{\perp}$. In particular, this must be true for the hardest allowed fluctuations, with energy $\sim \omega_{c}$ and transverse momentum $\sim Q_{s}$. Hence, the inequality $\omega_{c} \gg Q_{s}$, or equivalently $\hat{q} L^{3} \gg 1$, is a necessary condition for the validity of our approach. This condition has been implicitly assumed throughout our analysis and is indeed well satisfied in practice. Returning to generic values for $\omega$ and $p_{\perp}$, we observe that the kinematical constraint $\omega \gg p_{\perp}$ implies the following conditions on the lifetime $\tau=2 \omega / p_{\perp}^{2}$ of the fluctuations: $\tau \gg 1 / p_{\perp} \gg 1 / \omega$. For a given $\tau$, the transverse momentum cannot be smaller than a value $p_{\perp}^{\min } \sim \sqrt{\hat{q} \tau}$ introduced by multiple scattering. So, clearly, the kinematical constraint $\tau>1 / p_{\perp}$ is satisfied for any permitted value of $p_{\perp}$ provided the following condition is fulfilled:

$$
\tau \gtrsim \frac{1}{p_{\perp}^{\min }} \sim \frac{1}{\sqrt{\hat{q} \tau}} \Longrightarrow \tau \gtrsim \hat{q}^{-1 / 3}
$$

If this condition was satisfied for any $\tau$ within the range $\lambda \lesssim \tau<L$, then the kinematical constraint would play no special role for the present analysis (since automatically satisfied throughout the phase-space). But for a weakly coupled QGP and with $\lambda=1 / T$, the condition (4.50) is not satisfied for sufficiently small values $\tau \sim \lambda$ : one has indeed $\hat{q}^{1 / 3} \lambda \sim$ $\left[\bar{\alpha}^{2} \ln (1 / \bar{\alpha})\right]^{1 / 3} \ll 1$.

The solution to this problem is in fact quite simple: ${ }^{15}$ it suffices to impose the additional constraint $\tau>1 / p_{\perp}$ on the kinematical domain (4.40) for the double-logarithmic contributions. This leads to the phase-space denoted by the letter $\mathrm{A}$ in figure 3. Note that, in drawing this figure, we have chosen not only $\hat{q} L^{3} \gg 1$ (together with $\hat{q} \lambda^{3} \ll 1$, of course), but also the stronger condition $\hat{q} L \lambda^{2} \gg 1$. The reason for that should shortly become clear.

The domain A in figure 3 differs from the original phase-space in (4.40) by the domain denoted there by the letter B, whose contribution to $\delta \hat{q}$ can be computed as

$$
\delta \hat{q}_{\mathrm{B}}=\bar{\alpha} \hat{q}^{(0)} \int_{\lambda}^{\hat{q}^{-1 / 3}} \frac{\mathrm{d} \tau}{\tau} \int_{\hat{q} \tau}^{1 / \tau^{2}} \frac{\mathrm{d} p_{\perp}^{2}}{p_{\perp}^{2}}=\hat{q}^{(0)} \frac{\bar{\alpha}}{6} \ln ^{2} \frac{1}{\hat{q} \lambda^{3}} .
$$

This contribution is independent of the medium size $L$, so clearly it becomes negligible compared to the DLA result in eq. (4.41) - the contribution of the domains $A \cup B$ in figure 3 - for sufficiently large values of $L$. The precise condition is

$$
\frac{L}{\lambda} \gg \frac{1}{\hat{q} \lambda^{3}} \sim \frac{1}{\bar{\alpha}^{2} \ln (1 / \bar{\alpha})}, \quad \text { or, equivalently, } \quad Q_{s}^{2} \gg \frac{1}{\lambda^{2}} \sim T^{2},
$$

where the parametric estimates refer to the weakly coupled QGP. Vice-versa, these considerations suggest the existence of $L$-independent radiative corrections of order $\bar{\alpha} \ln ^{2}\left(1 / \bar{\alpha}^{2}\right)$, which are quite large and are not properly taken into account by our high-energy approximations.

\footnotetext{
${ }^{15} \mathrm{I}$ am grateful to $\mathrm{Al}$ Mueller for clarifying discussions on this point.
} 
Under the same assumptions as above, cf. eq. (4.52), the radiative corrections of interest for us here can be attributed to the relatively hard fluctuations, with transverse momenta $p_{\perp} \gg 1 / \lambda=T$. Indeed, the respective contribution of the softer momenta $p_{\perp} \leq 1 / \lambda$ is given by the domain $\mathrm{A}_{1}$ in figure 3 (a subdomain of region $\mathrm{A}$ ) and can be easily estimated as

$$
\delta \hat{q}_{\mathrm{A}_{1}}=\bar{\alpha} \hat{q}^{(0)} \int_{\hat{q}^{2 / 3}}^{1 / \lambda^{2}} \frac{\mathrm{d} p_{\perp}^{2}}{p_{\perp}^{2}} \int_{1 / p_{\perp}}^{p_{\perp}^{2} / \hat{q}} \frac{\mathrm{d} \tau}{\tau}=\hat{q}^{(0)} \frac{\bar{\alpha}}{3} \ln ^{2} \frac{1}{\hat{q} \lambda^{3}} .
$$

This contribution is comparable to that in eq. (4.51) and hence it is negligible under the same conditions. Moreover, these small contributions, from domains $\mathrm{B}$ and $\mathrm{A}_{1}$, are not even enhanced by a single large logarithm $\ln (L / \lambda)$, so they are irrelevant for the highenergy evolution.

To summarize, the gluon fluctuations which control the high-energy evolution of a slice of weakly-coupled QGP which is large enough (in the sense of eq. (4.52)) are characterized by large lifetimes $\tau \gg 1 / T$, large transverse momenta $p_{\perp} \gg T$, and even larger energies $\omega \gg p_{\perp}$. In particular, the phase-space for DLA can be restricted to the 'hard' region depicted as $\mathrm{A} \backslash \mathrm{A}_{1}$ (i.e. the difference betweens the domains $\mathrm{A}$ and $\mathrm{A}_{1}$ ) in figure 3.

Still with reference to figure 3 , it is easy to understand the origin of the subleading corrections, which are enhanced by only one logarithm — the 'energy' logarithm $\int(\mathrm{d} \tau / \tau)$. They are generated when crossing the borderlines for the integral over $p_{\perp}$, i.e. the lines (a) and (b) in that figure. The high- $p_{\perp}$ fluctuations above line (a) have small transverse sizes $B_{\perp} \lesssim 1 / Q_{s}$ and are correctly described by the BFKL equation (4.29), including the virtual term. One expects successive iterations of this equation to generate a non-perturbative 'anomalous dimension', that is, to modify the behavior of the dipole amplitude at small $r$ from (roughly) $r^{2}$ to $r^{2 \gamma}$, with $\gamma<1$ a number of $\mathcal{O}(1)$. Clearly, such corrections cannot be simply absorbed into a renormalization of $\hat{q}$. The low- $p_{\perp}$ fluctuations whose phase-space is located below line (b) have large transverse sizes $B_{\perp} \gtrsim 1 / \sqrt{\hat{q} \tau}$ and are sensible to multiple scattering. They are described by the fully non-linear equation (4.24), where one can use for that purpose approximations exploiting the fact that $B_{\perp} \gg r \sim 1 / Q_{s}$. Such large fluctuations will be discussed in section 4.5.

\subsection{Gluon evolution and saturation in the medium}

In this subsection, we shall develop an alternative physical picture for the high-energy evolution of jet quenching in terms of the gluon distribution in the medium. In particular, we would like to argue that the multiple scattering between the soft fluctuations and the medium can be alternatively interpreted as saturation effects in the gluon distribution at small $x$. A similar picture has been developed in ref. [45] for a weakly coupled QGP and in refs. [72-74] for a strongly coupled plasma described by $\mathcal{N}=4$ SYM. Here, however, we shall differ from ref. [45] in the treatment of the high-energy evolution of the saturation momentum (see below).

To develop this new picture, we will have to use a different Lorentz frame, namely, an 'infinite momentum frame' for the medium, in which the relevant gluon fluctuations appear as 'partons' from the plasma. Namely, consider the interaction between the projectile (the quark, or the dipole) and the medium (plasma) in a frame where the projectile is quite 
slow whereas the target is an ultrarelativistic left mover, with a Lorentz boost factor of order $^{16} \gamma \simeq \omega_{c} / Q_{s}=\sqrt{\hat{q} L^{3}}$. In this frame, all the fluctuations that we have discussed so far become left movers, so they are more naturally associated with the plasma. They cannot be a part of the thermal distribution, since that was not true in the plasma rest frame and the thermal distribution is boost invariant. Rather, they must be considered as bremsstrahlung (or Weizsäcker-Williams) quanta emitted by the medium constituents (thermal quarks and gluons). Thus, in this boosted frame, the relevant fluctuations are a part of the medium gluon distribution.

The typical fluctuations carry small fractions $x \ll 1$ of the longitudinal $\left(k^{-}\right)$momenta of their parent particles (which are large, $k^{-} \simeq \gamma T$, in the boosted frame). Accordingly, they have large wavelengths $\Delta x^{+} \sim 1 /\left(x k^{-}\right) \gg 1 / k^{-}$, meaning that they overlap with many medium constituents. On the other hand, they have very short lifetimes $\Delta x^{-}=1 / p^{+} \ll \gamma / T$, which explain why they cannot thermalize. (Notice that $\gamma / T$ is the smallest time scale associated with the thermal distribution in this frame.) Furthermore, the high-energy evolution that we had previously associated with the wavefunction of the projectile can be alternatively interpreted as an evolution of the medium gluon distribution with decreasing $x$. Interestingly, this evolution is somewhat different than it would be in a shockwave: the small- $x$ gluons cannot overlap with all the color sources within a longitudinal tube throughout the target (as they do in a shockwave $[12,35,36]$ ), but only with those within a longitudinal distance $\Delta x^{+} \sim 1 /\left(x k^{-}\right)$. This is consistent with the peculiar boundaries on the phase-space for linear evolution, as discussed in the previous sections. It furthermore implies a stronger $x$-dependence of the respective saturation momentum, as we now explain.

To make contact with the previous developments, let us recall that the 'unintegrated gluon distribution' in the target - the number of gluons per unit rapidity $Y \equiv \ln (1 / x)$ and per unit transverse phase-space - is closely related to the cross-section (4.1) for transverse momentum broadening. One has indeed (see e.g. the discussion in ref. [41])

$$
x \frac{\mathrm{d} N_{\mathrm{g}}}{\mathrm{d} x \mathrm{~d}^{2} \boldsymbol{p} \mathrm{d}^{2} \boldsymbol{b}}=\frac{N_{c}^{2}-1}{4 \pi^{3}} \frac{p_{\perp}^{2}}{g^{2} C_{F}} \int_{\boldsymbol{r}} \mathrm{e}^{-\mathrm{i} \boldsymbol{p} \cdot \boldsymbol{r}} \mathcal{S}(\boldsymbol{r}),
$$

where the subscript $\mathrm{g}$ stays for 'gluon' and $\boldsymbol{b}$ denotes the position in transverse space, or 'impact parameter'. More precisely, eq. (4.54) is the gluon distribution 'unintegrated' in the transverse phase-space, but integrated in the longitudinal $\left(x^{+}\right)$direction over the whole size $L$ of the target. Since our medium is assumed to be homogeneous in both $x^{+}$and $\boldsymbol{x}$, the occupation number for gluons with 3 -momentum $\left(p^{-}, \boldsymbol{p}\right)$ is naturally estimated as

$$
f\left(p^{-}, \boldsymbol{p}\right) \equiv \frac{4 \pi^{3}}{N_{c}^{2}-1} \frac{1}{L} \frac{\mathrm{d} N_{\mathrm{g}}}{\mathrm{d} p^{-} \mathrm{d}^{2} \boldsymbol{p} \mathrm{d}^{2} \boldsymbol{b}}=\frac{p_{\perp}^{2}}{g^{2} C_{F} p^{-} L} \int_{\boldsymbol{r}} \mathrm{e}^{-\mathrm{i} \boldsymbol{p} \cdot \boldsymbol{r}} \mathcal{S}(\boldsymbol{r}) .
$$

As a simple illustration, consider the high-momentum (or low occupancy) regime, where the dipole $S$-matrix in eq. (4.54) can be evaluated in the single scattering approxi-

\footnotetext{
${ }^{16}$ In the plasma rest rame, a gluon fluctuation with energy $\omega$ and transverse momentum $k_{\perp}$ has a rapidity $\eta$ such that $\gamma \equiv \cosh \eta \simeq \omega / k_{\perp}$. Since $k_{\perp}^{2} \gtrsim k_{\mathrm{br}}^{2}=\sqrt{\hat{q} \omega}$, this implies $\gamma \lesssim\left(\omega^{3} / \hat{q}\right)^{1 / 4}$, where the upper limit reaches a maximal value $\gamma_{\max }=\omega_{c} / Q_{s}$ corresponding to $\omega=\omega_{c}$.
} 
mation. At tree-level one obtains, similarly to eq. (4.9),

$$
f_{0}\left(p^{-}, p_{\perp}\right) \simeq \frac{4 \pi \alpha_{s} n_{0}}{p^{-} p_{\perp}^{2}}
$$

Although valid in the dilute regime, this result can be used to estimate the borderline of the saturation region. Namely, the non-linear effects in the gluon distribution are expected to become important when $f \sim 1 / \bar{\alpha}[12,35,36]$. This occurs for a transverse momentum $p_{\perp}$ of the order of the saturation momentum $Q_{s}(x)$, which at tree-level is estimated as

$$
Q_{s 0}^{2}(x) \sim \frac{\alpha_{s}^{2} N_{c} n_{0}}{p^{-}} \sim \frac{\hat{q}^{(0)} \lambda}{x}
$$

where we have used $p^{-}=x k^{-}$and the second equality is written, for convenience, in the plasma rest frame, where $k^{-} \simeq 1 / \lambda$ and, parametrically, $\alpha_{s}^{2} N_{c} n_{0} \sim \hat{q}^{(0)}$. Eq. (4.57) is in agreement with the corresponding result in [45]. Using $\tau=\lambda / x$, this equation can be rewritten as $Q_{s 0}^{2}(\tau)=\hat{q}^{(0)} \tau$, which is recognized as the line (b) in figure 3 (the borderline of the multiple scattering region).

What is remarkable about the saturation momentum in eq. (4.57) is that it exhibits a strong $x$-dependence already at tree-level (unlike the corresponding scale for a shockwave, which is independent of $x$ ). Clearly, this is the consequence of the fact that the longitudinal phase-space for gluon overlapping is now the longitudinal wavelength $\Delta x^{+} \propto 1 / x$ of a gluon fluctuation, and not the width $L$ of the target as a whole. This quantity $Q_{s 0}^{2}(x)$ reaches its maximal value $Q_{s 0}^{2}=\hat{q}^{(0)} L$ for $x_{\min }=\lambda / L$. Thus, the quantity that we had conventionally dubbed 'the saturation momentum' in our previous discussion (e.g. in eq. (4.7)) is in fact the proper saturation scale for the softest ${ }^{17}$ fluctuations allowed by the size of the medium. This quantity is boost invariant, but its physical interpretation as a saturation scale holds only in a frame where the target is highly boosted.

Going beyond tree-level, it is clear that the evolution of the dipole scattering amplitude in the linear approximation, eq. (4.29), can be interpreted as the BFKL evolution of the gluon occupation number in the medium. Indeed, in the single scattering approximation, eqs. (4.55) and (4.23) imply

$$
f_{\omega}\left(p^{-}, \boldsymbol{p}\right) \simeq-\frac{p_{\perp}^{2}}{p^{-}} \int_{\boldsymbol{r}} \mathrm{e}^{-\mathrm{i} \boldsymbol{p} \cdot \boldsymbol{r}} \Gamma_{\omega}(\boldsymbol{r}) \sim \frac{1}{\bar{\alpha}} \frac{\hat{q}_{\omega}\left(p_{\perp}^{2}\right)}{p^{-} p_{\perp}^{2}},
$$

where the second, parametric, estimate holds to double-logarithmic accuracy, cf. eq. (4.34). Moreover, the effects of multiple scattering, i.e. the non-linear terms in eq. (4.24), can be interpreted as gluon saturation in the medium, as we now explain. Indeed, such effects become important when the exponent in eq. (4.24) becomes of $\mathcal{O}(1)$. Using the DLA estimate (4.58) for $f_{\omega}\left(p^{-}, \boldsymbol{p}\right)$, this condition can be recognized as the saturation condition for the gluon occupation number:

$$
1 \sim \hat{q}_{\omega}\left(1 / B_{\perp}^{2}\right) B_{\perp}^{2} \Delta t \sim \frac{\hat{q}_{\omega}\left(p_{\perp}^{2}\right)}{p_{\perp}^{2}} \frac{\lambda}{x} \sim \bar{\alpha} f_{\omega}(x, \boldsymbol{p}) .
$$

\footnotetext{
${ }^{17} \mathrm{By}$ 'softest' we here mean the smallest value of $x$, as appropriate from the viewpoint of the left-moving target; from the viewpoint of the right-moving projectile, these are rather the hardest fluctuations, with 'energy' $p^{+}=\omega_{c}$.
} 
Solving this condition for $p_{\perp}^{2}$, one finds the saturation momentum in the presence of radiative corrections (to double-logarithmic accuracy):

$$
Q_{s}^{2}(x) \sim \frac{\hat{q}(x) \lambda}{x},
$$

where $\hat{q}(x) \equiv \hat{q}_{\tau}\left(p_{\perp}^{2}\right)$ with $\tau=\lambda / x$ and $p_{\perp}^{2}=Q_{s}^{2}(x)$. That is, $\hat{q}(x)$ is the function $\hat{q}_{\tau}\left(p_{\perp}^{2}\right)$ evaluated along the saturation line. (In particular, for $x=x_{\min }=\lambda / L$, this is the physical jet quenching parameter.) In view of eq. (4.43), this can be given by the following integral representation

$$
\hat{q}(x)=\hat{q}^{(0)}+\bar{\alpha} \int_{x}^{1} \frac{\mathrm{d} x_{1}}{x_{1}} \int_{Q_{s}^{2}\left(x_{1}\right)}^{Q_{s}^{2}(x)} \frac{\mathrm{d} p_{\perp}^{2}}{p_{\perp}^{2}} \hat{q}_{x_{1}}\left(p_{\perp}^{2}\right) .
$$

Within the integration limits above, one can use the zeroth order estimate $Q_{s}^{2}(x) \simeq \hat{q}^{(0)} \lambda / x$. Note that, with decreasing $x$, both the longitudinal phase-space and the transverse phasespace in eq. (4.61) increase equally fast - that is, the both increase like $\ln (1 / x)$ - due to the rapid increase $Q_{s}^{2}(x) \sim 1 / x$ of the saturation scale. Accordingly, the above DLA calculation correctly captures the dominant radiative corrections to the evolution of $Q_{s}^{2}(x)$, unlike what happens in the case of a shockwave. (For the latter, the longitudinal phasespace increases faster than the transverse one in the approach towards saturation, hence the correct calculation of $Q_{s}^{2}(x)$ requires the full BFKL equation, and not just its DLA limit $[31,32]$.) Interestingly, a DLA calculation of the plasma saturation momentum has also been proposed in ref. [45], but merely as a heuristic extrapolation of the standard DLA approximation to the DGLAP (or BFKL) equation down to a transverse scale $p_{\perp}^{2} \sim Q_{s}^{2}(x)$, where this approximation is not really justified.

In particular, if one treats the zeroth order result $\hat{q}^{(0)}$ as a constant, one finds (cf. eq. (4.46))

$$
\hat{q}(x)=\hat{q}^{(0)} \frac{1}{\sqrt{\bar{\alpha}} \ln (1 / x)} \mathrm{I}_{1}\left(2 \sqrt{\bar{\alpha}} \ln \frac{1}{x}\right) .
$$

In the extreme limit where $2 \sqrt{\bar{\alpha}} \ln (1 / x) \gg 1$, one can use the asymptotic behavior of the modified Bessel function to deduce

$$
Q_{s}^{2}(x) \simeq \frac{Q_{0}^{2}}{x^{1+\gamma_{s}}},
$$

with $Q_{0}^{2} \equiv \hat{q}^{(0)} \lambda$ and the 'anomalous dimension' $\gamma_{s}=2 \sqrt{\bar{\alpha}}$. The overall power $\lambda_{s} \equiv 1+\gamma_{s}$ in eq. (4.63) is the medium saturation exponent within the present approximation. This is independent of the precise nature of the medium, as it is fully determined by the highenergy evolution. The radiative correction $\gamma_{s}$ looks like a strong effect since $2 \sqrt{\bar{\alpha}} \sim 1$ for $\alpha_{s} \approx 0.3$ and $N_{c}=3$. But one should keep in mind that the present approximation is strictly valid only when $\bar{\alpha} \ll 1$.

This being said, it is also interesting to notice that this perturbative result appears as a reasonable interpolation towards the corresponding result in $\mathcal{N}=4 \mathrm{SYM}$ at infinitely strong coupling $\left(g^{2} N_{c} \rightarrow \infty\right)$, as obtained in [72]. Namely, ref. [72] reported a saturation 
momentum $Q_{s}^{2}(x) \sim T^{2} / x^{2}$, where the 'saturation exponent' $\lambda_{s}=2$ can be recognized as the sum of a kinematical contribution $\lambda_{s 0}=1$, the same as in eq. (4.57), and a large 'anomalous dimension' $\gamma_{s}=1$, which is the intercept of the graviton. (At strong coupling, the unitarization occurs via multiple graviton exchanges [75].) Together, the present results and the previous ones in ref. [72] suggest a rather smooth and fast transition from a weak coupling-like behavior to a strong coupling-like with increasing $\bar{\alpha}$ (at least, in the absence of running coupling effects).

\subsection{Comments on the effects of multiple scattering}

So far, we have not attempted to explicitly evaluate the non-linear terms in eq. (4.24), which encode the effects of multiple scattering. Rather, we have used them within semiquantitative considerations in order to constrain the phase-space for the linear approximation and to develop a physical picture in terms of gluon saturation. But, clearly, it would be interesting to have a more quantitative control on these effects, e.g. in order to understand the systematics of the high-energy resummation. Ideally, one would like to isolate all the radiative corrections which are enhanced by a large energy logarithm $\ln (1 / x) \sim \ln (L / \lambda)$ and thus obtain a non-linear equation which is explicitly valid to leading logarithmic accuracy. Unfortunately, this turns out to be very hard since the non-linear effects enter eq. (4.24) via a path-integral, in which the unknown function $\Gamma_{\omega}(\boldsymbol{r})$ plays the role of the effective potential. That is, eq. (4.24) is truly a functional integro-differential equation and very little is known about how to deal with such equations in practice.

In this section, we shall perform a limited study of the non-linear terms in eq. (4.24), with two main objectives: to elucidate the systematics of the logarithmically-enhanced radiative corrections (which turns out to be very different from the case where the target is a shockwave) and to better justify the arguments in the previous sections concerning the kinematics of the fluctuations and the borderlines of the single scattering regime.

Concerning the first objective above, we would like to demonstrate the following point: for the case of an extended target, and unlike for a shockwave, the individual terms beyond the first one in the multiple scattering series - i.e. the terms describing double scattering, triple scattering etc - are not separately enhanced by a large energy logarithm. This is so because the longitudinal phase-space for multiple scattering is the lifetime of the soft gluon fluctuations, which is itself energy-dependent. This being said, the effects of multiple scattering are nevertheless important for a complete calculation at leading logarithmic accuracy, in that they provide the physical cutoff for the respective contribution of the single scattering (which would otherwise be infrared divergent).

To be specific, let us first recall the way how the energy logarithm has been generated for the single-scattering contribution. This comes via the phase-space for the three time integrations in eq. (4.27): over the emission times $t_{1}$ and $t_{2}$ and over the interaction time $t$. Namely, the integral over $t$ between $t_{1}$ and $t_{2}$ scales like $\Delta t=t_{2}-t_{1}$, that over $\Delta t$ scales like $\tau_{c o h}=2 \omega / p_{\perp}^{2}$, and the final integral over, say, $t_{2}$ scales like $L$. Altogether, there is a longitudinal phase-space $L \tau_{\text {coh }}^{2} \propto \omega^{2}$ which, when combined with the overall factor $1 / \omega^{3}$ in eq. (4.27), produces the logarithmic phase-space $\int(\mathrm{d} \omega / \omega)$ for the ensuing energy integration. Now, let us similarly consider the contribution of a double scattering. As 
compared to the previous case, there are now two interaction times to be integrated over between $t_{1}$ and $t_{2}$. This introduces an additional factor $\Delta t$, so the global result scales like $L \tau_{c o h}^{3} \propto \omega^{3}$, which spoils the logarithmic integration over $\omega$. A similar conclusion holds for the contribution of $n$ successive collisions, which scales like $L \tau_{c o h}^{n+1} \propto \omega^{n+1}$.

This should be contrasted to the case of a shockwave target, as discussed in section 3.1. There, the two integrals over $t_{1}$ and $t_{2}$ separately restrict each of the emission times to values of order $\tau_{c o h}$ around $t=0$ (the position of the shockwave). Also each scattering with the target occurs within the longitudinal extent $L$ of the latter (with $L \ll \tau_{c o h}$ in this context), so the corresponding time integral brings in a factor of $L$. Hence, an individual $n$-scattering contribution with $n \geq 1$ scales like $\tau_{c o h}^{2} L^{n} \propto \omega^{2}$ and therefore it is by itself accompanied by a large energy logarithm.

Returning to the case of an extended target, one should observe that the previous, power-counting, argument is quite formal, in that it is plagued with infrared divergences: each additional scattering brings in a factor $\tau_{c o h}=2 \omega / p_{\perp}^{2}$, which becomes singular when $p_{\perp} \rightarrow 0$. This leads to a logarithmic divergence in the single-scattering contribution, as manifest on eq. (4.35), and to even stronger, power-like, divergences in the terms with two or more scatterings. We expect such divergences to be cured by the resummation of the multiple scattering series to all orders, but in order to verify this, one needs a non-perturbative calculation of this series.

To illustrate these considerations, let us consider the first iteration of eq. (4.24). That is, we shall evaluate the r.h.s. of this equation using the tree-level approximation for the dipole $S$-matrix, eq. (4.5), together with the 'harmonic approximation' for the jet quenching parameter - meaning that we ignore the scale dependence of the latter: $\hat{q} \simeq$ const (throughout this subsection, one writes $\hat{q} \equiv \hat{q}^{(0)}$ ). The harmonic approximation is indeed important for the present purposes, since it allows us to explicitly perform the path integral in eq. (4.24), which becomes

$$
\begin{aligned}
& \mathcal{I}\left(\boldsymbol{r}_{2}, \boldsymbol{r}_{1}, \Delta t\right) \\
& \quad \equiv \int[\mathcal{D} \boldsymbol{r}] \mathrm{e}^{\mathrm{i} \frac{\omega}{2} \int_{t_{1}}^{t_{2}} \mathrm{~d} t^{\prime} \dot{\boldsymbol{r}}^{2}\left(t^{\prime}\right)} \exp \left\{-\frac{\hat{q}}{4} \int_{t_{1}}^{t_{2}} \mathrm{~d} t\left[(\boldsymbol{x}-\boldsymbol{r}(t))^{2}+(\boldsymbol{r}(t)-\boldsymbol{y})^{2}-(\boldsymbol{x}-\boldsymbol{y})^{2}\right]\right\}
\end{aligned}
$$

with boundary conditions $\boldsymbol{r}\left(t_{1}\right)=\boldsymbol{r}_{1}$ and $\boldsymbol{r}\left(t_{2}\right)=\boldsymbol{r}_{2}$. ( $\mathcal{I}$ is also a function of $\boldsymbol{x}$ and $\boldsymbol{y}$, but the respective arguments are kept implicit.) A standard calculation yields

$$
\begin{aligned}
& \mathcal{I}\left(\boldsymbol{r}_{2}, \boldsymbol{r}_{1}, \Delta t\right) \\
& =\frac{-\mathrm{i}}{2 \pi} \frac{\omega \Omega}{\sinh \Omega \Delta t} \mathrm{e}^{\frac{\hat{q}}{8} \Delta t(\boldsymbol{x}-\boldsymbol{y})^{2}} \\
& \quad \times \exp \left\{\frac{\mathrm{i}}{2} \frac{\omega \Omega}{\sinh \Omega \Delta t}\left[\left(\left(\boldsymbol{r}_{2}-\boldsymbol{R}\right)^{2}+\left(\boldsymbol{r}_{1}-\boldsymbol{R}\right)^{2}\right) \cosh \Omega \Delta t-2\left(\boldsymbol{r}_{2}-\boldsymbol{R}\right) \cdot\left(\boldsymbol{r}_{1}-\boldsymbol{R}\right)\right]\right\}
\end{aligned}
$$

where $\boldsymbol{R} \equiv(\boldsymbol{x}+\boldsymbol{y}) / 2$ and

$$
\Omega \equiv \frac{1+\mathrm{i}}{\sqrt{2}} \sqrt{\frac{\hat{q}}{\omega}}=\frac{1+\mathrm{i}}{\sqrt{2}} \frac{1}{\tau_{\mathrm{br}}(\omega)}
$$


In the limit $\hat{q} \rightarrow 0$ (no scattering), this reduces to the 'non-relativistic' propagator in the vacuum, as it should (cf. eq. (2.10)): $\mathcal{I} \rightarrow \mathcal{G}_{0}$ with

$$
\mathcal{G}_{0}\left(\boldsymbol{r}_{2}-\boldsymbol{r}_{1}, \Delta t\right)=\frac{-\mathrm{i}}{2 \pi} \frac{\omega}{\Delta t} \exp \left\{\frac{\mathrm{i} \omega\left(\boldsymbol{r}_{2}-\boldsymbol{r}_{1}\right)^{2}}{2 \Delta t}\right\} \text {. }
$$

The perturbative ('small $\hat{q}$ ') expansion of eq. (4.65), which would reconstruct the multiple scattering series, turns out to be quite tedious. However, by inspection of this equation, it is clear that such an expansion makes sense only for sufficiently small times $\Delta t \ll \tau_{\mathrm{br}}(\omega)$. In this perturbative regime at early times, the convergence in $\Delta t$ is controlled by the free propagator (4.67), which implies that the transverse size of the gluon fluctuation grows via quantum diffusion: $\left|\boldsymbol{r}_{2}-\boldsymbol{r}_{1}\right| \simeq \sqrt{2 \Delta t / \omega}$. However, when $\Delta t$ approaches $\tau_{\mathrm{br}}(\omega)$, the effects of the interactions become non-perturbative. Via the Gaussian implicit ${ }^{18}$ in eq. (4.65), they restrict the further growth of the transverse separation $B_{\perp} \equiv \max \left(\left|\boldsymbol{r}_{2}-\boldsymbol{R}\right|,\left|\boldsymbol{r}_{1}-\boldsymbol{R}\right|\right)$ to values $B_{\perp} \lesssim 2 / k_{\mathrm{br}}(\omega)$, as anticipated below eq. (4.31). For even larger time separations $\Delta t \gtrsim \tau_{\text {br }}(\omega)$, we can use

$$
\frac{1}{2 \sinh \Omega \Delta t} \simeq \mathrm{e}^{-\Omega \Delta t} \propto \mathrm{e}^{-\Delta t / \sqrt{2} \tau_{\mathrm{br}}}
$$

showing that the long-lived gluon fluctuations are exponentially suppressed.

To summarize, the effect of multiple scattering is to limit the lifetime and the transverse size of a gluon fluctuation with energy $\omega$ to values $\Delta t \lesssim \tau_{\text {br }}(\omega)$ and respectively $B_{\perp} \lesssim$ $2 / k_{\mathrm{br}}(\omega)$. These values are as expected: for them, the exponent in eq. (4.24), i.e. the amplitude for a single scattering during the lifetime of the fluctuation, becomes of $\mathcal{O}(1)$. Moreover, since the perturbative expansion of eq. (4.65) is truly an expansion in powers of $\Delta t / \tau_{\mathrm{br}}(\omega)$ and $B_{\perp} k_{\mathrm{br}}(\omega)$, it is clear that the single scattering approximation - which corresponds to the first non-trivial term in this expansion - is valid only for fluctuations which are hard enough (in the sense of having a sufficiently large transverse momentum) for $\Delta t \ll \tau_{\mathrm{br}}(\omega)$ and $B_{\perp} \ll 1 / k_{\mathrm{br}}(\omega)$. These conditions have been often used in the previous discussion in this section.

Using the explicit expression for the path integral in eq. (4.65), it is in principle possible the fully evaluate the r.h.s. of eq. (4.24) and thus compute the leading-order radiative correction to the dipole amplitude beyond the double-logarithmic approximation. In general this calculation is hindered by the complexity of the time integrations. In ref. [9], this calculation has been pushed to single logarithmic accuracy — that is, one has explicitly evaluated the subleading correction to $\hat{q}$ of order $\bar{\alpha} \ln (L / \lambda)$. We shall not repeat here the manipulations in ref. [9], but merely explain how the logarithmic enhancement at small $\omega$ emerges in the regime controlled by multiple scattering, i.e. for the relatively large fluctuations with transverse size $B_{\perp} \sim 1 / k_{\mathrm{br}}(\omega)$. In this regime, the integral over $t_{2}-t_{1}$ is cut off by the exponential in eq. (4.68), hence the two integrals over $t_{1}$ and $t_{2}$ give a factor $L \tau_{\mathrm{br}}$. This is multiplied by ( $i$ ) the overall factor $1 / \omega^{3}$ in the r.h.s. of eq. (4.24), (ii) the factor $\omega \Omega \sim \omega / \tau_{\mathrm{br}}$ explicit in the prefactor of eq. (4.65), and (iii) a factor $k_{\mathrm{br}}^{4}=4 \hat{q} \omega$ which is

\footnotetext{
${ }^{18}$ Notice that the complex exponential in the second line of eq. (4.65) is proportional to $\exp \left\{-\omega B_{\perp}^{2} / \sqrt{2} \tau_{\mathrm{br}}(\omega)\right\}$.
} 
generated as follows: the integrand of eq. (4.24) involves two transverse derivatives acting on $\mathcal{I}\left(\boldsymbol{r}_{2}, \boldsymbol{r}_{1}, \Delta t\right)$, which bring down a factor $k_{\mathrm{br}}^{2}$. Moreover, the r.h.s. of eq. (4.24) vanishes when $\boldsymbol{r}=\boldsymbol{x}-\boldsymbol{y} \rightarrow 0$ ('color transparency') and in the regime of multiple scattering it can be evaluated to leading order in the expansion in powers of $\boldsymbol{r}^{2}$. (Such an expansion is indeed legitimate since $r \sim 1 / Q_{s} \ll B_{\perp}$.) This expansion introduces two additional transverse derivatives, hence another factor of $k_{\mathrm{br}}^{2}$. Altogether, one generates a factor $1 / \omega$, synonimuous of logarithmic enhancement. Incidentally, the above arguments also show that the contribution of multiple scattering to the evolution equation for the scattering amplitude per unit time reads, parametrically,

$$
g^{2} C_{F} \omega \frac{\partial \Gamma_{\omega}(\boldsymbol{r})}{\partial \omega} \sim \bar{\alpha} \hat{q} \boldsymbol{r}^{2}
$$

This has the right structure to be interpreted as a correction to the jet quenching parameter (compare to eq. (4.34)). However, this is likely not the case for the single-logarithmic corrections coming from the opposite limit, namely from the very small fluctuations with sizes $B_{\perp} \sim 1 / Q_{s}$, as described by the BFKL equation (4.29).

\section{The high-energy evolution of the radiative energy loss}

In the previous section, we have studied the high-energy evolution of the transverse momentum broadening for an energetic quark propagating through a dense QCD medium. As well known, this physical problem is closely related to another one: the energy loss by an energetic parton via medium-induced radiation, that is, gluon emissions which are triggered by the interactions between the parent parton or the radiated gluon and the constituents of the medium. Within the high-energy kinematics of interest, the differential cross-section for such an emission involves Wilson line correlators which measure the color coherence between the emitter and its radiation. This coherence is progressively washed out via rescattering in the medium and thus is sensible to the physics of collisions, as encoded in $\hat{q}$. This relation is manifest at tree level, where any (gauge-invariant) Wilson line correlator can be expressed in terms of the 'dipole cross-section' (the exponent in eq. (4.4)) and hence in terms of $\hat{q}^{(0)}$, eq. (4.6). In what follows, we would like to demonstrate that this relation remains valid after including the effects of the high-energy evolution within the double-logarithmic approximation. That is, in order to compute the radiative energy loss in the presence of radiative corrections and to DLA accuracy, one can use the same formulæ as at tree level, but with $\hat{q}^{(0)}$ replaced by the solution $\hat{q}_{L}\left(Q_{s}^{2}\right)$ to eq. (4.42). A similar conclusion has been obtained in refs. $[15,16]$, where the evolution equation (4.42) for $\hat{q}$ has been inferred via a diagrammatic argument, namely, by explicitly computing loop corrections to the radiative energy loss in the double-logarithmic approximation.

\subsection{The tree-level approximation: the BDMPSZ formalism}

To start with, let us briefly review the relevant formalism at tree-level, namely the BDMPSZ calculation of medium-induced gluon radiation [46-56]. 
We consider the emission of a single gluon by an asymptotic quark and assume, for simplicity, that the incoming quark is energetic enough to be treated in the eikonal approximation. On the other hand, the eikonal approximation cannot be used for the emitted gluon, because the transverse diffusion plays an essential role for the gluon formation. The energy lost by the quark is the energy taken away by the emitted gluon and can be computed from the respective spectrum as

$$
\Delta E=\int_{0}^{\omega_{c}} \mathrm{~d} k^{+} k^{+} \frac{\mathrm{d} N_{g}}{\mathrm{~d} k^{+}}=\int_{0}^{\omega_{c}} \mathrm{~d} k^{+} \int \mathrm{d}^{2} \boldsymbol{k} k^{+} \frac{\mathrm{d} N_{g}}{\mathrm{~d} k^{+} \mathrm{d}^{2} \boldsymbol{k}} .
$$

The upper cutoff $\omega_{c}$ stems from the fact that only gluons with energies $k^{+}<\omega_{c}$ can be emitted via the mechanism at hand (see below). Moreover, the spectrum $k^{+}\left(\mathrm{d} N_{g} / \mathrm{d} k^{+}\right)$ of the radiated gluons is such that the integral over $k^{+}$in eq. (5.1) is dominated by this upper cutoff. Accordingly, in what follows we shall focus on the emission of relatively hard gluons, with $k^{+} \sim \omega_{c}$.

As before, we assume that the medium correlations at tree-level are Gaussian and local in $x^{+}$. Under these assumptions, one can deduce the following formula for the spectrum of the medium-induced gluon radiation [52] (see also refs. [1, 4, 5, 76, 77] for pedagogical discussions)

$$
\begin{aligned}
k^{+} \frac{\mathrm{d} N_{g}}{\mathrm{~d} k^{+} \mathrm{d}^{2} \boldsymbol{k}}= & \frac{\alpha_{s} C_{F}}{2 \pi^{2}} \frac{1}{\left(k^{+}\right)^{2}} \operatorname{Re} \int_{-\infty}^{\infty} \mathrm{d} x^{+} \int_{-\infty}^{x^{+}} \mathrm{d} y^{+} \\
& \times\left.\int \mathrm{d}^{2} \boldsymbol{x} \mathrm{e}^{-\mathrm{i} \boldsymbol{k} \cdot \boldsymbol{x}} \mathcal{S}_{L, x^{+}}^{\mathrm{adj}}(\boldsymbol{x}) \partial_{\boldsymbol{x}}^{i} \partial_{\boldsymbol{y}}^{i} \mathcal{K}\left(x^{+}, \boldsymbol{x} ; y^{+}, \boldsymbol{y} ; k^{+}\right)\right|_{\boldsymbol{y}=0} .
\end{aligned}
$$

As announced, we consider an on-shell (or 'asymptotic') quark which enters the medium coming from far away. ${ }^{19}$ eq. (5.2) is a cross-section, so it is obtained by multiplying the direct amplitude (DA) times the complex conjugate amplitude (CCA), as illustrated in figure 4 . The time variables $y^{+}$and $x^{+}$are the emission times in the DA and respectively the CCA, so their difference $\Delta x^{+}=x^{+}-y^{+}$is indicative of the formation time. We have chosen $x^{+}>y^{+}$and multiplied the result by 2 to account for the opposite time ordering. Also, $\boldsymbol{x}_{0}=0$ is the transverse position of the quark, which remains unchanged during the process (eikonal approximation) and is the same in the DA and the CCA (since we do not measure the transverse momentum broadening of the quark). In writing eq. (5.2), we have already set $\boldsymbol{x}_{0}=0$, but in the subsequent discussion we shall keep the notation $\boldsymbol{x}_{0}$ at intermediate stages, for more clarity. On the other hand, the transverse momentum of the emitted gluon is measured, so the respective transverse coordinates are different in the DA and the CCA. Their difference is denoted as $\boldsymbol{x}$ in eq. (5.2) and it is conjugated to the transverse momentum $\boldsymbol{k}$ via the Fourier transform. With these differences in mind, eq. (5.2) is quite similar to eq. (4.22) for the emission of a virtual gluon and can be read by analogy with the latter.

Once again, the locality of the medium correlations in $x^{+}$has allowed us to factorize the process into three stages, like in eq. (4.15), and thus express the cross-section for

\footnotetext{
${ }^{19}$ The case of an off-shell quark which is produced by a hard process occurring at some finite time $x_{0}^{+}$ can be obtained be replacing $-\infty \rightarrow x_{0}^{+}$in the lower limits of the time integrations in eq. (5.2).
} 


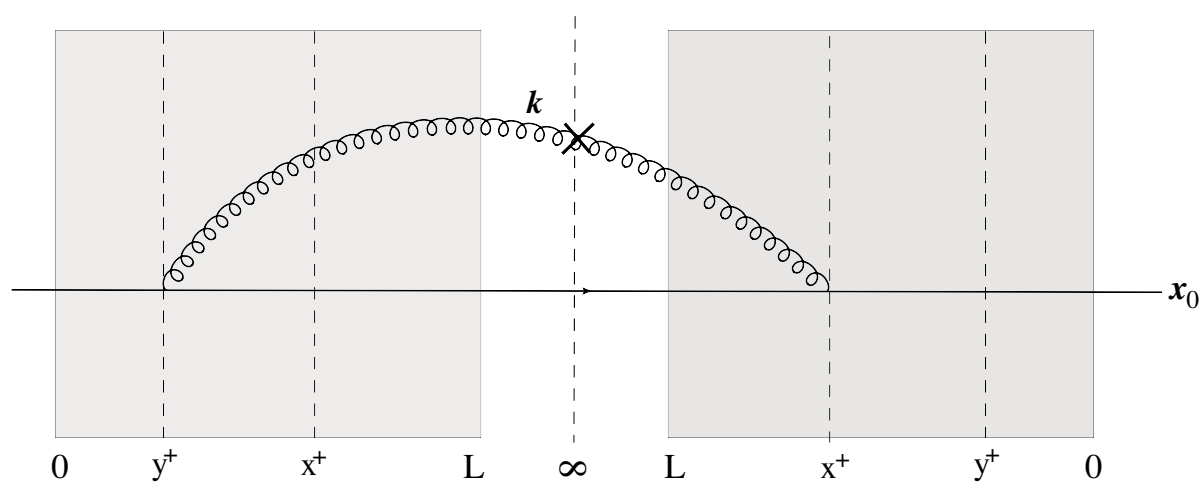

Figure 4. Feynman graph contributing to the cross-section for producing a gluon with 3-momentum $k=\left(k^{+}, \boldsymbol{k}\right)$, as computed in eq. (5.2). The l.h.s. corresponds to the DA and the r.h.s. to the CCA. Both emissions times $y^{+}$and $x^{+}$are chosen inside the medium, $0<y^{+}<x^{+}<L$, since this is the most interesting configuration for our present purposes.

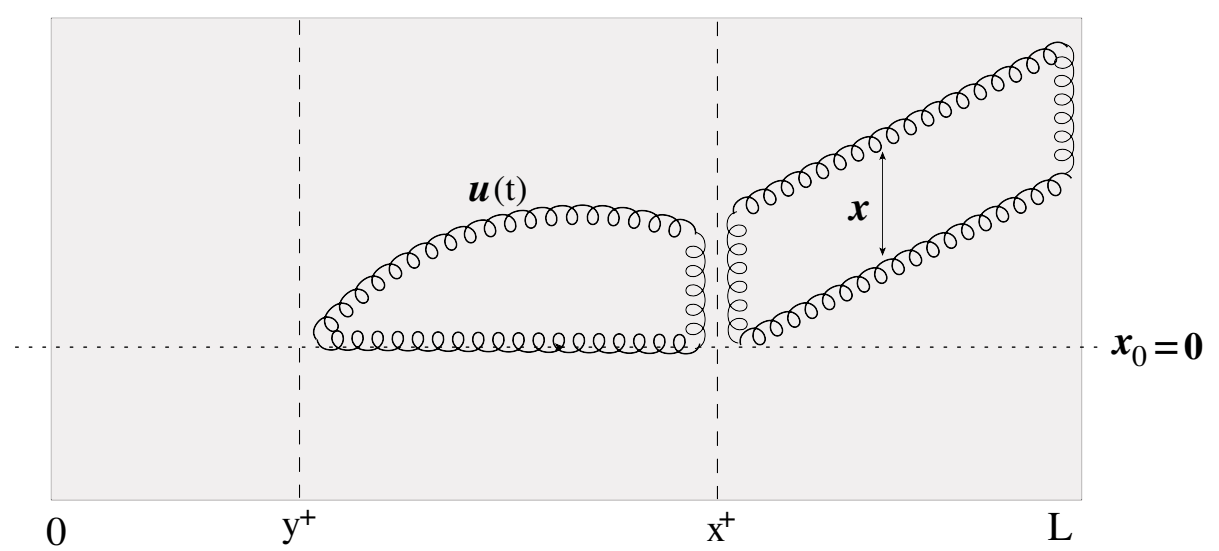

Figure 5. Alternative representation for the cross-section in figure 4, in terms of dipole amplitudes, which is obtained after performing the medium average in the Gaussian approximation. The 'vertical' lines closing the two dipoles represent the sum over the color indices.

gluon radiation in terms of scattering amplitudes for effective dipoles (see figure 5 for an illustration of this representation):

(i) Prior to the first gluon emission, i.e. for time values smaller than $y^{+}$: the Wilson lines describing the color precession of the quark mutually cancel between the DA and the CCA, by unitarity. Accordingly, there is no imprint of this first stage on the cross-section in eq. (5.2).

(ii) During the formation time, i.e. for time values between $y^{+}$and $x^{+}$: the partonic system consists in a quark-gluon pair in the DA and the original quark in the CCA. The relevant Wilson line correlator reads

$$
\begin{aligned}
\left\langle U_{x^{+} y^{+}}^{\dagger a}[\boldsymbol{u}] \frac{\operatorname{tr}}{N_{c}}\left(t^{a} V_{x^{+} y^{+}}^{\dagger}\left(\boldsymbol{x}_{0}\right) t^{b} V_{x^{+} y^{+}}\left(\boldsymbol{x}_{0}\right)\right)\right\rangle & =\frac{1}{2 N_{c}}\left\langle\operatorname{Tr} U_{x^{+} y^{+}}^{\dagger}[\boldsymbol{u}] U_{x^{+} y^{+}}\left(\boldsymbol{x}_{0}\right)\right\rangle \\
& =C_{F} \mathcal{S}_{x^{+}, y^{+}}^{\text {adj }}\left([\boldsymbol{u}], \boldsymbol{x}_{0}\right),
\end{aligned}
$$


where $t^{a}$ and $t^{b}$ are color matrices at the emission vertices, $\boldsymbol{u}(t)$ represents the trajectory of the gluon in the DA for times $y^{+} \leq t \leq x^{+}$, and $\mathcal{S}^{\text {adj }}$ is the average $S$-matrix for a color dipole in the adjoint representation,

$$
\mathcal{S}_{x^{+}, y^{+}}^{\mathrm{adj}}(\boldsymbol{x}, \boldsymbol{y}) \equiv \frac{1}{N_{c}^{2}-1}\left\langle\operatorname{Tr} U_{x^{+} y^{+}}^{\dagger}(\boldsymbol{x}) U_{x^{+} y^{+}}(\boldsymbol{y})\right\rangle .
$$

The dipole in eq. (5.3) is built with one adjoint Wilson line for the emitted gluon and another one for the precession of the color current of the quark (a color vector in the adjoint representation). The 'dipole propagator' $\mathcal{K}\left(x^{+}, \boldsymbol{x} ; y^{+}, \boldsymbol{y} ; k^{+}\right)$which enters eq. (5.2) represents the functional average of the Wilson line correlator (5.3) over the quantum trajectories of the gluon:

$$
\mathcal{K}\left(x^{+}, \boldsymbol{x} ; y^{+}, \boldsymbol{y} ; k^{+}\right)=\int[\mathcal{D} \boldsymbol{u}] \mathrm{e}^{i \frac{k^{+}}{2} \int_{y^{+}}^{x^{+}} \mathrm{d} t \dot{\boldsymbol{u}}^{2}(t)} \mathcal{S}_{x^{+}, y^{+}}^{\mathrm{adj}}\left([\boldsymbol{u}(t)], \boldsymbol{x}_{0}\right),
$$

with boundary conditions $\boldsymbol{u}\left(y^{+}\right)=\boldsymbol{y}$ and $\boldsymbol{u}\left(x^{+}\right)=\boldsymbol{x}$.

(iii) After the gluon formation, i.e. for time values larger than $x^{+}$: in this case too, the quark Wilson lines from the DA and the CCA mutually cancel, so we are left with two adjoint Wilson lines, which both refer to the emitted gluon (one for the DA, the other one for the CCA). These Wilson lines combine in the adjoint dipole $\mathcal{S}_{L, x^{+}}^{\text {adj }}\left(\boldsymbol{x}-\boldsymbol{x}_{0}\right)$, which describes the transverse momentum broadening acquired by the gluon after formation (so long as $x^{+}<L$, of course). The average transverse size of this dipole is constant, due to the medium homogeneity in the transverse plane, and hence it is equal to its original value at time $x^{+}$, which is $\boldsymbol{x}-\boldsymbol{x}_{0}=\boldsymbol{x}$.

If one is not interested in the $k_{\perp}$-spectrum of the produced gluon, but only in the energy lost by the quark, then one can integrate eq. (5.2) over $\boldsymbol{k}$ and use $\mathcal{S}_{L, x^{+}}^{\text {adj }}(0)=1$, to deduce

$$
k^{+} \frac{\mathrm{d} N_{g}}{\mathrm{~d} k^{+}}=\left.\frac{2 \alpha_{s} C_{F}}{\left(k^{+}\right)^{2}} \operatorname{Re} \int_{-\infty}^{\infty} \mathrm{d} x^{+} \int_{-\infty}^{x^{+}} \mathrm{d} y^{+} \partial_{\boldsymbol{x}}^{i} \partial_{\boldsymbol{y}}^{i} \mathcal{K}\left(x^{+}, \boldsymbol{x} ; y^{+}, \boldsymbol{y} ; k^{+}\right)\right|_{\boldsymbol{x}=\boldsymbol{y}=0}
$$

To be more specific, consider the situation where the emission occurs within the medium in both the DA and the CCA: $0<y^{+}<x^{+}<L$. This situation yields the dominant contribution for sufficiently small energies $k^{+} \ll \omega_{c}$, but the corresponding result can also be used when $k^{+} \sim \omega_{c}$, at least for parametric estimates. Then the average dipole $S$-matrix in eq. (5.3) can be computed as in eqs. (4.4)-(4.6) and reads (we set $\boldsymbol{x}_{0}=0$ from now on)

$$
\mathcal{S}_{x^{+}, y^{+}}^{\text {adj }}[\boldsymbol{u}] \simeq \exp \left\{-\frac{1}{4} \int_{y^{+}}^{x^{+}} \mathrm{d} t \hat{q}_{\mathrm{g}}\left(1 / u^{2}\right) \boldsymbol{u}^{2}(t)\right\}, \quad \hat{q}_{\mathrm{g}}\left(Q^{2}\right) \equiv \frac{N_{c}}{C_{F}} \hat{q}\left(Q^{2}\right),
$$

where the subscript 'g' in $\hat{q}_{\mathrm{g}}$ refers to 'gluon'. (The quantity $\hat{q}\left(Q^{2}\right)$ without any subscript refers to a quark in the fundamental representation and has been introduced in eq. (4.6).)

To compute the path integral in eq. (5.5), we perform the 'harmonic approximation' in eq. (5.7), that is, we replace $\hat{q}_{\mathrm{g}}\left(1 / u^{2}\right) \rightarrow \hat{q}_{\mathrm{g}}\left(k_{\mathrm{br}}^{2}\right)$, where $k_{\mathrm{br}}^{2}\left(k^{+}\right) \equiv \sqrt{2 k^{+} \hat{q}_{\mathrm{g}}}$ is the transverse 
momentum acquired by the gluon during formation. This approximation is appropriate for gluon emissions triggered by multiple soft scattering in the medium. Then the path integral yields

$$
\mathcal{K}\left(x^{+}, \boldsymbol{x} ; y^{+}, \boldsymbol{y} ; k^{+}\right)=\frac{-\mathrm{i}}{2 \pi} \frac{k^{+} \Omega}{\sinh \Omega \Delta \tau} \exp \left\{\frac{\mathrm{i}}{2} \frac{k^{+} \Omega}{\sinh \Omega \Delta \tau}\left[\left(\boldsymbol{x}^{2}+\boldsymbol{y}^{2}\right) \cosh \Omega \Delta \tau-2 \boldsymbol{x} \cdot \boldsymbol{y}\right]\right\},
$$

where $\Delta \tau=x^{+}-y^{+}$and $^{20}$

$$
\Omega=\frac{1+\mathrm{i}}{\sqrt{2}} \frac{1}{\tau_{\mathrm{br}}\left(k^{+}\right)}, \quad \tau_{\mathrm{br}}\left(k^{+}\right)=\sqrt{\frac{2 k^{+}}{\hat{q}_{\mathrm{g}}}} .
$$

Note that, even if the values $\boldsymbol{x}$ and $\boldsymbol{y}$ of the endpoints are chosen to be small (e.g. $\boldsymbol{x}=\boldsymbol{y}=0$ in the case of eq. (5.6)), the path integral (5.5) is dominated by paths $\boldsymbol{u}(t)$ along which the dipole (5.7) is close to saturation, that is, such that the exponent in eq. (5.7) is of $\mathcal{O}(1)$. Such paths are indeed favored by the competition between the quantum phase in the integrand of (5.5), which describes transverse diffusion, and the dipole $S$-matrix, which describes multiple scattering (recall also the discussion after (4.67)). Thus the spectrum (5.6) is indeed controlled by dipole sizes in the vicinity of the saturation line, as anticipated in the Introduction.

It is now straightforward to evaluate the transverse derivatives in eq. (5.6) and then perform the time integrals within the range $0<y^{+}<x^{+}<L$. In this process, one must subtract the vacuum piece of eq. (5.8), this is, its limit when $\hat{q}_{\mathrm{g}} \rightarrow 0$ : this would give a spurious contribution, which is moreover divergent. This procedure yields the BDMPSZ spectrum for soft energies $k^{+} \ll \omega_{c}$ :

$$
k^{+} \frac{\mathrm{d} N_{g}}{\mathrm{~d} k^{+}} \simeq \frac{2 \alpha_{s} C_{F}}{\pi} \sqrt{\frac{\omega_{c}}{2 k^{+}}} \quad \text { with } \quad \omega_{c}=\frac{1}{2} \hat{q}_{\mathrm{g}} L^{2} .
$$

When inserted into eq. (5.1), this gives an energy loss scaling like $L^{2}$ :

$$
\Delta E(L)=\kappa \frac{2 \alpha_{s} C_{F}}{\pi} \omega_{c}=\kappa \frac{\alpha_{s} C_{F}}{\pi} \hat{q}_{\mathrm{g}} L^{2},
$$

with $\kappa$ a number of $\mathcal{O}(1)$. (eq. (5.10) would predict $\kappa=\sqrt{2}$ but this value changes after using the correct version of the BDMPSZ spectrum, which remains valid when $k^{+} \sim$ $\left.\omega_{c}[48,49,51].\right)$

This result can be used to check that the integral in eq. (5.1) is indeed dominated by its upper limit. The general result valid for any $k^{+}$can be found in refs. [48, 49, 51].

Concerning the $k_{\perp}$-spectrum, notice that the dominant dependence upon $\boldsymbol{x}$ within the integrand of eq. (5.2) is contained in the following product of two Gaussians:

$$
\exp \left\{\frac{\mathrm{i}}{2} k^{+} \Omega \operatorname{coth} \Omega \Delta \tau \boldsymbol{x}^{2}\right\} \exp \left\{-\frac{\hat{q}_{\mathrm{g}}}{4}\left(L-x^{+}\right) \boldsymbol{x}^{2}\right\} \text {. }
$$

\footnotetext{
${ }^{20}$ The current expressions for $\tau_{\mathrm{br}}\left(k^{+}\right)$and $k_{\mathrm{br}}\left(k^{+}\right)$are consistent with their respective definitions in section 4 in view of the relation $\hat{q}_{\mathrm{g}} \simeq 2 \hat{q}$ valid at large $N_{c}$. (Recall that the discussion in section 4 was carried mostly at large $N_{c}$.)
} 
The first factor arises after letting $\boldsymbol{y}=0$ in eq. (5.8), while the second one is the two-gluon dipole $\mathcal{S}_{L, x^{+}}^{\text {adj }}(\boldsymbol{x})$ with $\hat{q}_{\mathrm{g}}$ evaluated at a momentum scale $\sim Q_{s}^{2}$. The transverse momentum spectrum obtained via the Fourier transform of the above is clearly Gaussian and peaked at a typical value

$$
\left\langle k_{\perp}^{2}\right\rangle \simeq \sqrt{2 k^{+} \hat{q}_{\mathrm{g}}}+\hat{q}_{\mathrm{g}}\left(L-x^{+}\right) \sim Q_{s}^{2} \equiv \hat{q}_{\mathrm{g}} L
$$

where $Q_{s}^{2}$ now denotes the gluon saturation momentum. In eq. (5.13) we recognize the sum of the momentum broadening acquired via collisions during the formation time and that acquired after the formation. For $k^{+} \sim \omega_{c}$, both contributions are parametrically of $\mathcal{O}\left(Q_{s}^{2}\right)$. More details on the $k_{\perp}$-spectrum can be found in refs. [52, 53, 78].

Returning to eq. (5.8), this can be used to read the characteristic scales for gluon formation. The r.h.s. of eq. (5.8) is exponentially suppressed for time separations $\Delta \tau \gg \tau_{\mathrm{br}}$ and for transverse separations $\left(\boldsymbol{x}-\boldsymbol{x}_{0}\right)^{2} \gg 1 / k_{\mathrm{br}}^{2}$ (recall that we set $\boldsymbol{x}_{0}=0$ ). Accordingly, the emission of a gluon with energy $k^{+}$via the present mechanism takes a time of order $\tau_{\mathrm{br}}\left(k^{+}\right)$. Also, the maximal transverse separation between the emitted gluon and its parent parton is of order $1 / k_{\mathrm{br}}\left(k^{+}\right)$. When $k^{+} \sim \omega_{c}$, as relevant for the calculation of the energy loss, these scales become $\tau_{\mathrm{br}} \sim L$ and $1 / k_{\mathrm{br}} \sim 1 / Q_{s}$ - that is, they are parametrically similar to those underlying the physics of transverse momentum broadening, as discussed in section 4. Hence, no surprisingly, the respective discussions of the radiative corrections will be quite similar as well.

\subsection{The dominant radiative corrections}

Without loss of generality, we can restrict our discussion of the evolution to the case where the gluon with longitudinal momentum $k^{+} \sim \omega_{c}$ (the one which is responsible for the energy loss) is emitted inside the medium, in both the direct and the complex conjugate amplitudes. (Indeed this case is the most complicated one, in terms of medium interactions.) We keep the same conventions as before: the gluon is first emitted in the DA, at time $y^{+}$, and then in the CCA, at time $x^{+}$.

As in section 4, we assume that the high-energy evolution preserves the Gaussian nature of the medium correlations, cf. eq. (4.14). It is then quite clear that this evolution will also preserve the factorization of the cross-section into the three stages discussed in section 5.1. This is so because the relevant quantum fluctuations are short-lived: their coherence time $\tau_{c o h}=2 \omega / p_{\perp}^{2}$ is much shorter than the typical duration of any of these three stages. As before, in section 4 , the variables $\omega$ and $p_{\perp}$ denote the 'energy' (in the sense of $p^{+}$) and the transverse momentum of the evolution gluon, and the most interesting situation is such that $\omega \ll k^{+}$and $p_{\perp} \ll Q_{s}$. In this situation, the quantum fluctuations which overlap with two different stages (and thus could break down the factorization) are suppressed by the smallness of their longitudinal phase-space.

Consider e.g. a fluctuation where the soft gluon is emitted by the quark at some time $t_{1}<y^{+}$and then absorbed by either the quark or the nascent gluon at some time $t_{2}$ during the 'formation' stage $\left(y^{+}<t_{2}<x^{+}\right)$. This fluctuation has a lifetime $t_{2}-t_{1} \sim \tau_{c o h}$, so both $t_{1}$ and $t_{2}$ must lie within an interval $\sim \tau_{c o h}$ around $y^{+}$. Accordingly, the respective 
longitudinal phase-space is of order $\tau_{c o h}^{2}$ and thus is much smaller than that, of order $\left(x^{+}-y^{+}\right) \tau_{c o h}$, corresponding to fluctuations which fully develop during the formation time $\left(y^{+}<t_{1}<t_{2}<x^{+}\right)$. We have indeed $x^{+}-y^{+} \sim \tau_{\mathrm{br}}\left(k^{+}\right) \gg \tau_{c o h}(\omega)$ for $\omega \ll k^{+}$. This discussion implies that the cross-section for medium-induced radiation can still be given the factorized structure in eq. (5.2), but with the individual factors generally modified by radiative corrections.

With reference to eq. (5.2), it is quite obvious that the evolution has no influence on the first stage at $t<y^{+}$, i.e. prior to the emission of the nascent gluon in the DA. During that stage, the quarks in the DA and the CCA make up a zero-size 'dipole', which does not interact, so its high-energy evolution cannot be measured. It is furthermore clear that the main effect of the evolution during the last stage at $t>x^{+}$(after gluon formation) is to renormalize the jet quenching parameter within the two-gluon dipole amplitude $\mathcal{S}_{L, x^{+}}^{\text {adj }}(\boldsymbol{x})$, in the way explained in section 4.3.2: to double-logarithmic accuracy, the renormalized adjoint dipole $S$-matrix reads

$$
\mathcal{S}_{L, x^{+}}^{\text {adj }}(\boldsymbol{x}) \simeq \exp \left\{-\frac{1}{4} \hat{q}_{\mathrm{g}}\left(L, Q_{s}^{2}\right)\left(L-x^{+}\right) \boldsymbol{x}^{2}\right\},
$$

with $\hat{q}_{\mathrm{g}}\left(L, Q_{s}^{2}\right)$ the solution to eq. (4.42) for $Q_{s}^{2}=\hat{q}_{\mathrm{g}} L$. This differs from the corresponding quark transport coefficient merely by a color factor: $\hat{q}_{\mathrm{g}}\left(L, Q_{s}^{2}\right)=\left(N_{c} / C_{F}\right) \hat{q}_{L}\left(Q_{s}^{2}\right)$. In choosing the scales for $\hat{q}_{\mathrm{g}}$ above, we have used the fact that, parametrically, $L-x^{+} \sim L$ and $x_{\perp}^{2} \sim 1 / Q_{s}^{2}$.

As compared to section 4, the only situation which is somewhat new is when the fluctuation lives during the formation time of the radiated gluon. This is new since, unlike in section 4, we do not assume anymore the eikonal approximation for the evolving dipole: the trajectory $\boldsymbol{u}(t)$ of the nascent gluon, which is the same as the size of the effective dipole ( since the quark is fixed at $\boldsymbol{x}_{0}=0$ ), is randomly varying via transverse diffusion. Yet, this transverse motion looks relatively slow on the typical time scale for quantum fluctuations (since the respective 'transverse mass' $k^{+}$is comparatively hard), so we expect some kind of 'adiabatic approximation' to be applicable for the fluctuations. This will be detailed in what follows.

The respective evolution equation is obtained as in section 4.2, that is, by first acting with the Hamiltonian $\Delta H$ on the (adjoint) dipole scattering operator $\hat{S}_{x^{+}, y^{+}}^{\text {adj }}[\boldsymbol{u}]$ and then performing the medium average within the Gaussian approximation (4.14). This procedure implies

$$
\mathcal{S}_{x^{+}, y^{+}}^{\mathrm{adj}}([\boldsymbol{u}] ; \omega)=\exp \left\{-g^{2} N_{c} \int_{y^{+}}^{x^{+}} \mathrm{d} t \Gamma_{\omega}(\boldsymbol{u}(t))\right\}
$$

with the function $\Gamma_{\omega}(\boldsymbol{r})$ now obeying (compare to eq. (4.24))

$$
\begin{aligned}
\int_{y^{+}}^{x^{+}} \mathrm{d} t \frac{\partial \Gamma_{\omega}(\boldsymbol{u}(t))}{\partial \omega} & =\frac{1}{4 \pi \omega^{3}} \int_{y^{+}}^{x^{+}} \mathrm{d} t_{2} \int_{y^{+}}^{t_{2}} \mathrm{~d} t_{1} \partial_{\boldsymbol{r}_{1}}^{i} \partial_{\boldsymbol{r}_{2}}^{i}\left\{\int[\mathcal{D} \boldsymbol{r}] \mathrm{e}^{\mathrm{i} \frac{\omega}{2} \int_{t_{1}}^{t_{2}} \mathrm{~d} t^{\prime} \dot{\boldsymbol{r}}^{2}\left(t^{\prime}\right)}\right. \\
& \left.\times\left[\mathrm{e}^{-\frac{g^{2} N_{c}}{2} \int_{t_{1}}^{t_{2}} \mathrm{~d} t\left(\Gamma_{\omega}(\boldsymbol{u}(t)-\boldsymbol{r}(t))+\Gamma_{\omega}(\boldsymbol{r}(t))-\Gamma_{\omega}(\boldsymbol{u}(t))\right)}-1\right]\right\}\left.\left.\right|_{\boldsymbol{r}_{2}=0} ^{\boldsymbol{r}_{2}=\boldsymbol{u}\left(t_{2}\right)}\right|_{\boldsymbol{r}_{1}=0} ^{\boldsymbol{r}_{1}=\boldsymbol{u}\left(t_{1}\right)}
\end{aligned}
$$


Within the present approximations, these equations hold for arbitrary $N_{c}$. The main difference w.r.t. eq. (4.24) is the fact that the endpoints $\boldsymbol{r}_{1}$ and $\boldsymbol{r}_{2}$ of the path integral in eq. (5.16) (i.e. the transverse positions of the virtual gluon at the emission points) are time-dependent whenever they refer to emissions by the nascent gluon.

Once again, we are mostly interested in the situation where the partonic system created by the fluctuation scatters only once in the medium. This is illustrated in figure 6 and is described by the linearized version of eq. (5.16), as obtained by expanding the exponential. After manipulations similar to those in section 4.3, this can be written as (cf. eq. (4.27))

$$
\begin{aligned}
& \int_{y^{+}}^{x^{+}} \mathrm{d} t \frac{\partial \Gamma_{\omega}(\boldsymbol{u}(t))}{\partial \omega}=-\frac{\alpha_{s} N_{c}}{2} \frac{1}{\omega^{3}} \int_{y^{+}}^{x^{+}} \mathrm{d} t \int \mathrm{d}^{2} \boldsymbol{z}\left[\Gamma_{\omega}(\boldsymbol{u}(t)-\boldsymbol{z})+\Gamma_{\omega}(\boldsymbol{z})-\Gamma_{\omega}(\boldsymbol{u}(t))\right] \\
& \quad \times\left.\left.\int_{y^{+}}^{t} \mathrm{~d} t_{1} \int_{t}^{x^{+}} \mathrm{d} t_{2} \partial_{\boldsymbol{r}_{1}}^{i} \partial_{\boldsymbol{r}_{2}}^{i}\left\{\mathcal{G}_{0}\left(t_{2}-t, \boldsymbol{r}_{2}-\boldsymbol{z} ; \omega\right) \mathcal{G}_{0}\left(t-t_{1}, \boldsymbol{z}-\boldsymbol{r}_{1} ; \omega\right)\right\}\right|_{\boldsymbol{r}_{2}=0} ^{\boldsymbol{r}_{2}=\boldsymbol{u}\left(t_{2}\right)}\right|_{\boldsymbol{r}_{1}=0} ^{\boldsymbol{r}_{1}=\boldsymbol{u}\left(t_{1}\right)},
\end{aligned}
$$

where we recall that $\boldsymbol{z}$ denotes the position of the virtual gluon at the interaction time $t$, with $t_{1}<t<t_{2}$. For the corresponding equation in section 4.3 , we have been able to explicitly perform the integrals over $t_{1}$ and $t_{2}$. Here, however, these integrals are complicated by the time dependence of the endpoints $\boldsymbol{r}_{1}$ and $\boldsymbol{r}_{2}$. To overcome this difficulty, we shall exploit the separation of time scales between the radiated gluon with energy $k^{+}$and the virtual one with energy $\omega \ll k^{+}$. During the lifetime $\Delta t \equiv t_{2}-t_{1} \lesssim \tau_{\mathrm{br}}(\omega)$ of the fluctuation, the transverse position of the hard gluon changes by an amount

$$
\Delta u_{\perp}^{2} \sim \frac{2 \Delta t}{k^{+}} \lesssim \frac{2 \tau_{\mathrm{br}}(\omega)}{k^{+}}
$$

which is small compared to the typical separation $\left\langle u_{\perp}^{2}>\simeq 4 / k_{\mathrm{br}}^{2}\left(k^{+}\right)\right.$between the hard gluon and the quark:

$$
\frac{\Delta u_{\perp}^{2}}{<u_{\perp}^{2}>} \sim \frac{\tau_{\mathrm{br}}(\omega)}{\tau_{\mathrm{br}}\left(k^{+}\right)} \sim \sqrt{\frac{\omega}{k^{+}}} \ll 1 .
$$

Hence, when evaluating the endpoints $\boldsymbol{r}_{1}$ and $\boldsymbol{r}_{2}$ in eq. (5.17), one can neglect the small difference between $\boldsymbol{u}\left(t_{2}\right)$ and $\boldsymbol{u}\left(t_{1}\right)$ and approximate them both with the intermediate value $\boldsymbol{u}(t)$ (the transverse size of the parent dipole at the interaction time). Then the integrals over $t_{1}$ and $t_{2}$ can be done as in eq. (4.28), and one is led to

$$
\int_{y^{+}}^{x^{+}} \mathrm{d} t \omega \frac{\partial \Gamma_{\omega}(\boldsymbol{u}(t))}{\partial \omega}=\frac{\bar{\alpha}}{2 \pi} \int_{y^{+}}^{x^{+}} \mathrm{d} t \int_{\boldsymbol{z}} \frac{\boldsymbol{u}^{2}(t)}{(\boldsymbol{z}-\boldsymbol{u}(t))^{2} \boldsymbol{z}^{2}}\left[\Gamma_{\omega}(\boldsymbol{u}(t)-\boldsymbol{z})+\Gamma_{\omega}(\boldsymbol{z})-\Gamma_{\omega}(\boldsymbol{u}(t))\right] .
$$

This holds for generic values of the integration limits $y^{+}$and $x^{+}$(recall that these variables are themselves integrated over in eq. (5.2)), hence it must hold locally in $t$ :

$$
\omega \frac{\partial \Gamma_{\omega}(\boldsymbol{u}(t))}{\partial \omega}=\frac{\bar{\alpha}}{2 \pi} \int_{\boldsymbol{z}} \frac{\boldsymbol{u}^{2}(t)}{(\boldsymbol{z}-\boldsymbol{u}(t))^{2} \boldsymbol{z}^{2}}\left[\Gamma_{\omega}(\boldsymbol{u}(t)-\boldsymbol{z})+\Gamma_{\omega}(\boldsymbol{z})-\Gamma_{\omega}(\boldsymbol{u}(t))\right] .
$$




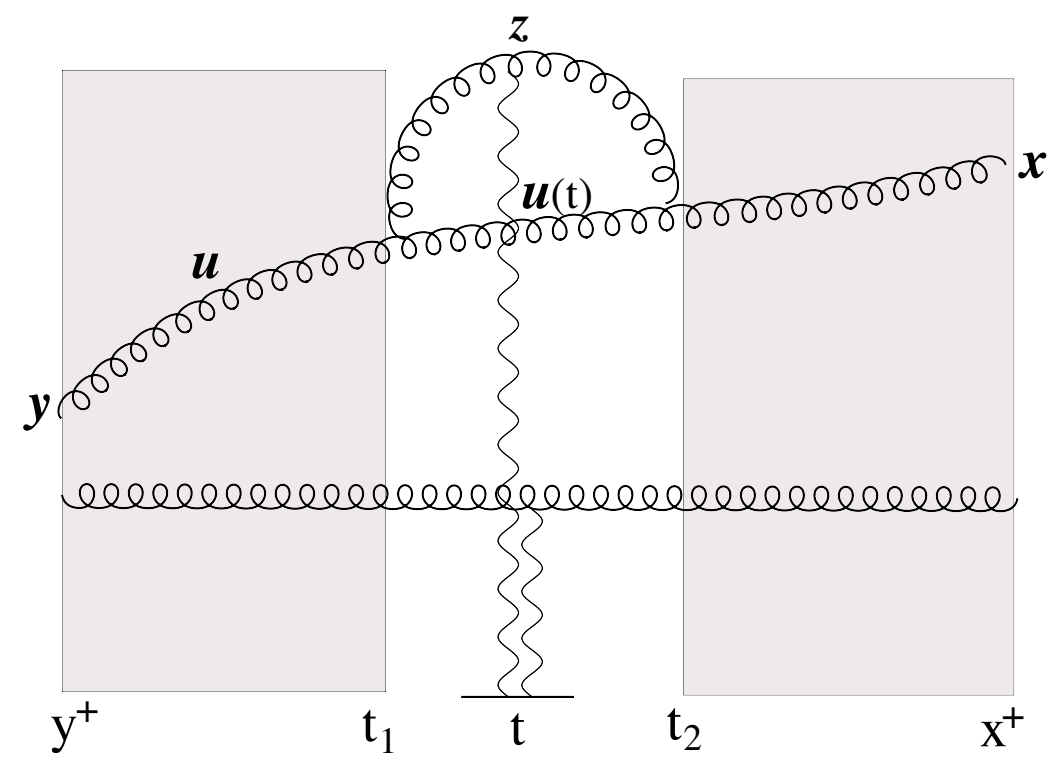

Figure 6. A diagram contributing to the evolution of a non-eikonal dipole, as described by eq. (5.17). The evolving dipole lives fully inside the medium $\left(0<y^{+}<x^{+}<L\right)$. The grey areas prior and after the fluctuation are regions of multiple scattering. During the lifetime of the fluctuation, between $t_{1}$ and $t_{2}$, the partonic system (effectively made with three gluons) scatters only once, at some intermediate time $t$.

This is recognized as the BFKL equation for a dipole with time-dependent transverse size $\boldsymbol{u}(t)$. As clear from its above derivation, this equation is valid so long as the relative change in $\boldsymbol{u}(t)$ remains negligible during the lifetime of the typical fluctuations.

In particular, the time-dependence of $\boldsymbol{u}(t)$ is irrelevant for the double-logarithmic approximation that we are primarily interested in. As explained in section 4.3.2, this is controlled by fluctuations with relatively large transverse sizes, which are only logarithmically sensitive to the parent dipole size. At DLA, eq. (5.21) reduces to an equation like eq. (4.43) which describes the evolution of the jet quenching parameter $\hat{q}_{\mathrm{g}}\left(\tau, p_{\perp}^{2}\right)$ with the longitudinal $(\tau)$ and transverse $\left(p_{\perp}^{2}\right)$ resolution scales. For the problem at hand, the relevant scales are $\tau=\tau_{\mathrm{br}}\left(k^{+}\right)$(the formation time for the radiated gluon) and $p_{\perp}^{2}=k_{\mathrm{br}}^{2}\left(k^{+}\right)=\hat{q}_{\mathrm{g}} \tau_{\mathrm{br}}\left(k^{+}\right)$ (the transverse momentum squared acquired by this gluon during formation). Hence, the leading-order radiative correction reads (compare to eq. (4.41))

$$
\delta \hat{q}_{\mathrm{g}}^{(1)}=\hat{q}_{\mathrm{g}}^{(0)} \frac{\bar{\alpha}}{2} \ln ^{2} \frac{\tau_{\mathrm{br}}\left(k^{+}\right)}{\lambda}=\hat{q}_{\mathrm{g}}^{(0)} \frac{\bar{\alpha}}{8} \ln ^{2} \frac{k^{+}}{\omega_{0}},
$$

with $\omega_{0} \equiv \hat{q}_{\mathrm{g}} \lambda^{2} / 2$. For the energy-loss problem, $k^{+} \sim \omega_{c}$ and $\tau_{\mathrm{br}}\left(k^{+}\right) \sim L$, hence we return to the original version of the logarithm, as appearing in eqs. (4.41) or (4.46).

To summarize, the dominant effect of the radiative corrections on the calculation of medium-induced gluon radiation consists in the renormalization of the jet quenching parameter within the corresponding tree-level calculation. In particular, to DLA accuracy, the energy loss by an energetic quark can be estimated as

$$
\Delta E(L)=\kappa \frac{\alpha_{s} C_{F}}{\pi} \hat{q}_{\mathrm{g}}\left(L, Q_{s}^{2}\right) L^{2} .
$$


By comparing this to eq. (5.11), we conclude that the radiative corrections have the effect to increase the value of the energy loss (via the corresponding increase in $\hat{q}_{\mathrm{g}}$ ) and also to enhance its dependences upon the medium size $L$ and its temperature $T=1 / \lambda$. In particular, if the medium is sufficiently large, one may approach the asymptotic scaling $\Delta E(L) \propto L^{2+\gamma_{s}}$ with $\gamma_{s}=2 \sqrt{\bar{\alpha}}$ the 'saturation exponent' introduced in eq. (4.63). A similar observation has been made in $[15,16]$.

\section{Conclusions and perspectives}

In this paper we have developed the theory for the non-linear evolution of jet quenching and related phenomena to leading order in perturbative QCD at high energy. This theory can be viewed as a generalization of the BK-JIMWLK evolution for 'dilute-dense' scattering to the case of a target with an arbitrary longitudinal extent. This generalization is complicated by the need to go beyond the eikonal approximation in the treatment of multiple scattering and also to explicitly take into account the non-locality of the quantum fluctuations in time. Accordingly, the general evolution equations, such as the generalized BK equation (4.22), are extremely complicated and the construction of exact solutions appears to be prohibitive, except perhaps via numerical methods.

Fortunately, this theory allows for a drastic simplification in so far as the dominant radiative corrections are concerned: as originally noticed in ref. [9], these corrections are enhanced by the double logarithm $\ln ^{2}(L / \lambda)$. This ultimately originates in the familiar, soft and collinear, divergences of bremsstrahlung in QCD. What is non-trivial though, and specific to the problem at hand, is the way how these divergences are cut off by the nonlinear physics of multiple scattering in the medium. These double-logarithmic corrections can be resumed to all orders by solving the relatively simple, linear, equation (4.42), where the non-linear effects enter only via the restriction on the transverse phase-space for single scattering. This equation, which here emerges via controlled approximations from the generalized BK equation alluded to above, has also been inferred from a directly calculation of the relevant Feynman graphs to DLA accuracy [9, 16].

One of our main results here is to explain the emergence of this remarkable simplification, which is the DLA, from a physical perspective. As we discuss in section 4.4, this is a consequence of the special way how gluon saturation occurs in a medium: the saturation momentum $Q_{s}^{2}(x)$ is proportional to the longitudinal wavelength of the gluons and hence it increases very fast with $1 / x$ already in the absence of the quantum evolution. This in turn implies that the transverse phase-space grows as fast as the longitudinal one when increasing the medium size $L$, thus favoring a double-logarithmic evolution.

Within this double-logarithmic approximation, the radiative corrections are sufficiently mild to be absorbed into a renormalization of the jet quenching parameter, which then evolves according to eq. (4.42). Here, we have demonstrated this property for two particular observables, the transverse momentum broadening and the radiative energy loss, which in the approximations of interest are both related to the $S$-matrix of a color dipole. It would be interesting to understand whether a similar property remains true in more general situations and for more complicated observables, which are sensitive to other correlations 
of the Wilson lines, like the quadrupole. Examples in that sense include the calculation of the medium-induced gluon radiation beyond the eikonal approximation (for the parent parton) $[49,51,56]$, the study of color (de)coherence for multi-gluon emissions inside a medium [77, 79-81], and the evolution of a jet via successive medium-induced parton branchings [71, 78, 82]. A more general calculation, involving a 3-point function of the Wilson lines in the context of medium-induced gluon branching, has been presented in [16].

It would be furthermore interesting to have a deeper understanding of the systematics of the single-logarithmic corrections, i.e. the terms of order $\bar{\alpha} \ln (L / \lambda)$, or $\bar{\alpha} \ln (1 / x)$, in the evolution equations. As discussed in section 4.5, the situation is quite different in that respect from the BK-JIMWLK evolution: the individual terms in the multiple scattering series are not separately enhanced by a large logarithm $\ln (1 / x)$, yet they contribute to leading-logarithmic accuracy in a non-perturbative way - their resummation limits the phase-space for the single scattering approximation. It is not clear to us whether, in this context, it is possible or even useful to explicitly isolate the terms enhanced by $\ln (1 / x)$ within the evolution equations.

An obviously important, open, problem refers to the inclusion of perturbative corrections of higher loop order, such as the running of the QCD coupling. As noticed in section 4.4, the leading order correction to the saturation exponent is quite large for realistic values of $\alpha_{s}$, a situation which generally signals the importance of higher-order corrections. A similar problem occurs for the saturation exponent of a shockwave and in that case we know that the resummation of higher-order effects drastically reduces the leading-order estimate (roughly by a factor of 3 ) $[83,84]$. The calculation of the NLO corrections to the BK-JIMWLK equations has just been completed [85, 86], but the corresponding program for the physics of jet quenching is still awaiting.

Also, it would be important to develop numerical techniques for attacking functional evolution equations like the generalized BK equation (4.22). The original BK equation turned out to be a formidable tool for the phenomenology of particle production in $p p$, $p A$, and even $A A$ collisions (especially after being supplemented with running coupling effects) $[36,87]$, and it would be very useful to dispose of a similar tool for the phenomenology of jet quenching.

Last but not least, it is interesting to notice the convergence between some of the present results at weak coupling, e.g. the saturation exponent for the plasma, or the $L$ dependence of the renormalized $\hat{q}$ and of the energy loss, and the corresponding results at strong coupling ${ }^{21}[72-74,90-92]$. One may view this as merely a coincidence, but we do not believe so: as discussed in refs. [73, 74], the dominant mechanism for transverse momentum broadening in a strongly coupled plasma is the recoil associated with mediuminduced radiation. (That is, at strong coupling, the same mechanism is responsible for both energy loss and momentum broadening.) The perturbative corrections that we have considered here at weak coupling are themselves associated with radiation, so their inclusion naturally interpolates towards the physical scenario expected at strong coupling.

\footnotetext{
${ }^{21}$ More precisely, we mean here the results concerning the energy loss and momentum broadening of light partons, which is the most interesting case in the high-energy limit. For a more general survey of the related AdS/CFT literature, including the important case of a heavy quark, we refer to the review papers [88, 89].
} 
And indeed, the present perturbative results, which as we have seen predict a (global) saturation momentum $Q_{s}^{2}(L)=\hat{q}(L) L \propto L^{1+\gamma_{s}}$ and an energy loss $\Delta E(L) \propto L^{2+\gamma_{s}}$ with $\gamma_{s}=2 \sqrt{\bar{\alpha}}$, suggest a relatively smooth approach towards the respective trends at strong coupling, namely $Q_{s}^{2}(L) \propto L^{2}[72,74]$ and respectively $\Delta E(L) \propto L^{3}[73,74,90-92]$. It remains to be seen whether such a smooth convergence survives after including higher order perturbative corrections.

\section{Acknowledgments}

I am grateful to Al Mueller for many insightful discussions, for repeatedly reading the manuscript, and for helping me clarifying some of the arguments. During the gestation of this work, I have benefited from many inspiring discussions with my colleagues in Saclay, Jean-Paul Blaizot, Fabio Dominguez, Yacine Mehtar-Tani, and Bin Wu. This research is supported by the European Research Council under the Advanced Investigator Grant ERC-AD-267258. All figures were made with Jaxodraw [93].

\section{A A succinct derivation of the evolution Hamiltonian}

In this appendix, we shall provide a succinct proof of the evolution Hamiltonian (2.4), by generalizing the original construction of the JIMWLK Hamiltonian [21-29] to the case where the target is a extended medium, as opposed to a shockwave. Our subsequent discussion is rather schematic and we refer to the original literature on JIMWLK for more details.

A central ingredient in this construction is a factorization scheme for the $S$-matrix describing the high-energy scattering between a dilute projectile and a dense target. This scheme is most naturally formulated in a 'target infinite momentum frame', i.e. a Lorentz frame where the target propagates at nearly the speed of light, so that the typical time scales for its internal dynamics are Lorentz dilated. In the same frame, the interaction with an external projectile appears as a relatively fast process, whose duration is set by the Lorentz-contracted size of the projectile. Hence, for the purposes of computing that scattering, one can describe the target as a 'frozen' configuration of color fields, which is random - since determined by the instantaneous distribution of fast moving 'color sources' (the medium constituents) - and must be averaged over in the calculation of the observables. This discussion motivates the 'color glass condensate factorization', which has originally been formulated for the case of a shockwave target [35, 36, 67, 68], but on the basis of very general ideas, which apply to an extended medium as well.

To describe this factorization, we shall assume that the target is a left mover (hence the projectile is a right mover), and that the rapidity separation between the valence degrees of freedom of the two hadronic systems is equal to $Y$. We work in a Lorentz frame where $Y=Y_{T}+Y_{P}$, with the positive quantities $Y_{T}$ and $Y_{P}$ denoting the magnitudes of the rapidities for the target and the projectile, respectively. The partonic constituents of the projectile couple to the component $A^{-}$of the target color field via Wilson lines, as shown in eq. (2.3). The overall $S$-matrix is then represented by some color-singlet operator $\hat{\mathcal{O}}_{Y_{P}}\left[A^{-}\right]$, built with these Wilson lines. This is an 'operator' since defined for a given configuration of 
the color fields in the target. But the physical observable is of course the average $S$-matrix, as obtained after averaging this operator over all the realizations of the random field $A^{-}$, with some suitable (functional) probability density, known as the 'CGC weight function' $W_{Y_{T}}\left[A^{-}\right]$:

$$
\langle\hat{\mathcal{O}}\rangle_{Y}=\int\left[D A^{-}\right] W_{Y_{T}}\left[A^{-}\right] \hat{\mathcal{O}}_{Y_{P}}\left[A^{-}\right]
$$

As indicated by the notations above, the average $S$-matrix depends only upon the total rapidity separation $Y$, by boost invariance. The CGC weight function $W_{Y_{T}}\left[A^{-}\right]$encodes the relevant information about the target wavefunction (including its high-energy evolution up to rapidity $\left.Y_{T}\right)$ in the approximations of interest.

So far, the CGC factorization in eq. (A.1) is merely an assumption. At 'tree-level' (i.e. in the zeroth order approximation of pQCD, valid so long as $\bar{\alpha} Y \ll 1$ ), this is motivated by the separation of scales between the valence degrees of freedom in the target and the projectile, as discussed. But in order to justify this factorization for a larger rapidity difference $Y \gtrsim 1 / \bar{\alpha}$, one needs to demonstrate that it is preserved by the quantum corrections responsible for the high-energy evolution. In what follows, we shall construct a proof in that sense via induction. Namely, assuming that eq. (A.1) holds after a generic number of steps in the evolution, we shall explicitly perform one additional such a step - by integrating out one layer of (relatively soft) quantum fluctuations within pQCD - and show that the result of that calculation can be rewritten as in eq. (A.1), but with a modified expression for the $S$-matrix operator ('projectile evolution'), or, alternatively, for the CGC weight function ('target evolution'). The respective change in either the $S$-matrix, or the CGC weight function, can be used to deduce the evolution Hamiltonian and thus check eq. (2.4).

In turns out that it is easier to perform one step in the high-energy evolution by boosting the projectile, rather than the target. Indeed, since the projectile is dilute, the evolution of its wavefunction remains linear (i.e. of the BFKL type). Then the only nonlinear effects that one needs to take into account are those associated with the multiple scattering off the target field, as encoded in the Wilson lines. By boost invariance, one is guaranteed that the same evolution Hamiltonian would also be obtained by boosting the target, albeit the respective calculation would be more involved, because of the need to deal with gluon saturation in the dense target wavefunction. (For the case of a shockwave target, both the projectile and the target evolutions have been explicitly worked and shown to be equivalent with each other [19-29]. The viewpoint of projectile evolution naturally leads to the Balitsky hierarchy of coupled equations for $S$-matrices, while that of target evolution yields the functional JIMWLK equation for the CGC weight function.)

So, let us increase the rapidity of the projectile according to $Y_{P} \rightarrow Y_{P}+\Delta Y$. To understand the consequences of this boost, let us first remind some general facts about the high-energy evolution:

(i) The wavefunction of the projectile with rapidity $Y_{P}$ includes quanta - the valence partons and the relatively soft gluons produced via radiation - with longitudinal momenta $p^{+}$within the strip $\Lambda_{0}<p^{+}<\Lambda_{0} \mathrm{e}^{Y_{P}}$. Here, $\Lambda_{0}$ is the infrared cutoff used 
to properly define the wavefunction (the softest longitudinal momentum that can be measured).

(ii) When the projectile is boosted by an amount $\Delta Y$, the already existing partons act as sources for the emission of additional gluons within the range $\Lambda_{0}<p^{+}<\Lambda_{0} \mathrm{e}^{\Delta Y}$. The new gluons are much softer than their sources (whose typical $p^{+}$momenta are very large as compared to $\Lambda_{0} \mathrm{e}^{\Delta Y}$ ), so their emission can be computed in the 'soft gluon' approximation, i.e. by using eikonal vertices.

(iii) Albeit soft relative to their sources, the 'evolution' gluons are still fast enough as compared to the target, so their scattering off the latter can be described by Wilson lines.

(iv) The probability for a soft gluon emission within the range $\Lambda_{0}<p^{+}<\Lambda_{0} \mathrm{e}^{\Delta Y}$ is of or$\operatorname{der} \bar{\alpha} \Delta Y$, with $\bar{\alpha} \equiv \alpha_{s} N_{c} / \pi$. Hence, by keeping $\Delta Y \ll 1 / \bar{\alpha}$, one can ensure that there is only one additional gluon emission, which can be treated in perturbation theory.

These general considerations hold irrespectively of the nature of the dense target a shockwave or an extended medium. However, the respective calculations differ in one important aspect: the emission of a soft gluon in the background of a shockwave can be treated in the eikonal approximation, that is, one can neglect the transverse diffusion of the gluon while crossing the shockwave; but for an extended medium the eikonal approximation fails, since gluon fluctuations can scatter during their whole lifetime.

We are now prepared to describe the calculation of one step in the high-energy evolution. To that aim, we need a generalization of eq. (A.1) which allows for the relevant quantum fluctuations. This is obtained by inserting into eq. (A.1) the QCD path integral which describes the dynamics of the quantum gluons with longitudinal momenta within the range $\Lambda_{0}<\left|p^{+}\right|<\Lambda_{0} \mathrm{e}^{\Delta Y}$, in the high-energy approximations of interest. The appropriate generalization of eq. (A.1) reads

$$
\langle\hat{\mathcal{O}}\rangle_{Y+\Delta Y}=\int\left[D A^{-}\right] W_{Y_{T}}\left[A^{-}\right] Z_{\Delta Y}^{-1} \int_{\Delta Y}\left[D a^{\mu}\right] \delta\left(a^{+}\right) \mathrm{e}^{\mathrm{i} S_{0}\left[a^{\mu} ; A^{-}\right]} \hat{\mathcal{O}}_{Y_{P}}\left[A^{-}+a^{-}\right] .
$$

Here $a^{\mu}(x)$ are the gauge fields representing the quantum fluctuations and we use the projectile light-cone gauge $a^{+}=0$. The normalization factor $Z_{\Delta Y}$ is given by a similar path integral, but without the factor $\hat{\mathcal{O}}_{Y_{P}}$.

The 'soft' gluons $a^{\mu}$ couple to their 'sources' (the projectile partons at higher rapidities that have been included in the $S$-matrix $\hat{\mathcal{O}}_{Y_{P}}$ ) and to the target color field $A^{\mu} \simeq \delta^{\mu-} A^{-}$ (the 'background field'). The coupling to the 'sources' is implemented via the shift $A^{-} \rightarrow$ $A^{-}+a^{-}$in the functional argument of the scattering operator $\hat{\mathcal{O}}_{Y_{P}}$. This is correct since the fast partons interact in the same way — via Wilson lines - with both the target and their (comparatively soft) quantum fluctuations. The coupling to $A^{-}$is encoded in the action $S_{0}\left[a^{\mu} ; A^{-}\right]$, which is obtained by keeping only the terms quadratic in $a^{\mu}$ in the expansion of the Yang-Mills action $S_{Y M}\left[A^{\mu}+a^{\mu}\right]$ around the background field:

$$
S_{0}\left[a^{\mu} ; A^{-}\right]=\frac{1}{2} \int \mathrm{d}^{4} x\left[a^{i}\left(-D^{2}\right) a^{i}+\left(\partial^{+} a^{-}+\partial_{i} a^{i}\right)^{2}\right],
$$


where $D^{2}=2 \partial^{+} D^{-}-\nabla_{\perp}^{2}$, with $D^{-}=\partial^{-}-i g A^{-}$(the covariant derivative built with the background field) and $\partial_{i}=\partial / \partial x^{i}=-\partial^{i}$. The action (A.3) is formally written as a 4-dimensional integral, but the integrand is homogeneous in $x^{-}$and it is understood that the integral over the corresponding modes $p^{+}$is restricted to the strip $\Lambda_{0}<\left|p^{+}\right|<\Lambda_{0} \mathrm{e}^{\Delta Y}$. The quadratic action (A.3) generates, as usual, the propagator $G^{\mu \nu}$ of the soft gluons in the background field and in the LC gauge $a^{+}=0$. Namely, eq. (A.3) is tantamount to

$$
i S_{0}\left[a^{\mu} ; A^{-}\right]=-\frac{1}{2} \int_{\text {strip }} \frac{\mathrm{d} p^{+}}{2 \pi} \int_{x^{+}, y^{+}} \int_{\boldsymbol{x}, \boldsymbol{y}} a_{b}^{\mu}\left(x^{+}, \boldsymbol{x}, p^{+}\right) G_{\mu \nu}^{-1, b c}\left(x^{+}, \boldsymbol{x} ; y^{+}, \boldsymbol{y} ; p^{+}\right) a_{c}^{\nu}\left(y^{+}, \boldsymbol{y}, p^{+}\right),
$$

where $G^{\mu \nu}$ is the background-field gluon propagator in the LC gauge, to be constructed in appendix B. This propagator encodes both the transverse diffusion and the multiple scattering off the target field.

In writing the action (A.3) we have ignored the self-interactions of the quantum gluons, which is indeed justified to leading order in $\alpha_{s}$. (As a general rule, the target field is strong, $g A^{-} \sim \mathcal{O}(1)$, and must be treated exactly, whereas the quantum fields are weak, $g a^{\mu} \ll 1$, and should be expanded out in perturbation theory.) For consistency, one must also expand the scattering operator $\hat{\mathcal{O}}_{Y_{P}}\left[A^{-}+a^{-}\right]$in powers of $a^{-}$, up to quadratic order. This is tantamount to considering a single gluon emission, as expected for one step in the high-energy evolution. The linear term in this expansion vanishes after computing the path integral over $a^{\mu}$, whereas the quadratic term yields a contribution proportional to $G^{--}$. In fact, this quadratic term can be recognized as the action of the Hamiltonian $\Delta H$ introduced in eq. (2.4) on the original operator $\hat{\mathcal{O}}_{Y_{P}}\left[A^{-}\right]$. After this expansion, eq. (A.2) reduces to

$$
\langle\hat{\mathcal{O}}\rangle_{Y+\Delta Y}-\langle\hat{\mathcal{O}}\rangle_{Y}=\int\left[D A^{-}\right] W_{Y_{T}}\left[A^{-}\right] \Delta H \hat{\mathcal{O}}_{Y_{P}}\left[A^{-}\right]
$$

with $\Delta H$ given by eq. (2.4) with $\Lambda \equiv \Lambda_{0} \mathrm{e}^{\Delta Y}$ and $x \equiv \mathrm{e}^{-\Delta Y}$ (and therefore $x \Lambda=\Lambda_{0}$ ).

Eq. (A.5) represents the result of one step in the high-energy evolution to the accuracy of interest. This equation can be interpreted as an evolution equation for the $S$-matrix operator:

$$
\hat{\mathcal{O}}_{Y_{P}+\Delta Y}\left[A^{-}\right]-\hat{\mathcal{O}}_{Y_{P}}\left[A^{-}\right]=\Delta H \hat{\mathcal{O}}_{Y_{P}}\left[A^{-}\right] .
$$

From this perspective, $\Delta H$ describes the evolution of the projectile wavefunction as measured by the multiple scattering off the dense target. This is the sense in which we have generally used $\Delta H$ as an 'evolution Hamiltonian' throughout this paper. Eq. (A.6) can be viewed as the generalization of the Balitsky equations to the case of an extended target.

Alternatively, within the functional integral in eq. (A.5), one can integrate by parts the functional derivatives implicit in the structure of $\Delta H$ (cf. eq. (2.4)) and thus make them act on the CGC weight function $W_{Y_{T}}\left[A^{-}\right]$. Accordingly, the effect of the evolution can be reinterpreted as a renormalization of the CGC target wavefunction (a generalization of the JIMWLK equation):

$$
W_{Y_{T}+\Delta Y}\left[A^{-}\right]-W_{Y_{T}}\left[A^{-}\right]=\Delta H W_{Y_{T}}\left[A^{-}\right] .
$$


To summarize, the effect of integrating out quantum gluons in one layer of rapidity amounts to replacing the starting point formula (A.1) by either

$$
\langle\hat{\mathcal{O}}\rangle_{Y+\Delta Y}=\int\left[D A^{-}\right] W_{Y_{T}}\left[A^{-}\right] \hat{\mathcal{O}}_{Y_{P}+\Delta Y}\left[A^{-}\right]
$$

or

$$
\langle\hat{\mathcal{O}}\rangle_{Y+\Delta Y}=\int\left[D A^{-}\right] W_{Y_{T}+\Delta Y}\left[A^{-}\right] \hat{\mathcal{O}}_{Y_{P}}\left[A^{-}\right]
$$

with $\hat{\mathcal{O}}_{Y_{P}+\Delta Y}\left[A^{-}\right]$and $W_{Y_{T}+\Delta Y}\left[A^{-}\right]$defined in eqs. (A.6) and (A.7), respectively. Any of these expressions shows that the CGC factorization is preserved by one step in the high-energy evolution, which closes our inductive argument.

\section{B The background field gluon propagator}

In this appendix, we construct the background field gluon propagator in the light-cone gauge $A^{+}=0$ and collect some related formulæ that were used in the main text. Our presentation will be brief since similar constructions can be found in the literature. (See e.g. section 6 in ref. [26] for a related discussion.) The propagator is defined as in appendix A, that is,

$$
\begin{aligned}
G_{a b}^{\mu \nu}(x, y) & \equiv\left\langle\mathrm{T} a_{a}^{\mu}(x) a_{b}^{\nu}(y)\right\rangle \\
& =Z^{-1} \int\left[D a^{\mu}\right] \delta\left(a^{+}\right) a_{a}^{\mu}(x) a_{b}^{\nu}(y) \mathrm{e}^{\mathrm{i} S_{0}\left[a^{\mu} ; A^{-}\right]}
\end{aligned}
$$

where the symbol $\mathrm{T}$ refers to operator ordering in LC time $\left(x^{+}\right)$and $x^{\mu}=\left(x^{+}, x^{-}, \boldsymbol{x}\right)$. It is implicitly assumed that the longitudinal momentum $p^{+}$of the quantum fluctuations is restricted to the strip (2.5), whereas the background field $A^{\mu}=\delta^{\mu-} A^{-}$carries no $p^{+}$ momentum (i.e. it is homogeneous in $x^{-}$). The action $S_{0}\left[a^{\mu} ; A^{-}\right]$is shown in eq. (A.3) and is quadratic in the quantum fields $a^{\mu}$. It is convenient to bring this action to a diagonal form, by replacing $a^{-} \rightarrow \tilde{a}^{-}$with

$$
\tilde{a}^{-}(x) \equiv a^{-}(x)+\frac{\partial^{i}}{\partial^{+}} a^{i}(x) .
$$

Then the action becomes (we recall that $D^{2}=2 \partial^{+} D^{-}-\nabla_{\perp}^{2}$ and $D^{-}=\partial^{-}-i g A^{-}$)

$$
S_{0}\left[\tilde{a}^{-}, a^{i} ; A^{-}\right]=\frac{1}{2} \int \mathrm{d}^{4} x\left[a^{i}\left(-D^{2}\right) a^{i}+\left(\partial^{+} \tilde{a}^{-}\right)^{2}\right],
$$

which implies that the propagator $G^{i j}$ of the transverse fields is the same as the 'scalar' propagator: $G^{i j}=\delta^{i j} G$, with $G_{a b}(x, y)$ obeying eq. (2.9) (after a Fourier transform $x^{-}-$ $\left.y^{-} \rightarrow p^{+}\right)$. Also,

$$
\left\langle\mathrm{T} \tilde{a}_{a}^{-}(x) \tilde{a}_{b}^{-}(y)\right\rangle=\delta_{a b} \int \frac{\mathrm{d}^{4} p}{(2 \pi)^{4}} \mathrm{e}^{-\mathrm{i} p \cdot(x-y)} \frac{\mathrm{i}}{\left(p^{+}\right)^{2}}, \quad\left\langle\mathrm{~T} \tilde{a}_{a}^{-}(x) a_{b}^{i}(y)\right\rangle=0 .
$$


After inverting the transformation in eq. (B.2), we finally obtain [in the mixed Fourier representation $\left(\vec{x}, p^{+}\right)$with $\vec{x} \equiv\left(x^{+}, \boldsymbol{x}\right)$ and color indices suppressed]

$$
\begin{aligned}
G^{-i}\left(\vec{x}, \vec{y} ; p^{+}\right) & =\frac{\mathrm{i}}{p^{+}} \partial_{\boldsymbol{x}}^{i} G\left(\vec{x}, \vec{y} ; p^{+}\right), \quad G^{i-}\left(\vec{x}, \vec{y} ; p^{+}\right)=-\frac{\mathrm{i}}{p^{+}} \partial_{\boldsymbol{y}}^{i} G\left(\vec{x}, \vec{y} ; p^{+}\right), \\
G^{--}\left(\vec{x}, \vec{y} ; p^{+}\right) & =\frac{1}{\left(p^{+}\right)^{2}} \partial_{\boldsymbol{x}}^{i} \partial_{\boldsymbol{y}}^{i} G\left(\vec{x}, \vec{y} ; p^{+}\right)+\frac{\mathrm{i}}{\left(p^{+}\right)^{2}} \delta^{(3)}(\vec{x}-\vec{y}) .
\end{aligned}
$$

The propagator is to be considered with the Feynman prescription for the pole at the mass-shell. For instance, the free $\left(A^{-}=0\right)$ scalar propagator reads $G_{0, a b}=\delta_{a b} G_{0}$, with

$$
G_{0}(p)=\frac{\mathrm{i}}{2 p^{+} p^{-}-\boldsymbol{p}^{2}+i \epsilon},
$$

in momentum space. This implies e.g.

$$
G_{0}^{--}(p)=\frac{\boldsymbol{p}^{2}}{\left(p^{+}\right)^{2}} G_{0}(p)+\frac{\mathrm{i}}{\left(p^{+}\right)^{2}}=\frac{2 p^{-}}{p^{+}} \frac{\mathrm{i}}{2 p^{+} p^{-}-\boldsymbol{p}^{2}+i \epsilon},
$$

The 'axial' pole at $p^{+}=0$ needs no special prescription, since $p^{+}$cannot vanish within the present context, as manifest on eq. (2.5). The expression of the free propagator in mixed Fourier representation will also be useful:

$$
G_{0}\left(t, \boldsymbol{x} ; p^{+}\right)=\frac{1}{2 p^{+}}\left[\theta(t) \theta\left(p^{+}\right)-\theta(-t) \theta\left(-p^{+}\right)\right] \int \frac{\mathrm{d}^{2} \boldsymbol{p}}{(2 \pi)^{2}} \mathrm{e}^{\mathrm{i} \boldsymbol{p} \cdot \boldsymbol{x}} \mathrm{e}^{-\mathrm{i} \frac{p_{\perp}^{2}}{2 p^{+}} t} .
$$

Note that modes with positive (negative) values of $p^{+}$propagate forward (backward) in time.

A formal expression for the 'scalar' propagator, which is valid for an arbitrary background field but involves a path integral, has been presented in eq. (2.10). Using this formal expression, we shall now to derive a fully explicit formula for the case where the target is a shockwave localized near $x^{+}=0$. When $x^{+}$and $y^{+}$are both positive, or both negative (i.e. they are on the same side of the shockwave), then the propagator in eq. (2.10) reduces to the free propagator $G_{0}$. Consider now the case where the gluon crosses the shockwave: $y^{+}<0$ and $x^{+}>0$. Then for a localized target field $A^{-}(t, \boldsymbol{z}) \sim \delta(t)$, one can approximate the Wilson line in eq. (2.10) as

$$
U_{x^{+} y^{+}}^{\dagger}[\boldsymbol{r}(t)] \simeq \mathrm{Pe}^{\mathrm{i} g \int \mathrm{d} t A^{-}(t, \boldsymbol{r}(0))}=\int \mathrm{d}^{2} \boldsymbol{z} \delta^{(2)}(\boldsymbol{z}-\boldsymbol{r}(0)) \mathrm{P} \mathrm{e}^{\mathrm{i} g \int \mathrm{d} t A^{-}(t, \boldsymbol{z})},
$$

and the path integral can be computed as follows:

$$
\int[\mathcal{D} \boldsymbol{r}(t)] \exp \left\{\mathrm{i} \frac{p^{+}}{2} \int_{y^{+}}^{x^{+}} \mathrm{d} t \dot{\boldsymbol{r}}^{2}(t)\right\} \delta^{(2)}(\boldsymbol{z}-\boldsymbol{r}(0))=\mathcal{G}_{0}\left(x^{+}, \boldsymbol{x}-\boldsymbol{z} ; p^{+}\right) \mathcal{G}_{0}\left(-y^{+}, \boldsymbol{z}-\boldsymbol{y} ; p^{+}\right),
$$

where $\mathcal{G}_{0}=2 p^{+} G_{0}$ is the free 'reduced' propagator. The last remaining case, where $y^{+}>0$ and $x^{+}<0$, can be deduced by using the symmetry property (2.11). One finally has

$$
\begin{aligned}
& G\left(x^{+}, \boldsymbol{x} ; y^{+}, \boldsymbol{y} ; p^{+}\right)= G_{0}\left(x^{+}-y^{+}, \boldsymbol{x}-\boldsymbol{y} ; p^{+}\right)\left[\theta\left(x^{+}\right) \theta\left(y^{+}\right)+\theta\left(-x^{+}\right) \theta\left(-y^{+}\right)\right] \\
&+2 p^{+} \int_{\boldsymbol{z}} G_{0}\left(x^{+}, \boldsymbol{x}-\boldsymbol{z} ; p^{+}\right) G_{0}\left(-y^{+}, \boldsymbol{z}-\boldsymbol{y} ; p^{+}\right) \\
& \times\left[\theta\left(x^{+}\right) \theta\left(-y^{+}\right) U_{\boldsymbol{z}}^{\dagger}-\theta\left(-x^{+}\right) \theta\left(y^{+}\right) U_{\boldsymbol{z}}\right] .
\end{aligned}
$$




\section{A sum rule for the light-cone gauge propagator}

In this appendix, we shall demonstrate the identity (2.13) which has played an important role in the construction of the high-energy evolution equations, notably in relation with the probability conservation and the cancellation of ultraviolet divergences. The careful treatment of the $\epsilon$-dependence introduced by the adiabatic prescription will be essential for this purpose. Specifically, we shall show that the double time integral in the l.h.s. of (2.13) gives a result of $\mathcal{O}(\epsilon)$ and hence vanishes in the limit $\epsilon \rightarrow 0$.

We separately consider the two pieces in the decomposition (B.7) of the free propagator and use the mixed Fourier representation $G_{0}\left(t_{2}-t_{1}, \boldsymbol{p} ; p^{+}\right)$, cf. eq. (B.8). We focus on the case $p^{+}>0$ for definiteness. Then the 'radiation' piece of the propagator is retarded $\left(\propto \theta\left(t_{2}-t_{1}\right)\right)$ and yields

$$
\begin{aligned}
\int \mathrm{d} t_{1} & \int \mathrm{d} t_{2} G_{0, \text { rad }}^{--}\left(t_{2}-t_{1}, \boldsymbol{p} ; p^{+}\right) \mathrm{e}^{-\epsilon\left(\left|t_{1}\right|+\left|t_{2}\right|\right)} \\
& =\frac{p_{\perp}^{2}}{2\left(p^{+}\right)^{3}} \int_{-\infty}^{\infty} \mathrm{d} t_{2} \int_{-\infty}^{t_{2}} \mathrm{~d} t_{1} \mathrm{e}^{-\mathrm{i} p^{-}\left(t_{2}-t_{1}\right)} \mathrm{e}^{-\epsilon\left(\left|t_{1}\right|+\left|t_{2}\right|\right)} \\
& =\frac{p_{\perp}^{2}}{2\left(p^{+}\right)^{3}} \frac{1}{2 \epsilon}\left[\frac{1}{\mathrm{i} p^{-}+\epsilon}+\frac{1}{\mathrm{i} p^{-}-\epsilon}\right]=-\frac{1}{\epsilon} \frac{\mathrm{i}}{\left(p^{+}\right)^{2}}+\mathcal{O}(\epsilon),
\end{aligned}
$$

where $p^{-} \equiv p_{\perp}^{2} / 2 p^{+}$. Note that, as compared to the previous, related, calculation in eq. (3.12), the final result here is a purely divergent contribution, without any additional finite term. The respective contribution of the Coulomb piece is, clearly,

$$
\int \mathrm{d} t_{1} \int \mathrm{d} t_{2} G_{0, \text { Coul }}^{--}\left(t_{2}-t_{1}, \boldsymbol{p} ; p^{+}\right) \mathrm{e}^{-\epsilon\left(\left|t_{1}\right|+\left|t_{2}\right|\right)}=\frac{\mathrm{i}}{\left(p^{+}\right)^{2}} \int_{-\infty}^{\infty} \mathrm{d} t \mathrm{e}^{-2 \epsilon|t|}=\frac{1}{\epsilon} \frac{\mathrm{i}}{\left(p^{+}\right)^{2}} .
$$

As anticipated, this precisely cancels the divergent piece of the 'radiative' contribution, thus leaving a net result of $\mathcal{O}(\epsilon)$.

\section{Finite- $N_{c}$ corrections within the mean field approximation}

When constructing the evolution equation for a dipole in a medium, in section 4.2 , we have used the large- $N_{c}$ limit to simplify some arguments and the various formulæ. But as also announced there, all the results obtained within the Gaussian approximation (4.14) for the medium correlations can be extended to finite values for $N_{c}$. In this appendix, we present some tools which are useful in that sense. Such tools have been developed in applications of the CGC formalism and we refer to the original literature for their derivation and more details [37, 39-41, 43, 44].

As visible e.g. on (4.15), the evolution of the dipole $S$-matrix within the Gaussian approximation involves only two distinct Wilson line correlators: the dipole itself and a correlator built with three Wilson lines for the partonic system which exists during the fluctuation. For more generality, let us consider a dipole in some generic representation $R$ of the $\mathrm{SU}\left(N_{c}\right)$ algebra. Then, the relevant correlators read (within the mean field approximation, of course)

$$
\mathcal{S}_{R}(\boldsymbol{x}, \boldsymbol{y}) \equiv \frac{1}{d_{R}}\left\langle\operatorname{tr}_{R}\left[V_{R}^{\dagger}(\boldsymbol{x}) V_{R}(\boldsymbol{y})\right]\right\rangle=\exp \left\{-g^{2} C_{R} \int \mathrm{d} t \Gamma(t, \boldsymbol{x}, \boldsymbol{y})\right\}
$$


and respectively (see e.g. appendix B in ref. [40] for a rapid derivation)

$$
\left\langle U^{\dagger a b}(\boldsymbol{z}) \frac{\operatorname{tr}_{R}}{d_{R}}\left(t_{R}^{a} V_{R}^{\dagger}(\boldsymbol{x}) t_{R}^{b} V_{R}(\boldsymbol{y})\right)\right\rangle=C_{R} \mathrm{e}^{-g^{2} \int \mathrm{d} t\left[\frac{N_{c}}{2}(\Gamma(t, \boldsymbol{x}, \boldsymbol{z})+\Gamma(t, \boldsymbol{z}, \boldsymbol{y}))-\left(\frac{N_{c}}{2}-C_{R}\right) \Gamma(t, \boldsymbol{x}, \boldsymbol{y})\right]}
$$

In these expressions, $d_{R}$ is the dimension of the representation $\left(d_{F}=N_{c}\right.$ for the fundamental, $d_{A}=N_{c}^{2}-1$ for the adjoint, etc.), $C_{R}$ is the corresponding second Casimir $\left(C_{F}=\left(N_{c}^{2}-1\right) / 2 N_{c}, C_{A}=N_{c}\right.$, etc. $)$, and the integrals over $t$ run over some arbitrary time interval (e.g. the width of the target, or a slice of it). Also, the transverse coordinates $\boldsymbol{x}, \boldsymbol{y}$, and $\boldsymbol{z}$ need not be constant during that time interval, that is, eq. (D.2) also holds for generic trajectories $\boldsymbol{x}(t)$, etc. Finally, the function $\Gamma(t, \boldsymbol{x}, \boldsymbol{y})$ is related to the function $\bar{\Gamma}(t, \boldsymbol{x}, \boldsymbol{y})$ which enters the 2-point function (4.14) via

$$
\Gamma(t, \boldsymbol{x}, \boldsymbol{y})=\frac{1}{2}[\bar{\Gamma}(t, \boldsymbol{x}, \boldsymbol{x})+\bar{\Gamma}(t, \boldsymbol{y}, \boldsymbol{y})]-\bar{\Gamma}(t, \boldsymbol{x}, \boldsymbol{y}) .
$$

Using these formulæ, it is straightforward to generalize the results in section 4.2 to arbitrary $N_{c}$. For instance, for a dipole in the color representation $R$, the analog of eq. (4.22) is obtained by replacing

$$
\begin{aligned}
& \frac{N_{c}}{2}\left[\mathcal{S}_{t_{2}, t_{1}}(\boldsymbol{x}, \boldsymbol{r}) \mathcal{S}_{t_{2}, t_{1}}(\boldsymbol{r}, \boldsymbol{y}) \mathcal{S}_{t_{2}, t_{1}}^{-1}(\boldsymbol{x}, \boldsymbol{y})-1\right] \\
& \rightarrow C_{R}\left\{\mathrm{e}^{-\frac{g^{2} N_{c}}{2} \int_{t_{1}}^{t_{2}} \mathrm{~d} t\left[\Gamma_{\omega}(t, \boldsymbol{x}, \boldsymbol{r})+\Gamma_{\omega}(t, \boldsymbol{r}, \boldsymbol{y})-\Gamma_{\omega}(t, \boldsymbol{x}, \boldsymbol{y})\right]}-1\right\}
\end{aligned}
$$

within the r.h.s. of eq. (4.22). Also, the respective 1.h.s. should more generally read

$$
-\frac{\partial \ln \mathcal{S}_{R}(\boldsymbol{x}, \boldsymbol{y})}{\partial \omega}=C_{R} \int_{0}^{L} \mathrm{~d} t \frac{\partial \Gamma_{\omega}(t, \boldsymbol{x}, \boldsymbol{y})}{\partial \omega} .
$$

After these replacements, the overall factor of $C_{R}$ cancels out and eq. (4.22) reduces to eq. (4.24) for $\Gamma_{\omega}(\boldsymbol{x}, \boldsymbol{y})$ for any value of $N_{c}$. Hence, as already mentioned in the main text, this equation is independent of the color representation $R$ of the dipole that we have started with.

Open Access. This article is distributed under the terms of the Creative Commons Attribution License (CC-BY 4.0), which permits any use, distribution and reproduction in any medium, provided the original author(s) and source are credited.

\section{References}

[1] Y. Mehtar-Tani, J.G. Milhano and K. Tywoniuk, Jet physics in heavy-ion collisions, Int. J. Mod. Phys. A 28 (2013) 1340013 [arXiv:1302.2579] [INSPIRE].

[2] A. Majumder and M. Van Leeuwen, The theory and phenomenology of perturbative QCD based jet quenching, Prog. Part. Nucl. Phys. A 66 (2011) 41 [arXiv:1002.2206] [InSPIRE].

[3] D. d'Enterria, Jet quenching, arXiv:0902.2011 [INSPIRE]. 
[4] J. Casalderrey-Solana and C.A. Salgado, Introductory lectures on jet quenching in heavy ion collisions, Acta Phys. Polon. B 38 (2007) 3731 [arXiv: 0712.3443] [INSPIRE].

[5] A. Kovner and U.A. Wiedemann, Gluon radiation and parton energy loss, hep-ph/0304151 [INSPIRE].

[6] P.B. Arnold and W. Xiao, High-energy jet quenching in weakly-coupled quark-gluon plasmas, Phys. Rev. D 78 (2008) 125008 [arXiv:0810.1026] [InSPIRE].

[7] S. Caron-Huot, $O(g)$ plasma effects in jet quenching, Phys. Rev. D 79 (2009) 065039 [arXiv:0811.1603] [INSPIRE].

[8] A. Majumder, Calculating the jet quenching parameter $\hat{q}$ in lattice gauge theory, Phys. Rev. C 87 (2013) 034905 [arXiv:1202.5295] [INSPIRE].

[9] T. Liou, A.H. Mueller and B. Wu, Radiative $p_{\perp}$-broadening of high-energy quarks and gluons in QCD matter, Nucl. Phys. A 916 (2013) 102 [arXiv:1304.7677] [INSPIRE].

[10] M. Laine and A. Rothkopf, Light-cone Wilson loop in classical lattice gauge theory, JHEP 07 (2013) 082 [arXiv:1304.4443] [INSPIRE].

[11] M. Panero, K. Rummukainen and A. Schäfer, A lattice study of the jet quenching parameter, Phys. Rev. Lett. 112 (2014) 162001 [arXiv:1307.5850] [INSPIRE].

[12] A.H. Mueller, Parton saturation: an overview, hep-ph/0111244 [INSPIRE].

[13] A.H. Mueller and S. Munier, $p_{\perp}$-broadening and production processes versus dipole/quadrupole amplitudes at next-to-leading order, Nucl. Phys. A 893 (2012) 43 [arXiv: 1206.1333] [INSPIRE].

[14] B. Wu, On $p_{T}$-broadening of high energy partons associated with the LPM effect in a finite-volume QCD medium, JHEP 10 (2011) 029 [arXiv:1102.0388] [INSPIRE].

[15] Y. Mehtar-Tani, Probabilistic picture of in-medium jet evolution, arXiv:1404.7448 [INSPIRE].

[16] J.-P. Blaizot and Y. Mehtar-Tani, Renormalization of the jet-quenching parameter, arXiv: 1403.2323 [INSPIRE].

[17] Y.L. Dokshitzer, V.A. Khoze, A.H. Mueller and S.I. Troian, Basics of perturbative QCD, Ed. Frontieres, Gif-sur-Yvette France (1991).

[18] Y.V. Kovchegov and E. Levin, Quantum chromodynamics at high energy, Cambridge University Press, Cambridge U.K. (2012).

[19] I. Balitsky, Operator expansion for high-energy scattering, Nucl. Phys. B 463 (1996) 99 [hep-ph/9509348] [INSPIRE].

[20] Y.V. Kovchegov, Small-x F(2) structure function of a nucleus including multiple Pomeron exchanges, Phys. Rev. D 60 (1999) 034008 [hep-ph/9901281] [INSPIRE].

[21] J. Jalilian-Marian, A. Kovner, A. Leonidov and H. Weigert, The BFKL equation from the Wilson renormalization group, Nucl. Phys. B 504 (1997) 415 [hep-ph/9701284] [INSPIRE].

[22] J. Jalilian-Marian, A. Kovner, A. Leonidov and H. Weigert, The Wilson renormalization group for low x physics: Towards the high density regime, Phys. Rev. D 59 (1998) 014014 [hep-ph/9706377] [INSPIRE].

[23] J. Jalilian-Marian, A. Kovner and H. Weigert, The Wilson renormalization group for low $x$ physics: gluon evolution at finite parton density, Phys. Rev. D 59 (1998) 014015 [hep-ph/9709432] [INSPIRE]. 
[24] A. Kovner, J.G. Milhano and H. Weigert, Relating different approaches to nonlinear QCD evolution at finite gluon density, Phys. Rev. D 62 (2000) 114005 [hep-ph/0004014] [INSPIRE].

[25] H. Weigert, Unitarity at small Bjorken x, Nucl. Phys. A 703 (2002) 823 [hep-ph/0004044] [INSPIRE].

[26] E. Iancu, A. Leonidov and L.D. McLerran, Nonlinear gluon evolution in the color glass condensate. 1, Nucl. Phys. A 692 (2001) 583 [hep-ph/0011241] [INSPIRE].

[27] E. Iancu, A. Leonidov and L.D. McLerran, The renormalization group equation for the color glass condensate, Phys. Lett. B 510 (2001) 133 [hep-ph/0102009] [INSPIRE].

[28] E. Iancu and L.D. McLerran, Saturation and universality in QCD at small x, Phys. Lett. B 510 (2001) 145 [hep-ph/0103032] [INSPIRE].

[29] E. Ferreiro, E. Iancu, A. Leonidov and L. McLerran, Nonlinear gluon evolution in the color glass condensate. 2, Nucl. Phys. A 703 (2002) 489 [hep-ph/0109115] [INSPIRE].

[30] L.V. Gribov, E.M. Levin and M.G. Ryskin, Semihard processes in QCD, Phys. Rept. 100 (1983) 1 [INSPIRE].

[31] E. Iancu, K. Itakura and L. McLerran, Geometric scaling above the saturation scale, Nucl. Phys. A 708 (2002) 327 [hep-ph/0203137] [INSPIRE].

[32] A.H. Mueller and D.N. Triantafyllopoulos, The energy dependence of the saturation momentum, Nucl. Phys. B 640 (2002) 331 [hep-ph/0205167] [INSPIRE].

[33] S. Munier and R.B. Peschanski, Geometric scaling as traveling waves, Phys. Rev. Lett. 91 (2003) 232001 [hep-ph/0309177] [INSPIRE].

[34] Z.-B. Kang, E. Wang, X.-N. Wang and H. Xing, Next-to-leading QCD factorization for semi-inclusive deep inelastic scattering at twist-4, Phys. Rev. Lett. 112 (2014) 102001 [arXiv:1310.6759] [INSPIRE].

[35] E. Iancu, A. Leonidov and L. McLerran, The color glass condensate: an introduction, hep-ph/0202270 [INSPIRE].

[36] F. Gelis, E. Iancu, J. Jalilian-Marian and R. Venugopalan, The color glass condensate, Ann. Rev. Nucl. Part. Sci. 60 (2010) 463 [arXiv:1002.0333] [InSPIRE].

[37] A. Kovner and U.A. Wiedemann, Eikonal evolution and gluon radiation, Phys. Rev. D 64 (2001) 114002 [hep-ph/0106240] [INSPIRE].

[38] E. Iancu, K. Itakura and L. McLerran, A gaussian effective theory for gluon saturation, Nucl. Phys. A 724 (2003) 181 [hep-ph/0212123] [INSPIRE].

[39] J.P. Blaizot, F. Gelis and R. Venugopalan, High-energy $p A$ collisions in the color glass condensate approach. 2. Quark production, Nucl. Phys. A 743 (2004) 57 [hep-ph/0402257] [INSPIRE].

[40] Y.V. Kovchegov, J. Kuokkanen, K. Rummukainen and H. Weigert, Subleading- $N_{c}$ corrections in non-linear small-x evolution, Nucl. Phys. A 823 (2009) 47 [arXiv:0812.3238] [INSPIRE].

[41] F. Dominguez, C. Marquet, B.-W. Xiao and F. Yuan, Universality of unintegrated gluon distributions at small x, Phys. Rev. D 83 (2011) 105005 [arXiv:1101.0715] [INSPIRE].

[42] E. Iancu and D.N. Triantafyllopoulos, Higher-point correlations from the JIMWLK evolution, JHEP 11 (2011) 105 [arXiv:1109.0302] [INSPIRE].

[43] E. Iancu and D.N. Triantafyllopoulos, JIMWLK evolution in the gaussian approximation, JHEP 04 (2012) 025 [arXiv: 1112.1104] [INSPIRE]. 
[44] A. Dumitru, J. Jalilian-Marian, T. Lappi, B. Schenke and R. Venugopalan, Renormalization group evolution of multi-gluon correlators in high energy QCD, Phys. Lett. B 706 (2011) 219 [arXiv: 1108.4764] [INSPIRE].

[45] J. Casalderrey-Solana and X.-N. Wang, Energy dependence of jet transport parameter and parton saturation in quark-gluon plasma, Phys. Rev. C 77 (2008) 024902 [arXiv:0705.1352] [INSPIRE].

[46] R. Baier, Y.L. Dokshitzer, A.H. Mueller, S. Peigne and D. Schiff, Radiative energy loss of high-energy quarks and gluons in a finite volume quark-gluon plasma, Nucl. Phys. B 483 (1997) 291 [hep-ph/9607355] [INSPIRE].

[47] R. Baier, Y.L. Dokshitzer, A.H. Mueller, S. Peigne and D. Schiff, Radiative energy loss and $p_{T}$ broadening of high-energy partons in nuclei, Nucl. Phys. B 484 (1997) 265 [hep-ph/9608322] [INSPIRE].

[48] B.G. Zakharov, Fully quantum treatment of the Landau-Pomeranchuk-Migdal effect in QED and QCD, JETP Lett. 63 (1996) 952 [hep-ph/9607440] [inSPIRE].

[49] B.G. Zakharov, Radiative energy loss of high-energy quarks in finite size nuclear matter and quark-gluon plasma, JETP Lett. 65 (1997) 615 [hep-ph/9704255] [INSPIRE].

[50] R. Baier, Y.L. Dokshitzer, A.H. Mueller and D. Schiff, Radiative energy loss of high-energy partons traversing an expanding QCD plasma, Phys. Rev. C 58 (1998) 1706 [hep-ph/9803473] [INSPIRE].

[51] R. Baier, Y.L. Dokshitzer, A.H. Mueller and D. Schiff, Medium induced radiative energy loss: equivalence between the BDMPS and Zakharov formalisms, Nucl. Phys. B 531 (1998) 403 [hep-ph/9804212] [INSPIRE].

[52] U.A. Wiedemann, Gluon radiation off hard quarks in a nuclear environment: opacity expansion, Nucl. Phys. B 588 (2000) 303 [hep-ph/0005129] [INSPIRE].

[53] U.A. Wiedemann, Jet quenching versus jet enhancement: a quantitative study of the BDMPS-Z gluon radiation spectrum, Nucl. Phys. A 690 (2001) 731 [hep-ph/0008241] [INSPIRE].

[54] P.B. Arnold, G.D. Moore and L.G. Yaffe, Photon emission from ultrarelativistic plasmas, JHEP 11 (2001) 057 [hep-ph/0109064] [INSPIRE].

[55] P.B. Arnold, G.D. Moore and L.G. Yaffe, Photon emission from quark gluon plasma: Complete leading order results, JHEP 12 (2001) 009 [hep-ph/0111107] [INSPIRE].

[56] P.B. Arnold, G.D. Moore and L.G. Yaffe, Photon and gluon emission in relativistic plasmas, JHEP 06 (2002) 030 [hep-ph/0204343] [INSPIRE].

[57] Z. Chen and A.H. Mueller, The Dipole picture of high-energy scattering, the BFKL equation and many gluon compound states, Nucl. Phys. B 451 (1995) 579 [INSPIRE].

[58] J.P. Blaizot, F. Gelis and R. Venugopalan, High-energy $p A$ collisions in the color glass condensate approach. 1. Gluon production and the Cronin effect, Nucl. Phys. A 743 (2004) 13 [hep-ph/0402256] [INSPIRE].

[59] A.H. Mueller, A simple derivation of the JIMWLK equation, Phys. Lett. B 523 (2001) 243 [hep-ph/0110169] [INSPIRE].

[60] S. Jeon, Color glass condensate in Schwinger-Keldysh QCD, Annals Phys. 340 (2014) 119 [arXiv: 1308.0263] [INSPIRE].

[61] S. Caron-Huot, When does the gluon reggeize?, arXiv:1309.6521 [INSPIRE].

[62] D. Binosi, A. Quadri and D.N. Triantafyllopoulos, High-energy QCD evolution from BRST symmetry, Eur. Phys. J. C 74 (2014) 2928 [arXiv:1402.4022] [INSPIRE]. 
[63] Y. Hatta, E. Iancu, K. Itakura and L. McLerran, Odderon in the color glass condensate, Nucl. Phys. A 760 (2005) 172 [hep-ph/0501171] [INSPIRE].

[64] L.N. Lipatov, Reggeization of the vector meson and the vacuum singularity in nonabelian gauge theories, Sov. J. Nucl. Phys. 23 (1976) 338 [InSPIRE].

[65] E.A. Kuraev, L.N. Lipatov and V.S. Fadin, The Pomeranchuk singularity in nonabelian gauge theories, Sov. Phys. JETP 45 (1977) 199 [INSPIRE].

[66] I.I. Balitsky and L.N. Lipatov, The Pomeranchuk singularity in quantum chromodynamics, Sov. J. Nucl. Phys. 28 (1978) 822 [INSPIRE].

[67] L.D. McLerran and R. Venugopalan, Computing quark and gluon distribution functions for very large nuclei, Phys. Rev. D 49 (1994) 2233 [hep-ph/9309289] [INSPIRE].

[68] L.D. McLerran and R. Venugopalan, Green's functions in the color field of a large nucleus, Phys. Rev. D 50 (1994) 2225 [hep-ph/9402335] [INSPIRE].

[69] P.B. Arnold, Simple formula for high-energy gluon bremsstrahlung in a finite, expanding medium, Phys. Rev. D 79 (2009) 065025 [arXiv:0808.2767] [INSPIRE].

[70] Y.V. Kovchegov and K. Tuchin, Inclusive gluon production in DIS at high parton density, Phys. Rev. D 65 (2002) 074026 [hep-ph/0111362] [INSPIRE].

[71] J.-P. Blaizot, F. Dominguez, E. Iancu and Y. Mehtar-Tani, Probabilistic picture for medium-induced jet evolution, JHEP 06 (2014) 075 [arXiv:1311.5823] [INSPIRE].

[72] Y. Hatta, E. Iancu and A.H. Mueller, Deep inelastic scattering off a $N=4$ SYM plasma at strong coupling, JHEP 01 (2008) 063 [arXiv:0710.5297] [INSPIRE].

[73] Y. Hatta, E. Iancu and A.H. Mueller, Jet evolution in the $N=4$ SYM plasma at strong coupling, JHEP 05 (2008) 037 [arXiv:0803.2481] [INSPIRE].

[74] F. Dominguez, C. Marquet, A.H. Mueller, B. Wu and B.-W. Xiao, Comparing energy loss and p-perpendicular-broadening in perturbative $Q C D$ with strong coupling $N=4 S Y M$ theory, Nucl. Phys. A 811 (2008) 197 [arXiv:0803.3234] [InSPIRE].

[75] Y. Hatta, E. Iancu and A.H. Mueller, Deep inelastic scattering at strong coupling from gauge/string duality: the saturation line, JHEP 01 (2008) 026 [arXiv:0710.2148] [INSPIRE].

[76] Y. Mehtar-Tani, Relating the description of gluon production in $p A$ collisions and parton energy loss in AA collisions, Phys. Rev. C 75 (2007) 034908 [hep-ph/0606236] [InSPIRE].

[77] J. Casalderrey-Solana and E. Iancu, Interference effects in medium-induced gluon radiation, JHEP 08 (2011) 015 [arXiv: 1105.1760] [INSPIRE].

[78] J.-P. Blaizot, F. Dominguez, E. Iancu and Y. Mehtar-Tani, Medium-induced gluon branching, JHEP 01 (2013) 143 [arXiv: 1209.4585] [INSPIRE].

[79] Y. Mehtar-Tani, C.A. Salgado and K. Tywoniuk, Anti-angular ordering of gluon radiation in QCD media, Phys. Rev. Lett. 106 (2011) 122002 [arXiv:1009.2965] [InSPIRE].

[80] Y. Mehtar-Tani, C.A. Salgado and K. Tywoniuk, Jets in QCD media: from color coherence to decoherence, Phys. Lett. B 707 (2012) 156 [arXiv:1102.4317] [InSPIRE].

[81] N. Armesto, H. Ma, Y. Mehtar-Tani, C.A. Salgado and K. Tywoniuk, Coherence effects and broadening in medium-induced QCD radiation off a massive q $\bar{q}$ antenna, JHEP 01 (2012) 109 [arXiv: 1110.4343] [INSPIRE].

[82] J.-P. Blaizot, E. Iancu and Y. Mehtar-Tani, Medium-induced QCD cascade: democratic branching and wave turbulence, Phys. Rev. Lett. 111 (2013) 052001 [arXiv:1301.6102] [INSPIRE]. 
[83] D.N. Triantafyllopoulos, The energy dependence of the saturation momentum from $R G$ improved BFKL evolution, Nucl. Phys. B 648 (2003) 293 [hep-ph/0209121] [INSPIRE].

[84] J.L. Albacete and Y.V. Kovchegov, Solving high energy evolution equation including running coupling corrections, Phys. Rev. D 75 (2007) 125021 [arXiv:0704.0612] [INSPIRE].

[85] I. Balitsky and G.A. Chirilli, Rapidity evolution of Wilson lines at the next-to-leading order, Phys. Rev. D 88 (2013) 111501 [arXiv:1309.7644] [INSPIRE].

[86] A. Kovner, M. Lublinsky and Y. Mulian, Jalilian-Marian, Iancu, McLerran, Weigert, Leonidov, Kovner evolution at next to leading order, Phys. Rev. D 89 (2014) 061704 [arXiv: 1310.0378] [INSPIRE].

[87] J.L. Albacete and C. Marquet, Gluon saturation and initial conditions for relativistic heavy ion collisions, Prog. Part. Nucl. Phys. 76 (2014) 1 [arXiv:1401.4866] [INSPIRE].

[88] S.S. Gubser and A. Karch, From gauge-string duality to strong interactions: a pedestrian's guide, Ann. Rev. Nucl. Part. Sci. 59 (2009) 145 [arXiv:0901.0935] [inSPIRE].

[89] J. Casalderrey-Solana, H. Liu, D. Mateos, K. Rajagopal and U.A. Wiedemann, Gauge/string duality, hot QCD and heavy ion collisions, arXiv:1101.0618 [INSPIRE].

[90] S.S. Gubser, D.R. Gulotta, S.S. Pufu and F.D. Rocha, Gluon energy loss in the gauge-string duality, JHEP 10 (2008) 052 [arXiv: 0803.1470] [inSPIRE].

[91] P.M. Chesler, K. Jensen, A. Karch and L.G. Yaffe, Light quark energy loss in strongly-coupled $N=4$ supersymmetric Yang-Mills plasma, Phys. Rev. D 79 (2009) 125015 [arXiv: 0810.1985] [INSPIRE].

[92] P. Arnold and D. Vaman, Jet quenching in hot strongly coupled gauge theories revisited: 3-point correlators with gauge-gravity duality, JHEP 10 (2010) 099 [arXiv:1008.4023] [INSPIRE].

[93] D. Binosi and L. Theussl, JaxoDraw: a graphical user interface for drawing Feynman diagrams, Comput. Phys. Commun. 161 (2004) 76 [hep-ph/0309015] [INSPIRE]. 